\title{
Continuous Wave Ring-Down Spectroscopy for Velocity Distribution Measurements in Plasma
}

\author{
Dustin W. McCarren
}

Follow this and additional works at: https://researchrepository.wvu.edu/etd

\section{Recommended Citation}

McCarren, Dustin W., "Continuous Wave Ring-Down Spectroscopy for Velocity Distribution Measurements in Plasma" (2015). Graduate Theses, Dissertations, and Problem Reports. 6188.

https://researchrepository.wvu.edu/etd/6188

This Dissertation is protected by copyright and/or related rights. It has been brought to you by the The Research Repository @ WVU with permission from the rights-holder(s). You are free to use this Dissertation in any way that is permitted by the copyright and related rights legislation that applies to your use. For other uses you must obtain permission from the rights-holder(s) directly, unless additional rights are indicated by a Creative Commons license in the record and/ or on the work itself. This Dissertation has been accepted for inclusion in WVU Graduate Theses, Dissertations, and Problem Reports collection by an authorized administrator of The Research Repository @ WVU.

For more information, please contact researchrepository@mail.wvu.edu. 


\title{
Continuous Wave Ring-Down Spectroscopy for Velocity Distribution Measurements in Plasma
}

\author{
Dustin W. MCCARren \\ Dissertation submitted to the College of Arts and Sciences \\ at West Virginia University \\ in partial fulfillment of the requirements \\ for the degree of \\ Doctor of Philosophy \\ in \\ Physics \\ Earl E. Scime, Ph.D. Chair \\ Alan D. Bristow, Ph.D. \\ Paul A. Cassak, Ph.D. \\ Fabien Goulay, Ph.D. \\ Amy M. Keesee, Ph.D. \\ Department of Physics and Astronomy \\ Morgantown, West Virginia \\ 2014 \\ Keywords: spectroscopy, diagnostic \\ Copyright 2014 Dustin W. McCarren
}




\section{ABSTRACT \\ Continuous Wave Ring-Down Spectroscopy for Velocity Distribution \\ Measurements in Plasma}

\section{Dustin W. McCarren}

Cavity Ring-Down Spectroscopy CRDS is a proven, ultra-sensitive, cavity enhanced absorption spectroscopy technique. When combined with a continuous wavelength $(\mathrm{CW})$ diode laser that has a sufficiently narrow line width, the Doppler broadened absorption line, i.e., the velocity distribution functions (VDFs) of the absorbing species, can be measured. Measurements of VDFs can be made using established techniques such as laser induced fluorescence (LIF). However, LIF suffers from the requirement that the initial state of the LIF sequence have a substantial density and that the excitation scheme fluoresces at an easily detectable wavelength. This usually limits LIF to ions and atoms with large metastable state densities for the given plasma conditions. CW-CRDS is considerably more sensitive than LIF and can potentially be applied to much lower density populations of ion and atom states. Also, as a direct absorption technique, CW-CRDS measurements only need to be concerned with the species absorption wavelength and provide an absolute measure of the line integrated initial state density. Presented in this work are measurements of argon ion and neutral VDFs in a helicon plasma using CW-CRDS and LIF. 


\section{Acknowledgement}

This is easily the most challenging portion of this entire work and the one I am sure that will be the most lacking in thoroughness, despite my best efforts.

I owe thanks to:

Dr. Earl Scime for giving me a chance to prove myself and for all that he taught me.

The fantastic Physics Department faculty and staff especially, Dr. Arthur Weldon, Dr. Paul Cassak, Sherry Puskar, Devon Cleland, Carl Weber, Doug Mathess and J.R. Raber.

My fellow WVU graduate students and the few undergrads I have had the pleasure to work with.

Thank you Dr. Colin Komar, Dr. Matthew E. Galante, Drew Elliot, John McKee, Greg

Lusk, Dr. Saikat Chakraborty Thakur and Dr. Jerry Carr Jr. for being friends as well as colleagues.

My family who always supported me.

My Mother, who made sure I never did without.

My sister who made sure I retained my sanity when confounded.

My Grandparents who always had time for me and let a curious boy tinker in their garage. 
If you're going through Hell, keep going. -Winston Churchill 


\section{Contents}

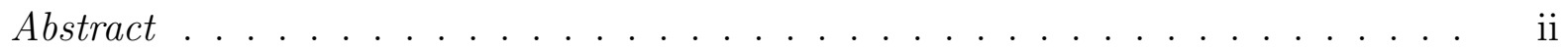

Acknowledgements ..................... iii

Words of Wisdom. . . . . . . . . . . . . . . . . . $\mathrm{v}$

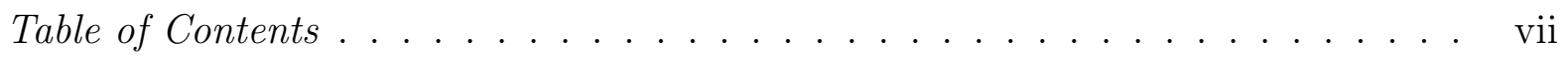

List of Figures . . . . . . . . . . . . . . . . . $\mathrm{x}$

1 Introduction $\quad 1$

$\begin{array}{llr}2 & \text { Helicons } & 7\end{array}$

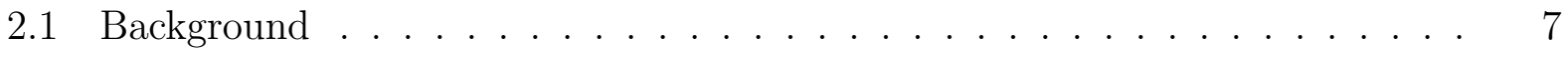

2.2 Experimental Setup . . . . . . . . . . . . . . . . . . 8

$2.2 .1 \quad$ Chamber. . . . . . . . . . . . . . . . . . . . . . 9

2.2 .2 Vacuum System . . . . . . . . . . . . . . . . . . . . . 9

$2.2 .3 \quad$ Magnetic Field . . . . . . . . . . . . . . . . . . . . . . . . . 9

2.2.4 RF Antenna and Matching Network . . . . . . . . . . . . . . . 11

$2.2 .5 \quad$ Plasma Parameters . . . . . . . . . . . . . . . . . . . . 12

3 Laser Induced Fluorescence $\quad 15$

3.1 Theory . . . . . . . . . . . . . . . . . . . . . 16 
3.2 Experimental Apparatus . . . . . . . . . . . . . . . . 18

3.3 Data Analysis . . . . . . . . . . . . . . . . . . . . 19

3.4 Measurements . . . . . . . . . . . . . . . . . . 20

4 CW-CRDS $\quad 23$

4.1 Absorption spectroscopy ............................ 24

4.2 Pulsed Cavity Ring-Down Spectroscopy . . . . . . . . . . . . . . . . 25

4.3 Continuous Wave Cavity Ring-down Spectroscopy . . . . . . . . . . . . . 29

4.3 .1 Free Spectral Range . . . . . . . . . . . . . . . . . 30

4.3.2 The Cavity Finesse . . . . . . . . . . . . . . . . . . . . 31

4.3 .3 Cavity Stability . . . . . . . . . . . . . . . 31

4.3.4 Cavity Mode Structure . . . . . . . . . . . . . . . 32

4.3.5 The Cavity Spot Size . . . . . . . . . . . . . . . 33

4.3.6 Mode-Matching . . . . . . . . . . . . . . . 34

4.4 CW-CRDS Experimental Apparatus, MARK-I . . . . . . . . . . 36

4.5 Initial CW-CRDS measurements . . . . . . . . . . . . . . . 39

4.6 Zeroth Order Error Propagation Analysis . . . . . . . . . . . . . . . . 41

4.6.1 Sub-Optimal Mode Matching . . . . . . . . . . . . . . 41

4.6.2 Injection Beam Extinction . . . . . . . . . . . . . . . 42

4.6.3 Particle Depositions on Mirrors . . . . . . . . . . . . . . 44

4.6.4 Mechanical Instability . . . . . . . . . . . . . . . 45

4.7 CW-CRDS Experimental Apparatus, MARK-II . . . . . . . . . . . 47

4.8 CW-CRDS MARK-II Data Analysis . . . . . . . . . . . . . 50

5 CW-CRDS and LIF $\quad 55$ 


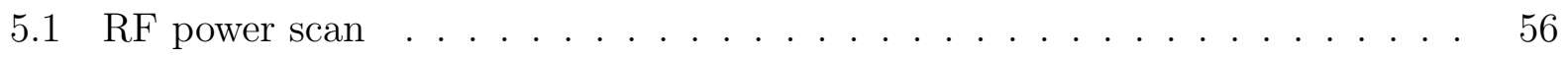

5.2 Magnetic Field Scan . . . . . . . . . . . . . . . . . . 61

5.3 Ar I and Ar II measurements . . . . . . . . . . . . . . . . 66

5.3.1 Ar II measurements . . . . . . . . . . . . . . . . 67

5.3.2 Ar I measurements . . . . . . . . . . . . . . . . . 73

5.4 Discussion . . . . . . . . . . . . . . . . . . 77

6 Conclusion $\quad 79$

References .............................. 82 


\section{List of Figures}

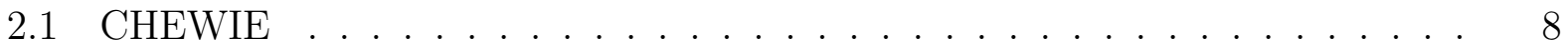

2.2 CHEWIE source rigion B-field . . . . . . . . . . . . . . . . . 10

2.3 CHEWIE expansion chamber B-field . . . . . . . . . . . . . . 11

2.4 CHEWIE antenna . . . . . . . . . . . . . . . . . . . . . . . 12

2.5 Matching network circuit . . . . . . . . . . . . . . . . . . . . . . . 12

3.1 Argon LIF excitation schemes for (a) Ar II and (b) Ar I. . . . . . . . . 17

$3.2 \quad$ LIF Experimental Setup . . . . . . . . . . . . . . . . . . . . . . . 19

3.3 Example lif measurement . . . . . . . . . . . . . . . . . . . . . . 20

3.4 LIF measurements of (a) Ar II and (b) Ar I at $P=5.4$ mTorr, $R F=300 \mathrm{~W}$, $B=860 \mathrm{G}$ and Mass flow $=5 \mathrm{SCCM} \ldots \ldots \ldots \ldots . \ldots \ldots$

3.5 LIF measurements of (a) Ar II and (b) Ar I at $P=5.6$ mTorr, $R F=300 \mathrm{~W}$, $B=800 \mathrm{G}$ and Mass flow $=5 \mathrm{SCCM} \ldots \ldots \ldots \ldots . \ldots \ldots$

3.6 LIF measurements of (a) Ar II and (b) Ar I at $P=7.3 \mathrm{mTorr}, R F=300 \mathrm{~W}$, $B=750 \mathrm{G}$ and Mass flow $=5 \mathrm{SCCM} \ldots \ldots \ldots \ldots \ldots \ldots$

4.1 Absorption Spectroscopy Experimental Setup Sketch _. . . . . . . . . . . 24

4.2 P-CRDS Experimental Setup Sketch _ . . . . . . . . . . . . 25 
4.3 Light "bouncing" in a ring-down cavity . . . . . . . . . . . . . . 26

4.4 Pulsed broad band laser . . . . . . . . . . . . . . . . . . 29

4.5 CW-CRDS Experimental Setup Sketch . . . . . . . . . . . . . 29

4.6 Cavity Mode Spacing . . . . . . . . . . . . . . . . . . . . 32

4.7 TEM modes . . . . . . . . . . . . . . . . . . . . 33

4.8 Cavity Spot Size . . . . . . . . . . . . . . . . . . . 34

4.9 Mode Matching . . . . . . . . . . . . . . . . . . . 35

$4.10 \mathrm{CW}-\mathrm{CRDS}$ MRKI . . . . . . . . . . . . . . . 37

4.11 CW-CRDS MARK-I mounts . . . . . . . . . . . . . . . 38

4.12 CW-CRDS MRKI tracking circuit . . . . . . . . . . . . . . . 39

4.13 CW-CRDS MARK-I proof-of-principal measurement . . . . . . . . . . 40

4.14 Effects of sub-optimal mode matching . . . . . . . . . . . . . . . 42

4.15 Excitation of higher order modes during a ring-down . . . . . . . . . . 43

4.16 Effects of injection beam excitation . . . . . . . . . . . . . . 44

4.17 CW-CRDS mirror degradation . . . . . . . . . . . . . . 45

4.18 Mechanical instability effects . . . . . . . . . . . . . . 46

4.19 MRKII cw-crds experimental setup . . . . . . . . . . . . . . . . 48

4.20 MRKII cw-crds mirror mounts . . . . . . . . . . . . . . . . . 49

4.21 Single ring-down measurement f . . . . . . . . . . . . . . . 51

4.22 Distribution of ring-down measurements . . . . . . . . . . . . . 52

4.23 Distribution of line integrated absorption . . . . . . . . . . . 53

5.1 Argon ion (a) LIF and (b) CW-CRDS VDFs measured at $r f=300 \mathrm{~W}, B=750$ G, $P=8.3$ mTorr, mass flow $=6$ SCCM. . . . . . . . . . . . . . . 57 
5.2 Argon ion (a) LIF and (b) CW-CRDS VDFs measured at $r f=400 \mathrm{~W}, B=750$ G, $P=6.2$ mTorr, mass flow $=6$ SCCM. . . . . . . . . . . . . . 57

5.3 Argon ion (a) LIF and (b) CW-CRDS VDFs measured at $r f=500 \mathrm{~W}, B=750$ G, $P=7$ mTorr, mass flow $=6$ SCCM. . . . . . . . . . . . . . . 58

5.4 Argon ion (a) LIF and (b) CW-CRDS VDFs measured at $r f=600 \mathrm{~W}, B=750$ G, $P=6.2$ mTorr, mass flow $=6$ SCCM. . . . . . . . . . . . . . . 58

5.5 Argon ion (a) LIF and (b) CW-CRDS VDFs measured at $r f=700 \mathrm{~W}, B=750$ G, $P=5.1$ mTorr, mass flow=6 SCCM. . . . . . . . . . . . . . . 59

5.6 RF power scan temperatures . . . . . . . . . . . . . . . 60

5.7 RF power scan densities . . . . . . . . . . . . . . . . . . 61

5.8 Argon (a) LIF and (b) CW-CRDS VDFs measured at $r f=500 \mathrm{~W}, B=630 \mathrm{G}$, $P=8.4$ mTorr, mass flow $=6$ SCCM. . . . . . . . . . . . . . . . . . . 62

5.9 Argon (a) LIF and (b) CW-CRDS VDFs measured at $r f=500 \mathrm{~W}, B=690 \mathrm{G}$, $P=6.4$ mTorr, mass flow $=6$ SCCM. . . . . . . . . . . . . . . . . . .

5.10 Argon (a) LIF and (b) CW-CRDS VDFs measured at $r f=500 \mathrm{~W}, B=750 \mathrm{G}$, $P=7$ mTorr, mass flow $=6$ SCCM.

5.11 Argon (a) LIF and (b) CW-CRDS VDFs measured at $r f=500 \mathrm{~W}, B=800 \mathrm{G}$, $P=4.6$ mTorr, mass flow $=6$ SCCM. . . . . . . . . . . . . . . . .

5.12 Argon (a) LIF and (b) CW-CRDS VDFs measured at $r f=500 \mathrm{~W}, B=860 \mathrm{G}$, $P=3.8$ mTorr, mass flow $=6$ SCCM. . . . . . . . . . . . . . . . 64

5.13 B-Field scan temperatures for LIF and cw-crds . . . . . . . . . . . . . 65

5.14 B-Field scan densities . . . . . . . . . . . . . . . . . . . . . . 66

5.15 Argon ion (a) LIF and (b) CW-CRDS VDFs measured at $r f=300 \mathrm{~W}, B=630$ $\mathrm{G}, P=12.5$ mTorr mass flow $=5$ SCCM. . . . . . . . . . . . . . 67 
5.16 Argon ion (a) LIF and (b) CW-CRDS VDFs measured at $r f=300 \mathrm{~W}, B=690$ $\mathrm{G}, \mathrm{P}=9.8$ mTorr, mass flow $=5$ SCCM. . . . . . . . . . . . . . . 68

5.17 Argon ion (a) LIF and (b) CW-CRDS VDFs measured at $r f=300 \mathrm{~W}, B=750$ G, $P=7.8$ mTorr, mass flow $=5$ SCCM. . . . . . . . . . . . . . 68

5.18 Argon ion (a) LIF and (b) CW-CRDS VDFs measured at $r f=300 \mathrm{~W}, B=800$ G, $P=6.8$ mTorr, mass flow $=5$ SCCM. . . . . . . . . . . . . . . 69

5.19 Argon ion (a) LIF and (b) CW-CRDS VDFs measured at $r f=300 \mathrm{~W}, B=860$ G, $P=6.4$ mTorr, mass flow $=5$ SCCM. . . . . . . . . . . . . . . 69

5.20 Ar II B-Field scan temperatures . . . . . . . . . . . . . . . 71

5.21 Ar II B-Field scan densities . . . . . . . . . . . . . . . . . 72

5.22 Argon neutral (a) LIF and (b) CW-CRDS VDFs measured at $r f=300 \mathrm{~W}$, $B=860 \mathrm{G}, P=6.3$ mTorr, mass flow $=5$ SCCM. . . . . . . . . . . . 73

5.23 Argon neutral (a) LIF and (b) CW-CRDS VDFs measured at $r f=300 \mathrm{~W}$, $B=800 \mathrm{G}, P=6.8$ mTorr, mass flow $=5$ SCCM. . . . . . . . . . . . . 73

5.24 Argon neutral (a) LIF and (b) CW-CRDS VDFs measured at $r f=300 \mathrm{~W}$, $B=750 \mathrm{G}, P=7.6$ mTorr, mass flow $=5$ SCCM. . . . . . . . . . . . . 74

5.25 B-Field scan densities . . . . . . . . . . . . . . . . . . 74

5.26 B-Field scan densities . . . . . . . . . . . . . . . . . 75

5.27 Ar I B-Field power scan temperatures . . . . . . . . . . . . . . 76

5.28 Ar I B-Field power scan densities . . . . . . . . . . . . . . . . 76

5.29 Argon neutral CW-CRDS VDF made at 630 G . . . . . . . . . . . 78 


\section{Chapter 1}

\section{Introduction}

To conceptualize and quantify a variety of plasma physics phenomena, we need to understand the dynamics of the particles that constitute the plasma. It is impractical to attempt to measure and analyze every individual particle in a plasma where every cubic centimeter can consist of an inumerable number of particles. However, measurements of particle distributions provide insight into important plasma characteristics. By analyzing velocity distribution functions (VDFs) we are able to calculate plasma densities, temperatures, flows and other features.

A host of diagnostics and measurement techniques have been developed to measure VDFs, but they can all be placed in one of two categories, perturbative and non-perturbative. Perturbative measurements are made by immersing some material in the plasma, a probe, near the region of interest. Any material placed in a plasma will effectively perturb the characteristics in the region of interest. Optical diagnostics can be used to perform nonperturbative in situ measurements of VDFs in plasmas.

Laser induced fluorescence (LIF), an established optical diagnostic, can be used to obtain spatially resolved temperatures and flow speeds by measuring VDFs in plasmas. Despite 
LIF's versatility and sensitivity, the technique has limitations. To perform LIF measurements of a specie's natural transition lines, the initial state of the sequence must have a substantial density and the radiative decay of the excited state must fluoresce at an easily detectable wavelength. These necessities inherent to LIF have impaired our ability to resolve the VDFs of several species of interest.

For example, helium-based plasmas are of widespread scientific and industrial interest. However, helium ions are notoriously difficult to fluoresce. Transitions from the helium ion ground state are in the soft x-ray wavelength range and transitions from the metastable $2 \mathrm{~S}$ state are all in the ultraviolet or shorter region of the electromagnetic spectrum. Attempts at WVU to perform LIF on helium ions using the infrared $n=5$ to $n=6$ transition at $1012 \mathrm{~nm}$ have been unsuccessful. ${ }^{1}$ Due to the degeneracy of the excited state, even if the initial excitation were successful, nearly all of the fluorescent emission would be in the ultraviolet or soft x-ray regime. Very long path length absorption spectroscopy measurements at $1012 \mathrm{~nm}$ hinted at the existence of a sufficient population of absorbing species, but fluctuations in the probing laser intensity resulted in very poor signal-to-noise and irreproducible results. ${ }^{1}$

LIF measurements on the Ar I transition line at $667 \mathrm{~nm}$ performed by Keesee et at. ${ }^{2,3}$ also proved challenging, but for reasons different than the problems faced by measurements of the He II line. The Ar I excitation scheme's initial state is populated by excitations from the ground state and through electron impact transfers from nearby metastable states. Because of the scheme's dependence on collisional mechanisms to populate the initial state, LIF measurements of Ar I VDFs were unresolvable at low, less than 10 mTorr, operating pressures.

In this work I discuss the development of a continuous wave cavity ring-down spectroscopy (CW-CRDS), an ultra-sensitive, cavity enhanced absorption spectroscopy technique, to per- 
form VDF measurements unobtainable with laser induced fluorescence. CRDS is a relatively new laser based diagnostic technique. The use of the time constant of decaying light, the cavity ring-down time, in an optical cavity consisting of two plano-concave mirrors was first used by Anderson et al. ${ }^{4}$ in 1984 as a method of accurately calculating the reflectivity of dielectric coatings. The first cavity ring-down spectroscopic measurements were made by OKeefe and Deacon in $1988 .^{5}$ In their experiment, CRDS was used to measure the absorption spectrum of oxygen molecules in the optical cavity with a nitrogen-gas-pumped pulsed dye laser emitting at $690 \mathrm{~nm}$. The authors were able to obtain a minimum detectable absorption coefficient on the order of $10^{-6} \mathrm{~cm}^{-1}$ using this pulsed cavity ring-down spectroscopy (P-CRDS) diagnostic. ${ }^{5}$ Because of the relative simplicity of the experimental design and high levels of sensitivity obtainable by P-CRDS, it quickly became popular among spectroscopists in a variety of fields.

The use of a pulsed laser made P-CRDS readily achievable experimentally, but imposed limitations on the sensitivity of the cavity ring-down technique. The physics of P-CRDS was investigated by Hodges et al. at the National Institute of Standards and Technologies (NIST) in the mid to late 1990s. ${ }^{6,7}$ They showed that the linewidth of the pulsed laser source and the excitations of multiple modes in the ring-down cavity affect the maximum obtainable sensitivity. The authors suggested that single mode, narrow linewidth lasers should be used to maximize the potential of the CRDS technique.

The use of narrow linewidth lasers to perform CRDS measurements required a more intricate experimental setup than what was needed for P-CRDS. The first measurements using what is called continuous wave cavity ring-down spectroscopy (CW-CRDS) were made by Romanini, et al. in $1997 .{ }^{8}$ In their experiment, $570 \mathrm{~nm}$ light from a Coherent CR699 single frequency ring dye laser was sent through an acousto-optic modulator (AOM) 
and mode matched into a $50 \mathrm{~cm}$ long ring-down cavity. The cavity length was modulated by mounting one of the mirrors on piezo-electric transducer (PZT) and when a resonance condition was met, a tracking circuit used in conjunction with the AOM extinguished the injection beam. After the light was extinguished, the ring-down time of the light in the cavity was recorded. Using the CW-CRDS diagnostic technique, they were able to obtain a minimum detectable absorption coefficient on the order of $10^{-9} \mathrm{~cm}^{-1}$. $^{8}$

The use of CRDS for measuring properties of or associated with plasma devices is an active area of research. At Colorado State University, a CW-CRDS diagnostic technique is being developed. ${ }^{9}$ The non-ideal environment of a Hall thruster test chamber and the proximity of the potentially damaging sputtered particulates being measured provide many experimental challenges for their CW-CRDS diagnostic. At Mississippi State University, CRDS is being used to study atmospheric microwave plasma jets ${ }^{10}$. The research group at Missouri State has also designed a highly portable spectrometer that utilizes CRDS. ${ }^{11}$ As the CRDS diagnostic technique matures, more researchers are beginning to implement the ultra-sensitive technique in plasma physics experiments.

The purpose of this dissertation is to discuss the development of a CW-CRDS diagnostic and demonstrate its ability to perform VDF measurements unobtainable with laser induced fluorescence. A brief description of the CHEWIE plasma system used in these measurements is described in Chapter 2. In Chapter 3 I briefly review the theory of LIF and discuss Ar I and Ar II VDF measurements made at plasma parameters where Ar I VDFs become unmeasurable by LIF. Chapter 4 contains a discussion of the proof-of-principle measurement of AR II using the CW-CRDS technique performed by Chakborty Thakur ${ }^{12}$ with the initial, MARK-I, CW-CRDS apparatus. Unfortunately, the proof-of-principle measurement was plagued by poor signal-to-noise and yielded ion velocity distribution function (IVDF) line widths that 
disagreed sharply with those determined by LIF measurements. The remainder of Chapter 4 describes the improvements made to the MARK-I apparatus and the initial measurements with the improved system. In Chapter 5 I present comparison measurements of Ar II and Ar I VDFs obtained with LIF and CW-CRDS to validate the MARK-II apparatus and show the increased sensitivity of the CW-CRDS technique. 


\section{Chapter 2}

\section{Helicons}

\section{$2.1 \quad$ Background}

Helicon waves were first studied in solid state ${ }^{13}$ and gaseous plasmas ${ }^{14}$ in the 1960 s. The helicon plasma devices we know today were pioneered by Boswell ${ }^{15}$ and Chen. ${ }^{16} \mathrm{~A}$ detailed accounting of the development of helicons is given by Kline. ${ }^{17}$ Helicon plasma discharges are capable of producing plasma densities of $10^{13} \mathrm{~cm}^{-3}$ with only 100s of Watts of rf power and magnetic field strengths of 100s of Gauss. Due to their efficient, high density and low temperature nature, helicon plasmas are ideal for a myriad of applications. Current applications range from plasma propulsion for interplanetary travel to plasma processing during computer chip manufacturing.

The mechanism responsible for this efficient, high density ion production remains elusive despite years of studies. Investigators have proposed several theories including collisional processes, resonant coupling at the lower hybrid frequency and Landau damping, but no single theory is consistent with the full range of experimental observations. The neutral pressure, rf antenna design and magnetic field all affect the density profiles of helicon plasmas. 


\subsection{Experimental Setup}

The data presented in this work were obtained in the Compact Helicon for Waves and Instabilities Experiment (CHEWIE). As the name implies, CHEWIE is a small, compact helicon source that was originally designed to conduct plasma processing relevant research. ${ }^{18}$ In recent years, CHEWIE has undergone several modifications to make it better suited to act as a diagnostic test bed. In the rest of this section, the current CHEWIE plasma source is described. Thorough descriptions of the previous incarnation of CHEWIE are available in the literature. ${ }^{1920}$



Figure 2.1: Three-dimensional rendering of the CHEWIE plasma device. Pictured here are the two water cooled electromagnets, the $m=1 \mathrm{rf}$ saddle antenna, the plasma source tube and the plasma expansion chamber. 


\subsubsection{Chamber}

The CHEWIE plasma chamber consists of several components. The source tube, a $61 \mathrm{~cm}$ long, $5 \mathrm{~cm}$ inner diameter Pyrex ${ }^{\mathrm{TM}}$ tube is where plasma production occurs. One end of the source tube terminates in a $6 "$ stainless steel flange capped with a $2 \frac{3}{4}$ " window. The other end is connected to a $20 \mathrm{~cm}$ long, $15 \mathrm{~cm}$ diameter stainless steel chamber custom made for this experiment by Kurt J. Lesker ${ }^{\mathrm{TM}}$. The expansion chamber has an array of nine $2 \frac{3}{4}$ " Conflat $^{\mathrm{TM}}$ ports at two different axial locations, six near the source tube with three more a little further, axially, away from the source tube (Figure 2.1). Two of the six upper ports are reserved for pressure gauges, leaving a total of seven ports for plasma diagnostics.

\subsubsection{Vacuum System}

High vacuum conditions, with a base pressure of $10^{-7}$ Torr, are maintained by a Pfeiffer Vacuum TSH 521 turbomolecular drag pump backed with a diaphragm pump. The pumping system is coupled to the chamber by bellows to minimize vibrations, and a pneumatic gate valve. The pneumatic gate valve is interlocked with a KJL 4500 Ion Gauge Controller. In the event that the chamber is exposed to atmospheric pressures, the gate valve closes and isolates the pumping system. A MKS PR4000 power supply and MKS 1179A mass flow controller are used to control the rate of gas feed, typically 0.1-100 SCCM. Discharge operating pressures are 1-50 mTorr, measured with a species independent MKS 179 Type A Baratron gauge.

\subsubsection{Magnetic Field}

Two new water cooled aluminum electromagnets, constructed at West Virginia University, provide steady-state axial magnetic fields of up to 1200 G. The magnets are each made of 
seven sets of $\frac{1}{2}$ " aluminum square tubing wound into seven two-coil "pancakes" of two layers, giving 140 turns per magnet. Each magnet is $21.6 \mathrm{~cm}$ in height, with a $21.6 \mathrm{~cm}$ inner and $75 \mathrm{~cm}$ outer radius respectively. An Ametek Sorensen power supply provides current for the magnets. Maximum current is $395 \mathrm{~A}$ at $25 \mathrm{~V}$. A plot of the measured magnetic field strength versus applied current in the source region is shown in Figure 2.2. A plot of the magnetic field strength in the expansion chamber, the region of interest for this work, is shown in Figure 2.3. The $\sim 35 \%$ difference in magnitude agrees with previous measurements. ${ }^{21}$

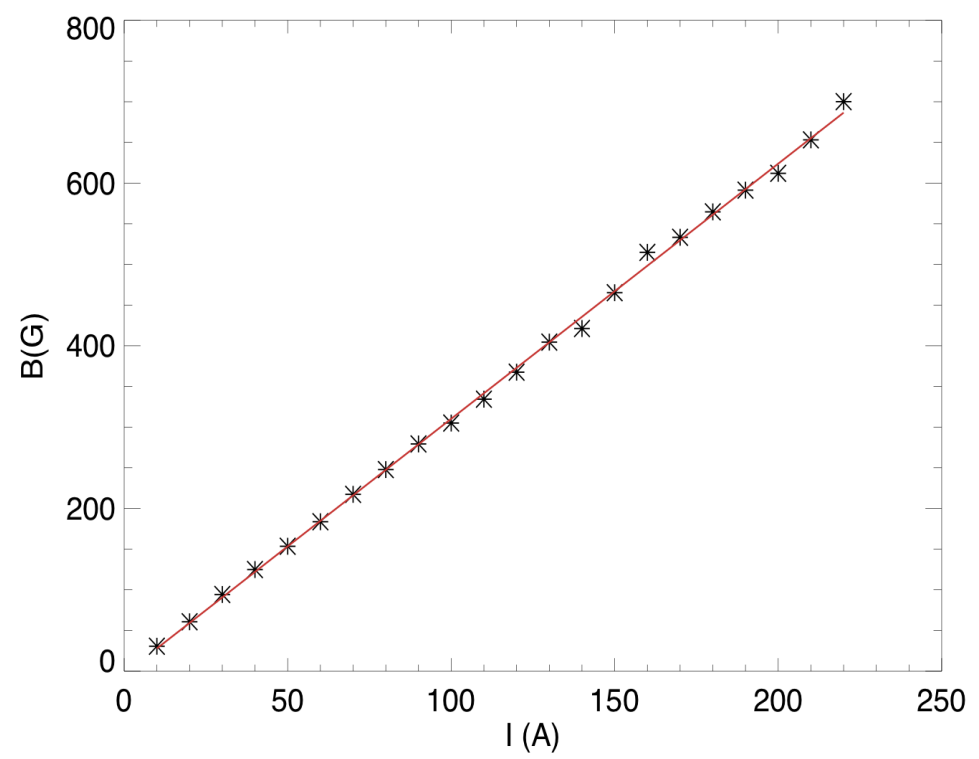

Figure 2.2: Measured magnetic field versus applied current in source region. Slope of fit line is 3.14 G/A. ${ }^{21}$ 


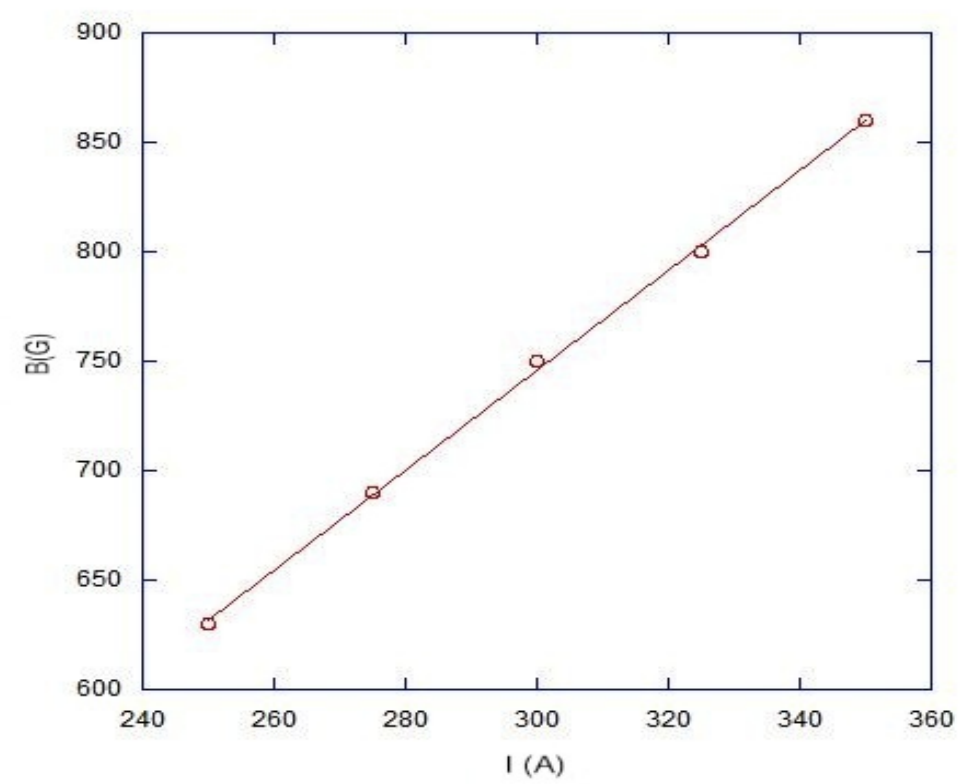

Figure 2.3: Measured magnetic field versus applied current in the expansion chamber region. Slope of fit line is $2.28 \mathrm{G} / \mathrm{A}$.

\subsubsection{RF Antenna and Matching Network}

The rf power supply for these experiments was a Dressler Cesar Model 1350 rf generator, which provides up to $5 \mathrm{~kW}$ of power at $13.56 \mathrm{MHz}$. An $18 \mathrm{~cm}, m=1$ right hand helical antenna placed around the source tube couples the rf power into the plasma (Figure 2.4). A two capacitor $\pi$ matching circuit is used to match the impedance of the plasma to the 50 ohm output impedance of the Dressler rf generator (Figure 2.5). Both capacitors are Jennings high-voltage vacuum capacitors with tuning ranges of 20-2000 pF. One capacitor, the tuning capacitor, is placed in series with the antenna and the other, the load capacitor, is placed parallel to the tuning capacitor/antennal branch of the circuit. A discussion of two 
capacitor impedance matching circuits can be found in Chen's report. ${ }^{22}$

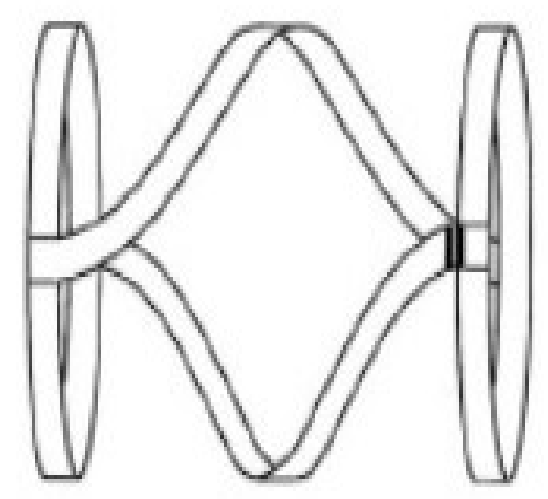

Figure 2.4: A sketch of an $m=1$ right hand helical antenna. ${ }^{23}$

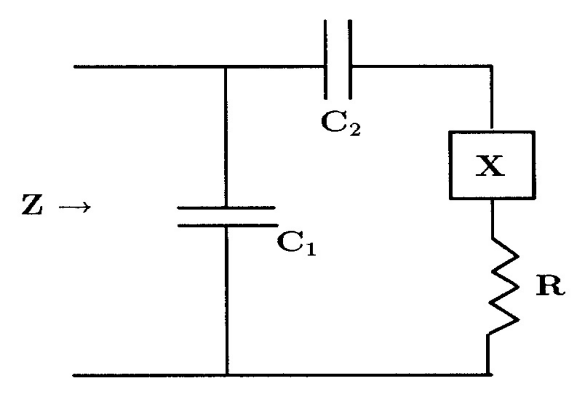

Standard Circuit

Figure 2.5: Circuit diagram of a $\pi$ matching network. $C 1$ is the load capacitor and $C 2$ is the tuning capacitor. $R$ and $X$ represent the real and imaginary parts of the resistance created by the antenna and plasma combination. ${ }^{22}$

\subsubsection{Plasma Parameters}

Bellow are the typical plasma parameters for CHEWIE for the experiments described in this work. 
Table 2.1: Representative operating parameters of CHEWIE

\begin{tabular}{|c|c|}
\hline Parameter [Symbol] (units) & $\mathrm{Ar}$ \\
\hline Magnetic Field $[B]($ Gauss $)$ & $600-1200$ \\
\hline Pressure $[p](\mathrm{mTorr})$ & 20 \\
\hline Electron Temperature $\left[T_{e}\right](\mathrm{eV})$ & $3-8$ \\
\hline Ion Temperature $\left[T_{i}\right](\mathrm{eV})$ & .16 \\
\hline Density $[n]\left(\mathrm{x} 10^{10} \mathrm{~cm}^{-3}\right)$ & $1-10$ \\
\hline Debye Length $\left[\lambda_{D}\right](\mathrm{cm})$ & $7.8 \times 10^{-3}$ \\
\hline Plasma Frequency $\left[\omega_{p e}\right]\left(10^{10} \mathrm{rad} / \mathrm{s}\right)$ & 1.26 \\
\hline Ion Cyclotron Frequency $\left[\omega_{c i}\right]\left(10^{5} \mathrm{rad} / \mathrm{s}\right)$ & 2.2 \\
\hline Electron Cyclotron Frequency $\left[\omega_{c e}\right]\left(10^{9} \mathrm{rad} / \mathrm{s}\right)$ & 16 \\
\hline Ion Larmor Radius $\left[r_{i}\right](\mathrm{cm})$ & $4 \times 10^{-1}$ \\
\hline Electron Larmor Radius $\left[r_{e}\right](\mathrm{cm})$ & $8 \times 10^{-3}$ \\
\hline
\end{tabular}




\section{Chapter 3}

\section{Laser Induced Fluorescence}

Since the invention of the laser, a variety of laser based diagnostics have been developed. Laser induced fluorescence (LIF) was first demonstrated by Yardley and Moore in $1966 .{ }^{24}$ In their experiments, they excited $\mathrm{CO}_{2}$ molecules using an infrared laser and measured the fluorescence radiated by the excited molecules as they decayed to a lower energy state. The first plasma physics application of LIF was performed by Stern and Johnson nearly a decade later in $1975 .{ }^{25}$ In their experiments, the emission line shape of excited argon ions was measured using a spectrometer; allowing them to resolve the ion velocity distribution function (IVDF). Hill et $a l^{26}$ were able to increase the sensitivity and resolution of temperatures and plasma flow speed measurements by LIF by two orders of magnitude by using a narrow band laser to measure the absorption line shape to resolve the IVDF. A brief overview of LIF, the experimental setup, and measurements pertinent to this work are presented in this chapter. 


\subsection{Theory}

Laser induced fluorescence is achieved by using a laser tuned to the natural absorption line of a molecule, atom, or ion in what is typically a ground or metastable state. The absorbing species is then excited to a higher radiative state where it will fluoresce upon decaying to a lower energy state. To resolve the target specie's VDF, a narrow, with respect to the width of the expected distribution, band laser is scanned across the natural absorption line. The fluorescence intensity versus laser frequency measurement is a direct measure of the absorption line shape.

When scanning across the natural absorption line, the target species sees Doppler shifted light with respect to the particle's rest frame. Assuming no bulk flow, particles moving with small velocities will be excited near the natural absorption line and particles with greater velocities will be excited further away from the absorption line. For a Maxwellian distribution of particles, the Doppler broadening of the absorption line takes the form of

$$
I(\nu)=I\left(\nu_{0}\right) \exp -\left[m c^{2}\left(\nu-\nu_{0}\right)^{2} / 2 k_{B} T \nu_{\alpha}^{2}\right]
$$

where $I(\nu)$ is the absorbed photon flux as a function of frequency $\nu, I_{0}$ is the maximum photon flux absorption, $\nu_{0}$ is the average frequency of the transition when viewed from the laboratory frame and $\nu_{\alpha}$ is the frequency of the natural absorption line.

The overall width of an absorption line is affected by several mechanisms including, but not limited to, natural line width, Stark broadening, power broadening, Doppler broaden-

ing and Zeeman broadening. ${ }^{27}$ For the measurements presented in this work, only Doppler broadening plays a significant role.

Different absorption lines have varying excitations schemes. If an atom, ion or molecule is 
excited from a ground or metastable state and then decays back to its original state, then it is called a two state, or resonant, LIF scheme. Non resonant LIF occurs when the decay from the excited state is to a state different than the original ground or metastable state. The non resonant LIF scheme utilized in this work for Ar II LIF is shown in Figure 3.1(a). For this scheme, the Ar II $3 d^{4} F_{7 / 2}$ metastable state is pumped to the $4 p^{4} D_{5 / 2}$ state using $668.6138 \mathrm{~nm}$ photons. That state decays to the $4 s^{4} P_{5 / 2}$ state by emitting $442.72 \mathrm{~nm}$ photons. The scheme chosen for Ar I is shown in Figure 3.1(b). This LIF scheme is somewhat unconventional in that the $4 s\left({ }^{2} P_{3 / 2}\right)_{1}$ state, the target state, is not metastable. The target state is populated directly by excitations from the ground and through electron impact transfers from nearby metastable states, $4 s\left({ }^{2} P_{3 / 2}\right)_{2}$ and $4 s^{\prime}\left({ }^{2} P_{1 / 2}\right)_{1}$. Work by Keesee et al. ${ }^{3}$ showed that because of its dependence on collisional mechanisms to populate the initial state, this Ar I scheme is not effective at low, $<10 \mathrm{~m}$ Torr, operating pressures.



(a) Ar II scheme

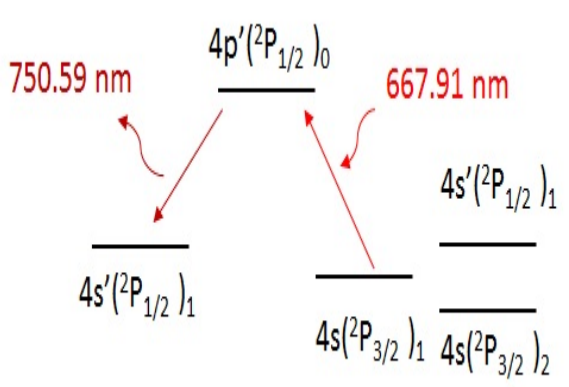

(b) Ar I scheme

Figure 3.1: Argon LIF excitation schemes for (a) Ar II and (b) Ar I. 


\subsection{Experimental Apparatus}

A sketch of the LIF diagnostic apparatus used for this work is shown in Figure 3.2. The light source chosen for this system was a TOPTICA TA 100 diode laser. The TOPTICA diode laser consists of an external cavity diode laser seeding a tapered amplifier. The system produces up to $500 \mathrm{~mW}$ of power and has a mode hop free tuning range of $50 \mathrm{Ghz}$. The diode laser system was chosen because it provides access to Ar I, Ar II and He I absorption lines. Due to its compact nature, the diode laser system allows the LIF diagnostic to be highly portable. After the diode, a $60 \mathrm{~dB}$ optical isolator protects the diode laser system by eliminating back reflections from downstream optics.

The wavelength of the laser light is continuously monitored with a High Finesse WS7 UVII wavemeter. Before being steered into the plasma chamber with mirrors, the laser beam is modulated with an acousto-optic modulator $(\mathrm{AOM})$ at $10 \mathrm{kHz}$. The light injected into the plasma expansion chamber is linearly polarized and oriented parallel to the magnetic field, mitigating the effects of Zeeman broadening. ${ }^{27}$ The fluorescent emission from the pumped excited state was collected and transported by a multimode optical fiber, passed through a $1 \mathrm{~nm}$ bandwidth filter centered around fluorescence wavelength and measured by a narrow band, high-gain, Hamamatsu photomultiplier tube (PMT). To further get rid of background light and mitigate electronic noise, a Stanford Research SR830 lock-in amplifier is used to eliminate signals uncorrelated with the laser modulation. 


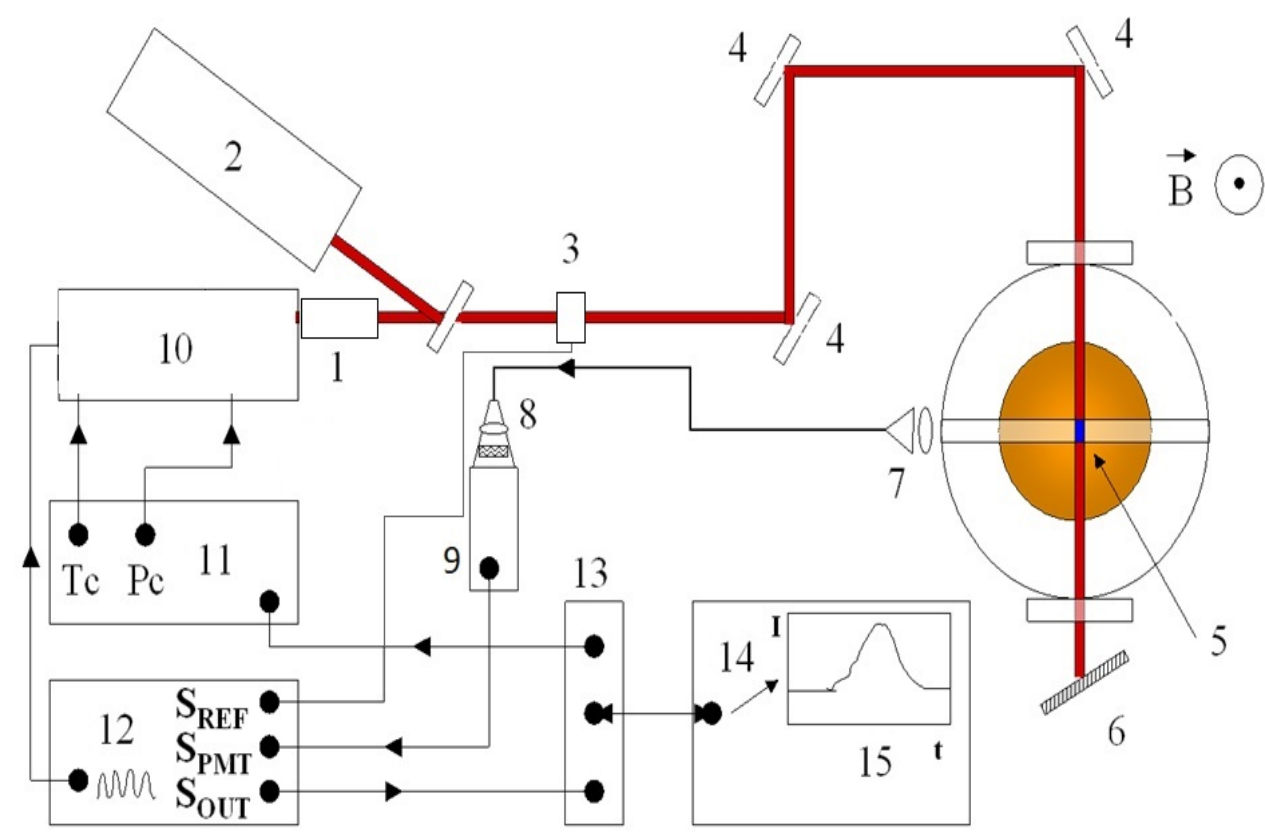

Figure 3.2: A sketch of the LIF diagnostic setup

1) Optical isolator 2)Wavemeter 3)Acusto-optic modulator 4)Steering mirrors 5)LIF volume 6)Beam dump 7)Collection Optics 8)Narrowband filter 9)PMT 10)Diode Laser 11)Laser controller 12)Lock-in amplifier 13)DAQ card 14)Laptop PC 15)Signal

\subsection{Data Analysis}

An example of an Ar II LIF VDF measurement is shown in Figure 3.3. The $x$ axis is in terms of $\Delta \nu$, where $\Delta \nu=\nu-\nu_{\alpha}$, and $\nu_{\alpha}$ is the natural absorption frequency. The distribution is fit to a Gaussian expression

$$
I(\nu)=I\left(\nu_{0}\right) \exp \left[-\left(\nu-\nu_{0}\right)^{2} / \alpha_{s} T_{i}\right]
$$

where $\alpha_{s}=2 e \nu_{\alpha} / m c^{2}$. For argon ions and neutrals respectively,

$$
\alpha_{A r I I}^{-1}=.092495 \mathrm{eV}(\mathrm{GHz})^{-2}
$$




$$
\alpha_{A r I}^{-1}=.092384 e V(\mathrm{GHz})^{-2} .
$$

The amplitude of the distribution is proportional to the excited state's density and is proportional to the relative densities from measurement to measurement. LIF can be calibrated to measure absolute densities, but the process is extremely complicated.



Figure 3.3: A typical LIF measurement of Ar II at 700 Watts rf power and a magnetic field strength of 300 Gauss. The temperature of the above distribution is $0.20 \mathrm{eV}$, calculated from the fit of the ion velocity distribution.

\subsection{Measurements}

For these experiments, we were interested in exploring plasma parameters where conducting LIF measurements was challenging. Based on previous work by Keesee, ${ }^{2,3}$ we know that the signal of the Ar I scheme becomes small at low pressures. Shown in figures 3.4(a)-3.6(b) are 
Ar II and Ar I LIF measurements at a fill pressure of 17.6 mTorr and decreasing magnetic field strengths. Varying the magnetic field strength changes the plasma's operating pressure for each measurement.

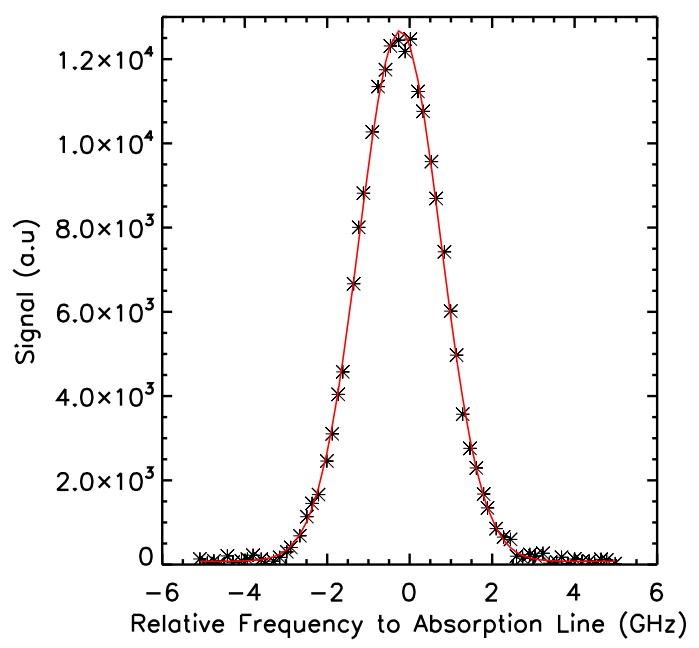

(a) $\operatorname{Ar}$ II

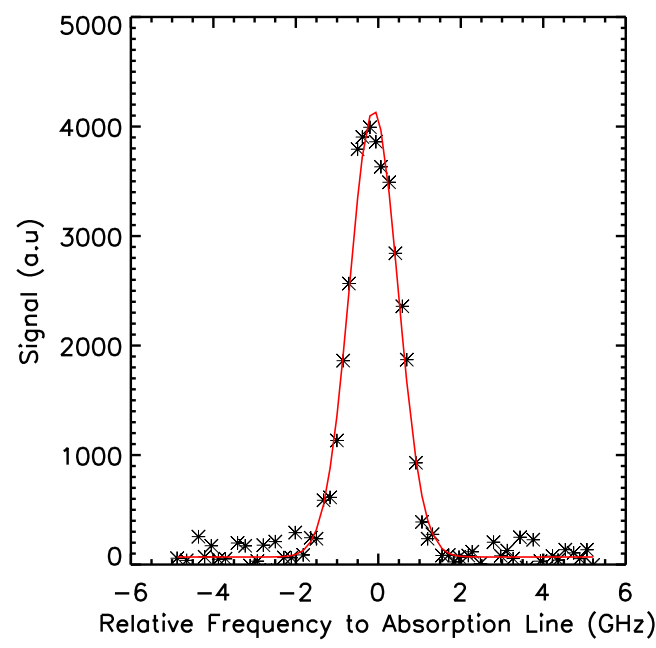

(b) Ar I

Figure 3.4: LIF measurements of (a) Ar II and (b) Ar I at $P=5.4$ mTorr, $R F=300 \mathrm{~W}, B=860 \mathrm{G}$ and Mass flow $=5$ SCCM.

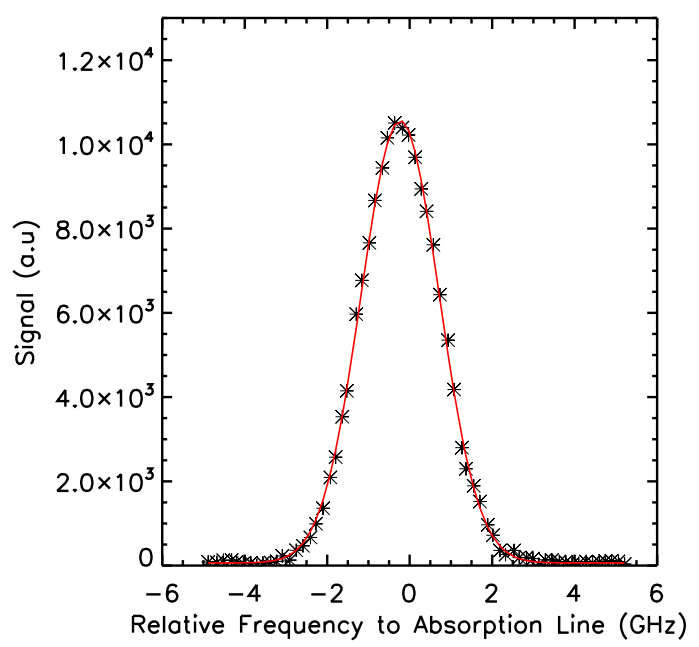

(a) Ar II

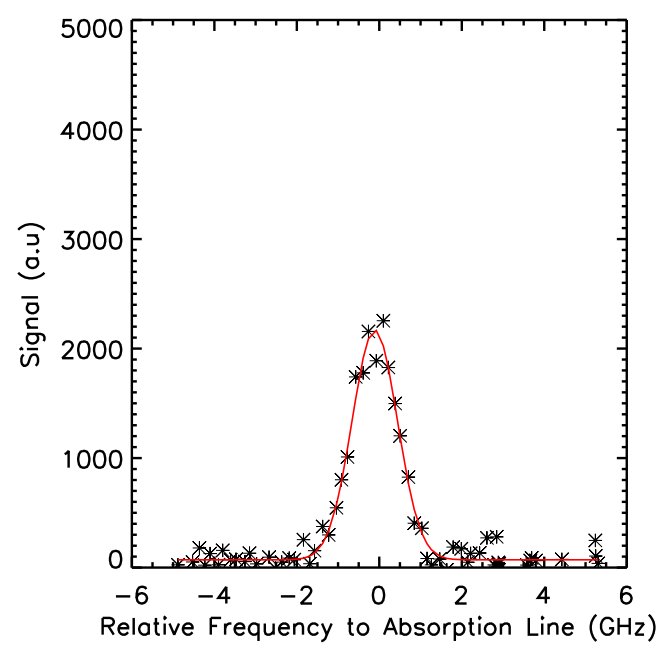

(b) Ar I

Figure 3.5: LIF measurements of (a) Ar II and (b) Ar I at $P=5.6 \mathrm{mTorr}, R F=300 \mathrm{~W}, B=800 \mathrm{G}$ and Mass flow $=5$ SCCM. 


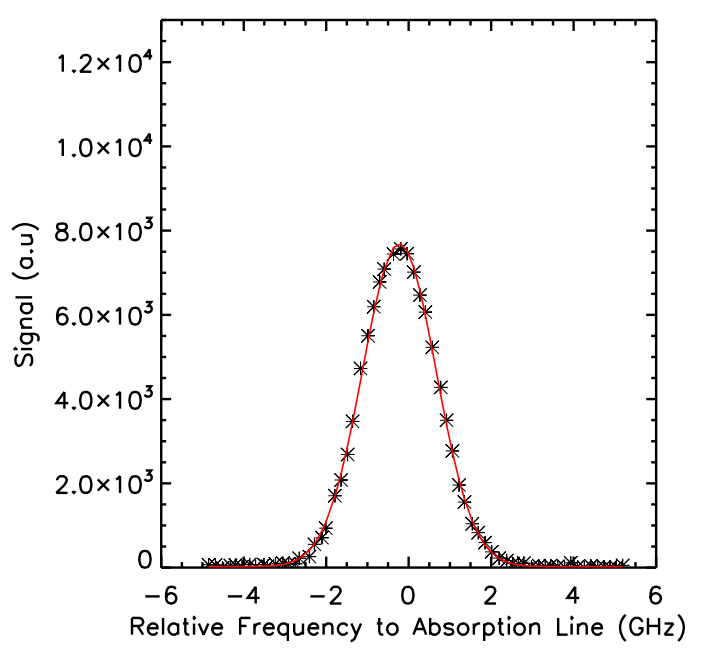

(a) Ar II

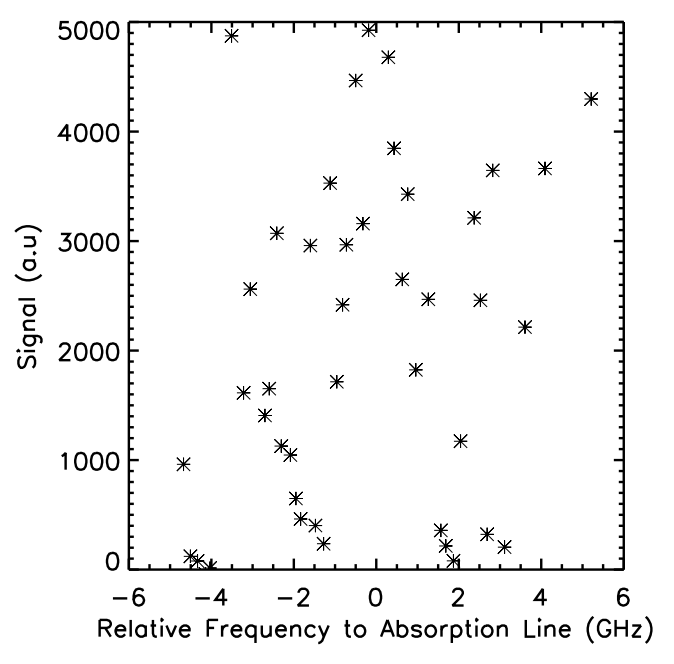

(b) Ar I

Figure 3.6: LIF measurements of (a) Ar II and (b) Ar I at $P=7.3$ mTorr, $R F=300 \mathrm{~W}, B=750 \mathrm{G}$ and Mass flow $=5$ SCCM.

With decreasing magnetic field, we observe a decrease in the perpendicular argon ion temperature, in agreement with previous experimental findings in helicon plasmas. ${ }^{28}$ For argon ions, signal was clearly resolvable from background noise with a one second integration time on the lock-in amplifier. At such high signal to noise levels, measurements required two to three minutes per distribution. For the argon neutral LIF measurements presented in this work, a ten second integration was necessary to distinguish signal from noise. Each measurement of the neutral velocity distributions required approximately forty-five minutes. When the magnetic field was decreased to 750 Gauss, the argon neutral absorption line was no longer distinguishable from the background. 


\section{Chapter 4}

\section{CW-CRDS}

Measurements of velocity distribution functions can provide valuable insights into a number of physical processes. While an assortment of probe diagnostics are commonly employed to measure VDFs, any material inserted into a plasma will interact with and alter the region of interest. Optical diagnostics can be used to measure VDFs without perturbing the plasma. In the previous chapter we discussed LIF and showed an example of plasma parameters for which LIF is ineffective for measurements of Ar I VDFs. Here we discuss a diagnostic technique that could be used to measure VDFs where traditional optical diagnostics, like LIF, falter: continuous wave cavity ring-down spectroscopy (CW-CRDS). 


\subsection{Absorption spectroscopy}



Figure 4.1: A sketch of a typical absorption spectroscopy experiment. Figure adapted from Berden and Engeln. ${ }^{29}$

One possible alternative to LIF is absorption spectroscopy. A sketch of a typical single pass absorption spectroscopy experiment is shown in Figure 4.1. The apparatus consists of a laser, an absorption cell of length $L$, and a detector to measure the intensity of the laser light. The absorption cell normally consists of a pressurized chamber capped with two windows. Laser light, tuned to a natural absorption line, passes through the filled absorption cell and is measured by the detector. The resultant intensity measured after the absorption cell follows the Beer-Lambert law

$$
I_{t}=I_{0} \exp [-\alpha L]
$$

where $I_{0}$ is the intensity measured with an empty cavity and $\alpha$ is the absorption coefficient across the absorption cell of length $L$. The absorption coefficient is defined as

$$
\alpha(\nu)=N \sigma(\nu)
$$


assuming all absorbers are from a single species. Here, $N$ is the number density of absorbers, $\sigma$ is the absorption cross-section and $\nu$ is the frequency of the laser light.

If a narrow band laser is swept across the natural absorption line of a species, the Doppler broadened absorption line corresponds to the line-integrated velocity distribution. The absolute, line integrated density of the absorbing species is obtained from the measurement by the relations of Equations 4.1 and 4.2.

The line-integrated nature of absorption spectroscopy is in contrast to the spatially resolved nature of LIF. Any information, temperature or density, is integrated over the entire path-length of the laser through the absorption cell. Single pass absorption spectroscopy also suffers from a lack of sensitivity. In the absence of a large number of absorbers the transmitted intensity may be indistinguishable from the intensity measured from an empty absorption cell. Laser drifts and fluctuations in laser intensity also limit the sensitivity obtainable by single pass absorption spectroscopy.

\subsection{Pulsed Cavity Ring-Down Spectroscopy}

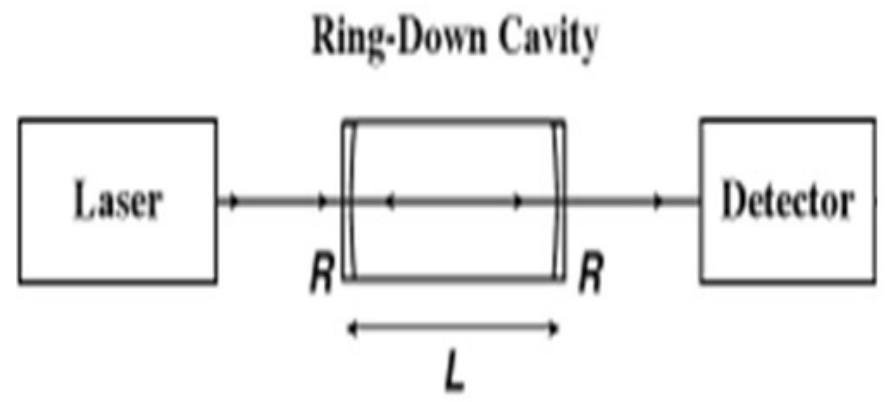

Figure 4.2: A sketch of a typical pulsed cavity ring-down spectroscopy diagnostic. Figure adapted from Berden and Engeln. ${ }^{29}$ 
As discussed in the previous section, single pass absorption spectroscopy has poor signal to noise if there are laser intensity fluctuations and a limited number of absorbers. Pulsed cavity ring-down spectroscopy (P-CRDS) is able to address both of these limiting factors. A sketch of a typical P-CRDS diagnostic is given in Figure 4.2. In a P-CRDS experiment, the windows of the absorption cell are replaced by highly reflective (99.99\% reflectivity), concave mirrors of radius of curvature $R$. A pulsed laser is fired through the "ring-down cavity" and the intensity transmitted through the cavity is measured with a photo detector.

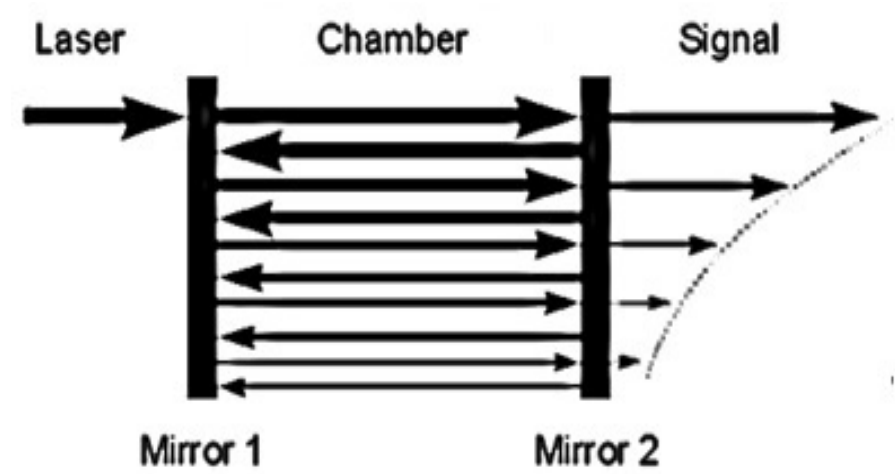

Figure 4.3: Pulsed laser beam "bouncing" in ring-down cavity.

To conceptualize how the laser pulse interacts with the ring-down cavity it is useful to think of a pulse of light "bouncing" back and forth between the two mirrors (Figure 4.3). The transmitted intensity of the beam still obeys the Beer-Lambert law so the initial trip through the ring-down cavity results in an intensity given by

$$
I_{0}=I_{l a s e r} T^{2} \exp [-\alpha d]
$$

where $T$ is the transmission coefficient of the highly reflective mirrors and $d$ is the path length of the laser through the absorbing medium. The next emission detected will be after 
the beam makes its first round trip in the cavity and is given by

$$
I_{1}=I_{0} R^{2} \exp [-2 \alpha d]
$$

where $R$ is the reflectivity of the mirrors. Each successive bounce results in another round trip through the chamber and the intensity emitted after $n$ trips is

$$
I_{n}=I_{0} R^{2 n} \exp [-2 n \alpha d]
$$

The intensity as a function of time is obtained by replacing the number of round trips $n$ with the time that it takes for the beam of light to complete a circuit through the ring-down cavity, $t=n 2 L / c$. With this substitution and writing $R^{2 n}$ as $\exp [2 n \ln (R)]$, Equation 4.5 becomes

$$
I_{t}=I_{0} \exp \left[\frac{t c}{L}(\ln (R)-\alpha d)\right]
$$

For values of $R$ close to $1, \ln (R) \approx-(1-R)$ we can rewrite Eqn. 4.6 as

$$
I_{t}=I_{0} \exp \left[-\frac{t c}{L}(1-R+\alpha d)\right]
$$

The time it takes for the intensity to exponentially decay to $1 / e$ of its initial value is called the ring-down time $\tau$ and is defined as

$$
\tau=\frac{L}{c(1-R+\alpha d)}
$$

It is important to note that in the $\tau$ defined here, it is assumed that only a single species of absorbers are present and small changes in reflectivity due to laser frequency changes are 
ignorable.

To determine the line integrated absorption coefficient $\alpha$, on and off resonance ringdown time measurements are required. The resonant ring-down times obey Eqn.4.8, and off resonance cases simplify to

$$
\tau_{0}=\frac{L}{c(1-R)}
$$

$\alpha$ is then calculated from the difference in the cavity losses $(k=1 / c \tau)$ between the on and off resonance measurements.

$$
\alpha \frac{d}{L}=k-k_{0}=\frac{1}{c \tau}-\frac{1}{c \tau_{0}}
$$

Once the absorption coefficient is known, the line-integrated density of the target species is calculated from Eqn.4.2.

We can infer the sensitivity of the P-CRDS technique by writing Eqn.4.10 in the form of the minimal detectable absorption

$$
\left[\alpha \frac{d}{L}\right]_{\min }=\Delta k_{\min }=\frac{1-R}{L}\left[\frac{\Delta \tau}{\tau_{0}}\right]_{\min } .
$$

It is important to take note of a few things when discussing the sensitivity of the P-CRDS technique. First, the effective path-length of the laser through the absorbing medium is orders of magnitude larger than the length of the ring-down cavity. By making thousands of round trips through the sample, even trace amounts of absorbers are detectable. It is also important to point out that the absorption coefficient is calculated from the cavity losses and is independent of the initial laser intensity! Despite the increased sensitivity, P-CRDS cannot be used to measure the Doppler broadened absorption line like the single pass absorption technique described earlier. Pulsed laser bandwidths are rarely narrow enough to resolve 
Doppler broadened absorption lines (Figure 4.4), especially for the low temperature plasmas of interest in this work.

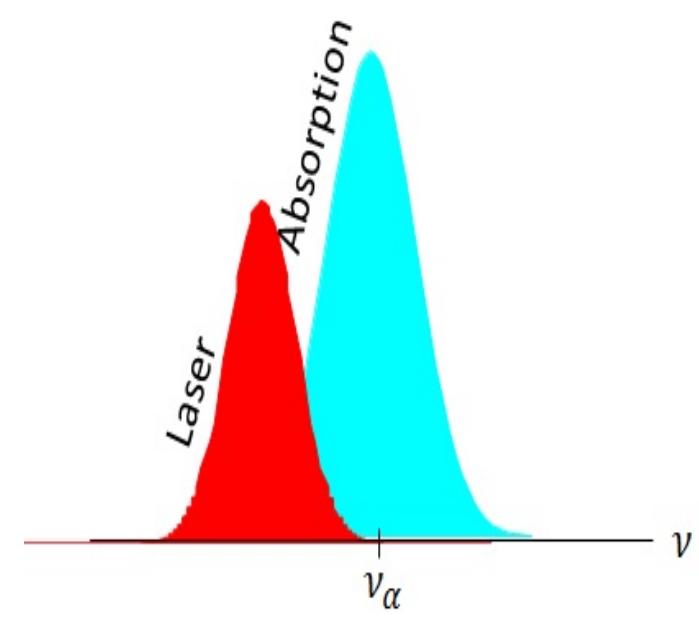

Figure 4.4: Cartoon of pulsed laser bandwidth compared to a Doppler broadened distribution function. Most pulsed lasers systems have bandwidths too broad to resolve the Doppler broadened distributions measured in this work, which are on the order of a few GHz. Here $\nu_{\alpha}$ is the natural absorption frequency.

\subsection{Continuous Wave Cavity Ring-down Spectroscopy}

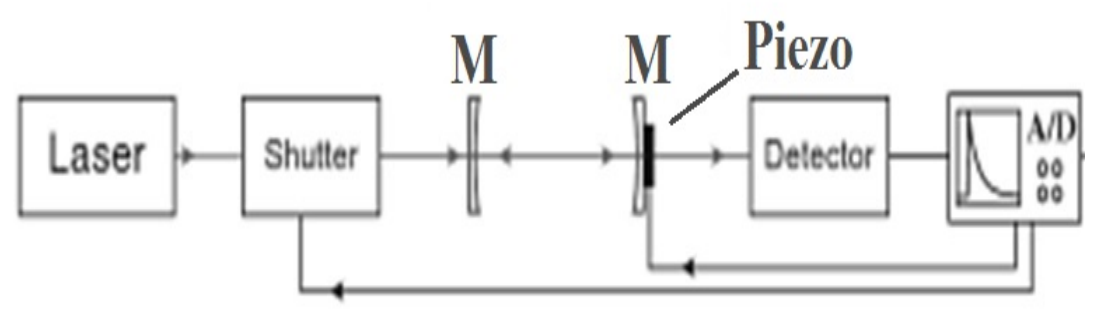

Figure 4.5: A sketch of a typical CW-CRDS diagnostic setup.

Figure adapted from Berden and Engeln. ${ }^{29}$ 
To resolve a Doppler broadened absorption line and obtain an accurate measurement of the absorption line width a narrow band width laser is required. Narrow band lasers are available for cavity ring-down measurements, but the experimental setup is more challenging because the lasers are typically continuous wave. The typical setup for continuous wave cavity ringdown spectroscopy (CW-CRDS) is shown in Figure 4.5. As in the P-CRDS apparatus, the ring-down cavity is formed by two highly reflective mirrors. In a typical CW-CRDS experiment one of the mirrors is placed on a piezo-electric transducer (PZT) that allows the cavity length to be modulated. Laser light is shined into the cavity and when the resonance condition is met, evident by a dramatic increase in transmitted light out of the ring-down cavity, the incident light is blocked by the shutter. After the light is extinguished, the ring-down time in the cavity is measured as described in the previous section.

Achieving the resonance condition with a narrow line width laser is experimentally challenging. Resonance is achieved when a standing wave is formed in the cavity so that constructive interference results in a significant gain in transmitted intensity. As noted earlier, for a pulsed laser this condition is met with very little effort, but for a narrow band cw laser detailed understanding of optical cavities is necessary to achieve the resonant condition and optimize the CW-CRDS system.

\subsubsection{Free Spectral Range}

To create a standing wave the cavity has to accommodate an integer number of half wavelengths, such that $L=n \lambda / 2$. When this condition is met and constructive interference occurs in the cavity, it is referred to as a longitudinal mode. When discussing optics it is often beneficial to define key parameters in terms of frequency. The distance between two 
longitudinal modes, $\Delta n=1$, is

$$
\delta \nu_{F S R}=c / 2 L
$$

where $L$ is the length of a non-confocal cavity and $\delta \nu_{F S R}$ is the free spectral range.

\subsubsection{The Cavity Finesse}

The finesse of an optical cavity is a measure of the cavity's ability to maintain a resonance condition. The higher the finesse, the harder it is to maintain a resonance condition. It is directly related to the effective path length of the optical cavity $L /(1-R)$, where $R$ is the reflectivity of the mirrors. The finesse of a ring-down cavity plays a key role in determining the sensitivity (Eqn. 4.11) of a CW-CRDS diagnostic. The finesse is usually written

$$
F=\frac{\pi \sqrt{R}}{(1-R)}
$$

\subsubsection{Cavity Stability}

The stability of an optical cavity determines whether the injected light will eventually "bounce" out the cavity during successive round trips. If the light is effectively "trapped" in the ring-down cavity, the cavity is stable. The stability of a cavity is defined by its length $L$ and the radius of curvature of the mirrors $r_{n}$. By using ray transfer matrices ${ }^{30}$ it can be shown that a cavity must satisfy the condition

$$
0<\left[1-\frac{L}{r_{1}}\right]\left[1-\frac{L}{r_{2}}\right]<1
$$


It is often convenient to introduce stability parameters, $g_{n}=1-L / r_{n}$, that define both the curvature and length of a cavity. Rewriting Eqn. 4.14 in terms of g-parameters gives

$$
0<g_{1} g_{2}<1
$$

For the non confocal cavity used in this work, both mirrors have the same radius of curvature so the stability conditions become $0<L<r$ and $r<L<2 r$.

\subsubsection{Cavity Mode Structure}

In a cavity of length $L$, the frequency of resonant modes in terms of the g-parameters is

$$
\nu_{q m n}=\frac{c}{2 L}\left[q+\frac{n+m+1}{\pi} \arccos \left(\sqrt{g_{1} g_{2}}\right)\right]
$$

where $q, m$ and $n$ are integers. ${ }^{29}$ The longitudinal $(q)$ and transverse $(m$ and $n)$ mode indices are used to distinguish characteristics of the electromagnetic field of each mode. The separation of longitudinal modes with the same $m$ and $n$ is given by $\delta \nu_{F S R}$ (Figure 4.6).

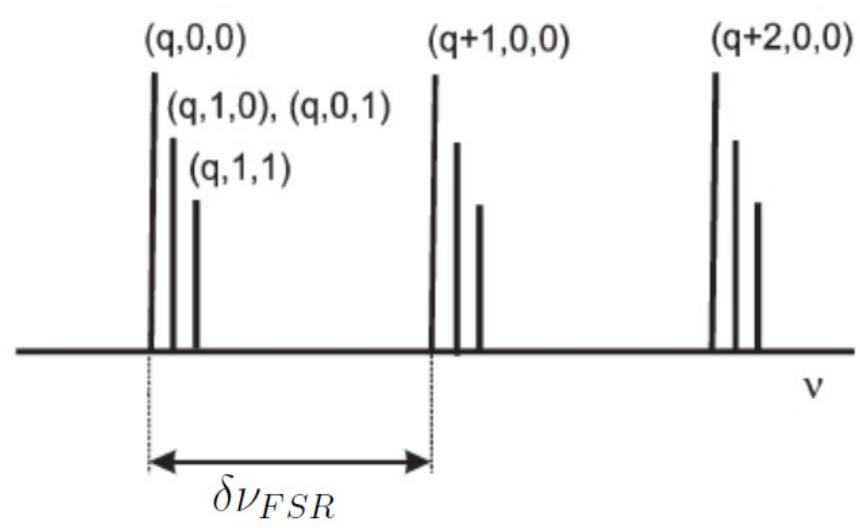

Figure 4.6: Spacing of longitudinal modes. Figure adapted from Mazurenka. ${ }^{31}$ 
Modes with different $m, n$, but the same $q$ are called transverse modes and are usually conventionally labeled as $T E M_{m n}$ modes. Each of these modes have a distinct cross sectional intensity profile and travel unique paths through the optical cavity (Figure 4.8).
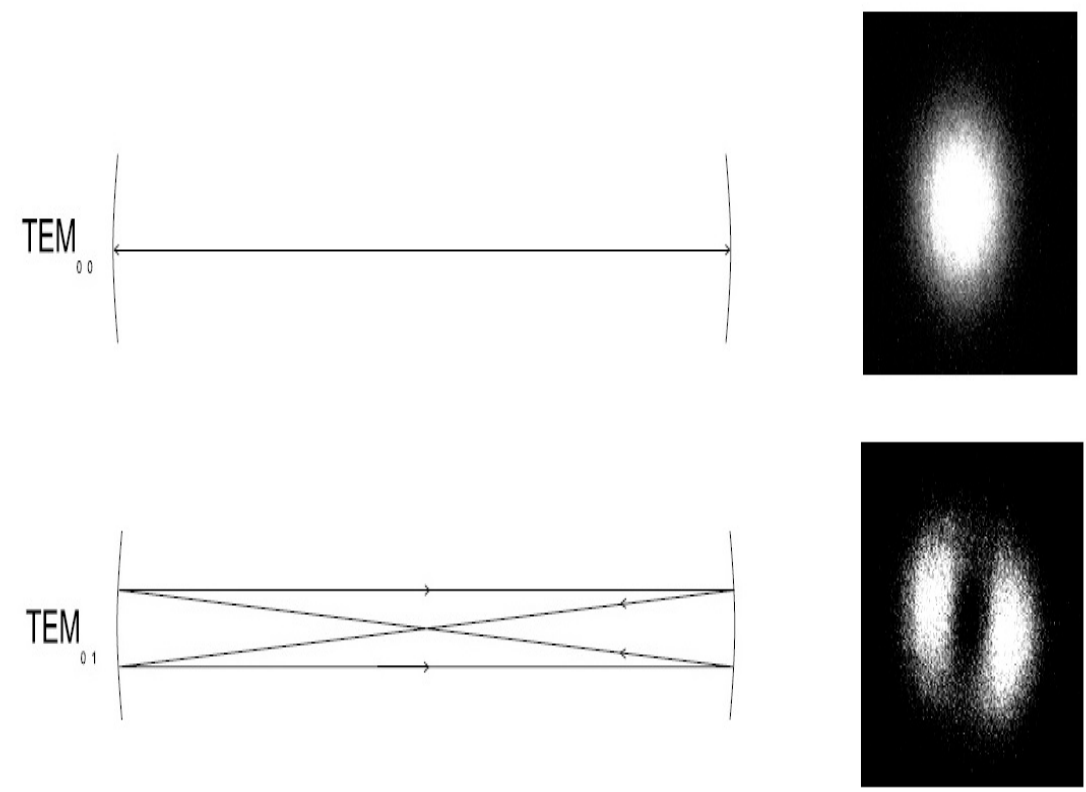

Figure 4.7: Schematics of the $T E M_{00}$ and $T E M_{01}$ path through the optical cavity (left) and their respective intensity profiles (right). Figure adapted from Hemerik. ${ }^{32}$

\subsubsection{The Cavity Spot Size}

Optical cavities are defined by the spot size of the lowest order, $T E M_{00}$, Gaussian modes.

The spot size on the mirrors is

$$
\omega_{1,2}=\sqrt{\frac{L \lambda}{\pi}}\left[\frac{g_{2,1}}{g_{1,2}\left(1-g_{1} g_{2}\right)}\right]^{.25}
$$


and the spot size at the focal point of the cavity is

$$
\omega_{0}=\sqrt{\frac{L \lambda}{\pi}}\left[\frac{g_{1} g_{2}\left(1-g_{1} g_{2}\right)}{\left(g_{1}+g_{2}-2 g_{1} g_{2}\right)^{2}}\right]^{.25}
$$

Here it is important to note that the spot size $\omega$ corresponds to a radial distance from the beam center such that $I(\omega)$ is equal to $I(0) / e^{2} \cdot{ }^{29}$

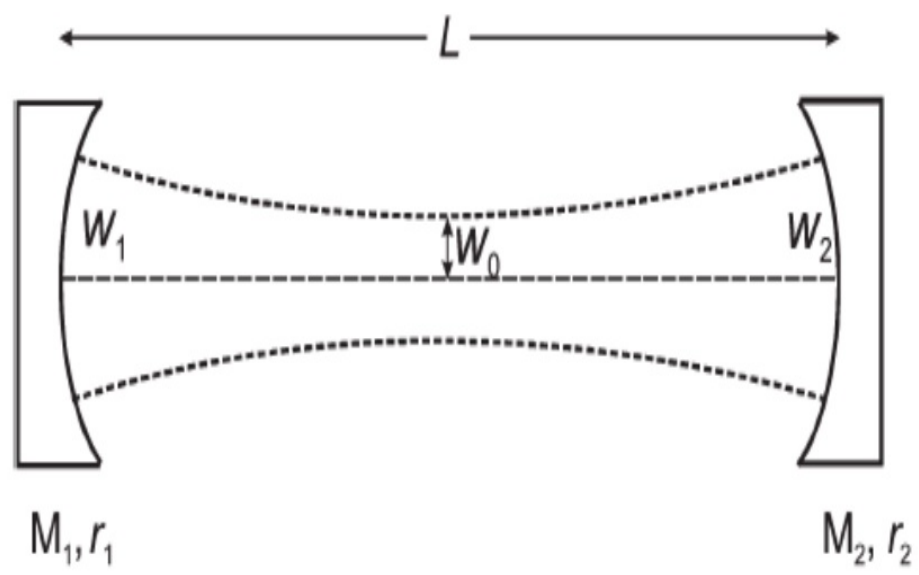

Figure 4.8: The spot sizes in an optical cavity. $W_{1}$ and $W_{2}$ are the spot sizes on the surface of the mirrors $M_{1}$ and $M_{2}$ respectively. $W_{0}$ is the spot size of the beam at the cavities' focal point. ${ }^{31}$

\subsubsection{Mode-Matching}

The challenge in CW-CRDS is to preferentially couple to a specific mode. Typically the Gaussian $T E M_{00}$ mode is chosen in CRDS experiments. A single mode is critical because each TEM mode has a distinct path through the cavity, meaning each mode will have a different characteristic $\tau$. To precisely calculate the absorption coefficient $\alpha$ we need $\tau_{0}$ and $\tau(\nu)$ measurements for the same specific mode.

Mode coupling requires matching the location of the waist of the injected laser beam to 
the focal point of the cavity. The injected beam spot size must also match the beam spot size as defined by the parameters of the optical cavity. It is optimal for the injection beam to have a Gaussian profile to preferentially excite the $T E M_{00}$ mode. Shaping the injection beam is accomplished with a spatial filter comprised of two focusing lenses and a pinhole or by coupling the light into a single mode fiber. The resulting Gaussian beam is then focused down to the correct beam waist at the optical cavity's focal spot.

Transforming the injection beam to the correct spot size at a precise location is nontrivial, but the necessary calculations for positioning the lens are easily performed using the properties of Gaussian beams and ray transfer matrices (Figure 4.9). ${ }^{30}$

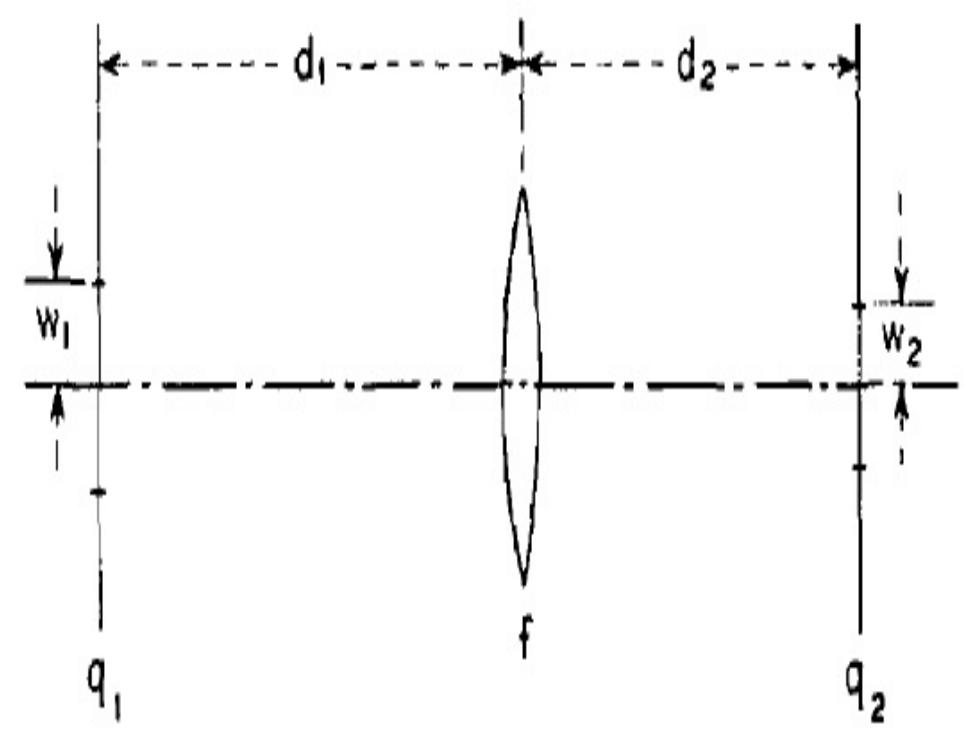

Figure 4.9: Diagram for distances and parameters for a beam transformation by a thin lens. ${ }^{30}$

Here $d$ is the distance from the lens to the initial and transformed beam's waist, $W$ is the spot size of the beam, $f$ is the focal length of the lens and $q$ is a complex beam parameter defined by the spot size and radius of curvature of the beam at the given location.

The distances $d_{1}$ and $d_{2}$ have unique solutions for a given lens with focal length $f$ given 
by

$$
\begin{aligned}
& d_{1}=f \pm \frac{\omega_{1}}{\omega_{2}} \sqrt{f^{2}-f_{0}^{2}} \\
& d_{2}=f \pm \frac{\omega_{2}}{\omega_{1}} \sqrt{f^{2}-f_{0}^{2}}
\end{aligned}
$$

where $f_{0}=\pi \omega_{1} \omega_{2} / \lambda$. Here $f_{0}$ is a characteristic length defined by the beam waist to be matched. A solution exists only for lenses where $f>f_{0}$. More intricate calculations can be performed that take into account lens thickness and transmission through the CRDS mirrors, but it is often a case of diminishing returns. Due to the frustrating lack of perfection in real physical systems, the calculated distances need to be adjusted by up to $10 \%$ to experimentally optimize the mode matching. ${ }^{33}$

\subsection{CW-CRDS Experimental Apparatus, MARK-I}

In this section, we briefly discuss the MARK-I CW-CRDS setup and its key components. An extensive review of the MARK-I CW-CRDS setup can be found in Thakur. ${ }^{19}$ A diagram of the experimental apparatus is given in Figure 4.10. The same Toptica TA 100 diode laser described in Section 3.2 was used for the MARK-I CW-CRDS experiments. A $60 \mathrm{~dB}$ optical isolator was placed after the diode laser to prevent back reflections from damaging the diode or amplifier system. After passing through the optical isolator the light is diffracted and modulated with an acousto-optic modulator (AOM). All but the first order diffraction, $m=1$, light is blocked with an iris. The $m=1$ beam is then steered into a spatial filter to make the beam's intensity pattern more Gaussian. The spatial filter consisted of two 2" plano-convex lenses and a 50 micron pinhole. The light is focused down onto the pinhole and the diffracted light is then collimated by the second lens. Then the beam, which has 
a Newton's rings intensity pattern, is sent through an iris that blocks all but the central, Gaussian, beam.



Figure 4.10: Schematic of the MARK-I CW-CRDS experiment.

$I$ :Iris, $M$ :Beam steering mirror, $L:$ Spatial filter lens, $P H$ : Pinhole, $L_{M}$ : Mode matching lens, $H M$ : Highly reflective mirrors, AOM:Acousto-optic modulator, PZT:Piezo electric transducer. ${ }^{19}$

Next a $75 \mathrm{~cm}$ plano-convex "mode matching" lens is used to focus the beam to the center of the $85 \mathrm{~cm}$ optical cavity. The optical cavity is formed by two highly reflective mirrors. To perform measurements in the CHEWIE plasma source, special mounts were made to house the mirror and allow for cavity adjustments to be made while the system was under vacuum (Figure 4.11). One of the mirrors that comprised the optical cavity was mounted on a Physik Instruments (PI) piezo electric transducer (PZT) stage that was driven by a $10 \mathrm{~Hz}$ triangle wave. The PZT introduced small, periodic, modulations of the length of the optical cavity on the order of microns, facilitating resonance conditions for a range of laser frequencies. 


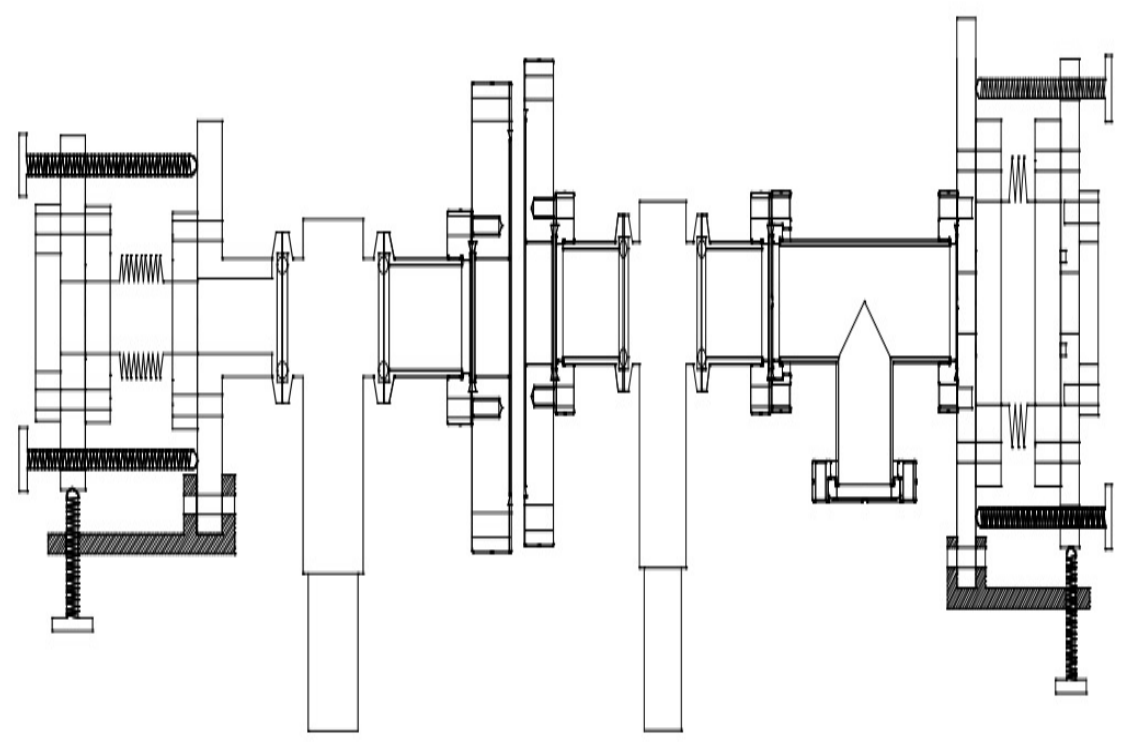

Figure 4.11: Sketches of the MARK-I vacuum mirror mounts. The MARK-I mounts were built in house and allows the mirrors to be aligned without breaking vacuum. When the CW-CRDS diagnostic is not in use the mirrors can be isolated from the expansion chambers using gate valves. ${ }^{19}$

Light transmitted through the cavity is measured by a New-Focus photo-receiver (Model2051). The photo-receiver is a silicon diode photo detector with a built in transimpedance amplifier and an adjustable bandwidth of up to $10 \mathrm{MHz}$. The signal from the photo-receiver is sent to an oscilloscope and a tracking circuit. The oscilloscope for measuring and recording ring-down times is a LeCroy WwaveRunner 604Zi. The tracking circuit (Figure 4.12) is used to extinguish the laser light once an optimal resonance condition is met. The signal from the photo-receiver is compared to an adjustable DC "threshold" voltage. If the signal voltage exceeds the threshold voltage, the TTL pulse train modulating the AOM is modified so that for the next 80 microseconds, no diffractions will be produced; effectively extinguishing the $m=1$ beam being injected into the ring-down cavity. A more detailed description and a discussion on the development of the tracking circuit is found in Thakur. ${ }^{19}$ 


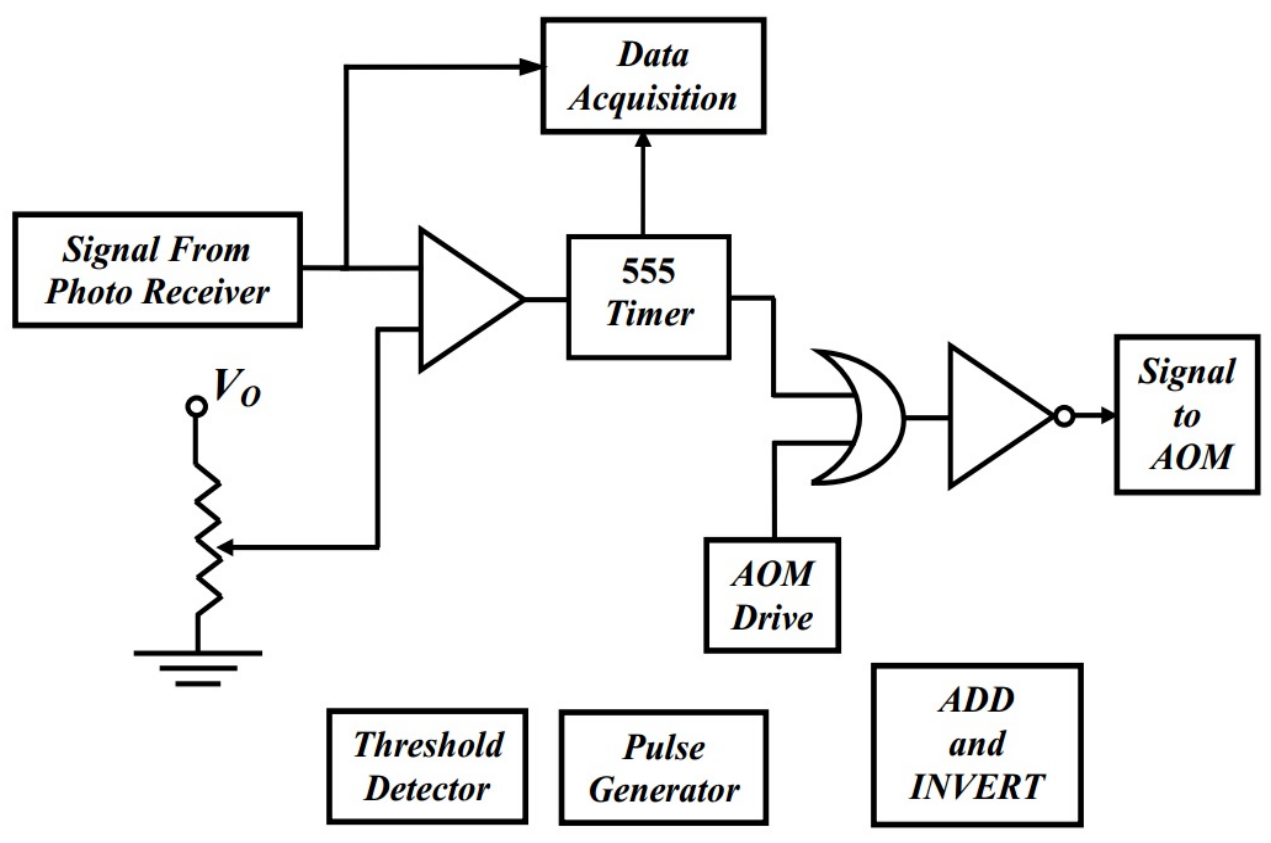

Figure 4.12: Sketch of the MARK-I tracking circuit. The tracking circuit is used to extinguish the light in the cavity once the resonance condition has been met. The circuit consists of a comparator, a 555 pulse generator, an adder and an inverter chip. ${ }^{19}$

\subsection{Initial CW-CRDS measurements}

A proof-of-principle measurement was made using the MARK-I apparatus. For this measurement the diode laser was set to a fixed frequency and the cavity length was modulated using the PZT to obtain resonance conditions. Measurements of the ring-down times $\tau$ were made with the plasma on and off to find the absorption coefficient of the plasma. To resolve the Doppler broadened absorption line, on-off plasma measurements were made at several laser frequencies near $668.6138 \mathrm{~nm}$, the same natural absorption line of Ar II used for LIF. Figure 4.13 shows the proof-of-principle measurement made with the MARK-I CW-CRDS system. The y-axis is the difference in plasma on-plasma off ring-down times in microseconds. The $\mathrm{x}$-axis is the relative frequency compared to the rest frame absorption frequency of Ar II at 
$668.6138 \mathrm{~nm}$. The distribution measurement took approximately thirty hours to complete and has a signal-to-noise of barely 2 to 1 . Only a few data points were obtained across the lineshape and the line width corresponded to uncharacteristically small perpendicular ion temperature of only $0.09 \mathrm{eV}$.

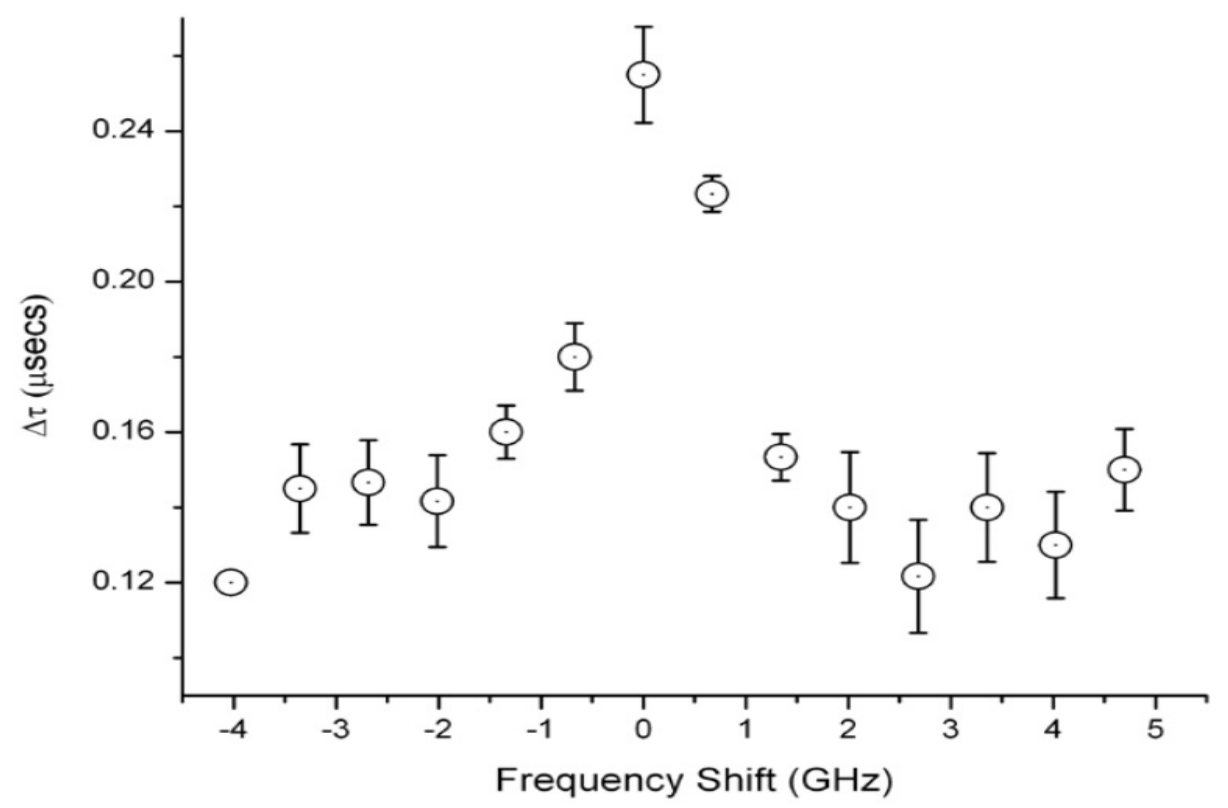

Figure 4.13: Proof of principal measurement by the MARK-I cw-crds diagnostic for Ar II. ${ }^{19}$

It is common for the quality of a CRDS measurement to be defined by

$$
\operatorname{error}=\frac{\delta \tau}{\tilde{\tau}}
$$

where $\tilde{\tau}$ is the averaged ring-down time and $\delta \tau$ is the standard deviation of the averaged ring-down times. The percent error associated with these proof-of-principle measurements was around $17 \%$. Typically, CW-CRDS diagnostics operate with an error of $<1 \%$. The MARK-I proof-of-principal measurement demonstrated wavelength dependent absorption, 
but to accurately resolve the VDF, improvements were clearly needed.

\subsection{Zeroth Order Error Propagation Analysis}

The overall errors in the MARK-I system were of course cumulative, but to be improved, each problematic element in the system was identified and upgrades implemented.

\subsubsection{Sub-Optimal Mode Matching}

As discussed in Section 4.3.6, mode matching is a rigorous process that is crucial to optimizing a CW-CRDS diagnostic. Mode matching was achieved by properly setting up the ring-down cavity and mode matching lens. While tuning the system, the highly reflective mirrors that comprise the optical cavity and the mode matching optics were aligned in such a way that the $T E M_{00}$ was the dominant mode in the cavity. If this condition was met, the emitted intensity of a $T E M_{00}$ mode resonance should exceed the intensities of higher order modes. Such optimizations not only efficiently utilize the energy present in the system, but they also allow selection of a single mode with the threshold tracking circuit. Unfortunately, small perturbations have the potential to cause a well mode matched system to drift out of alignment over time and allow higher order modes to have emission intensities comparable to the $T E M_{00}$ mode. This problem is prevalent in any CW-CRDS and affects the sensitivity of an unoptimized system.

In Figure 4.14 two distinct distributions of ring-down times are evident. This is the result of the threshold tracking circuit being unable to preferentially sample a single mode

due to insufficient mode matching. Recall Figure 4.7, it is apparent that each $T E M_{m n}$ mode has a different characteristic ring-down time $\tau$ due to its unique path through the cavity. 
Specific modes could be selectively analyzed post acquisition, but only if they were clearly distinguishable, i.e. the two distributions were separated by several standard deviations from one another.
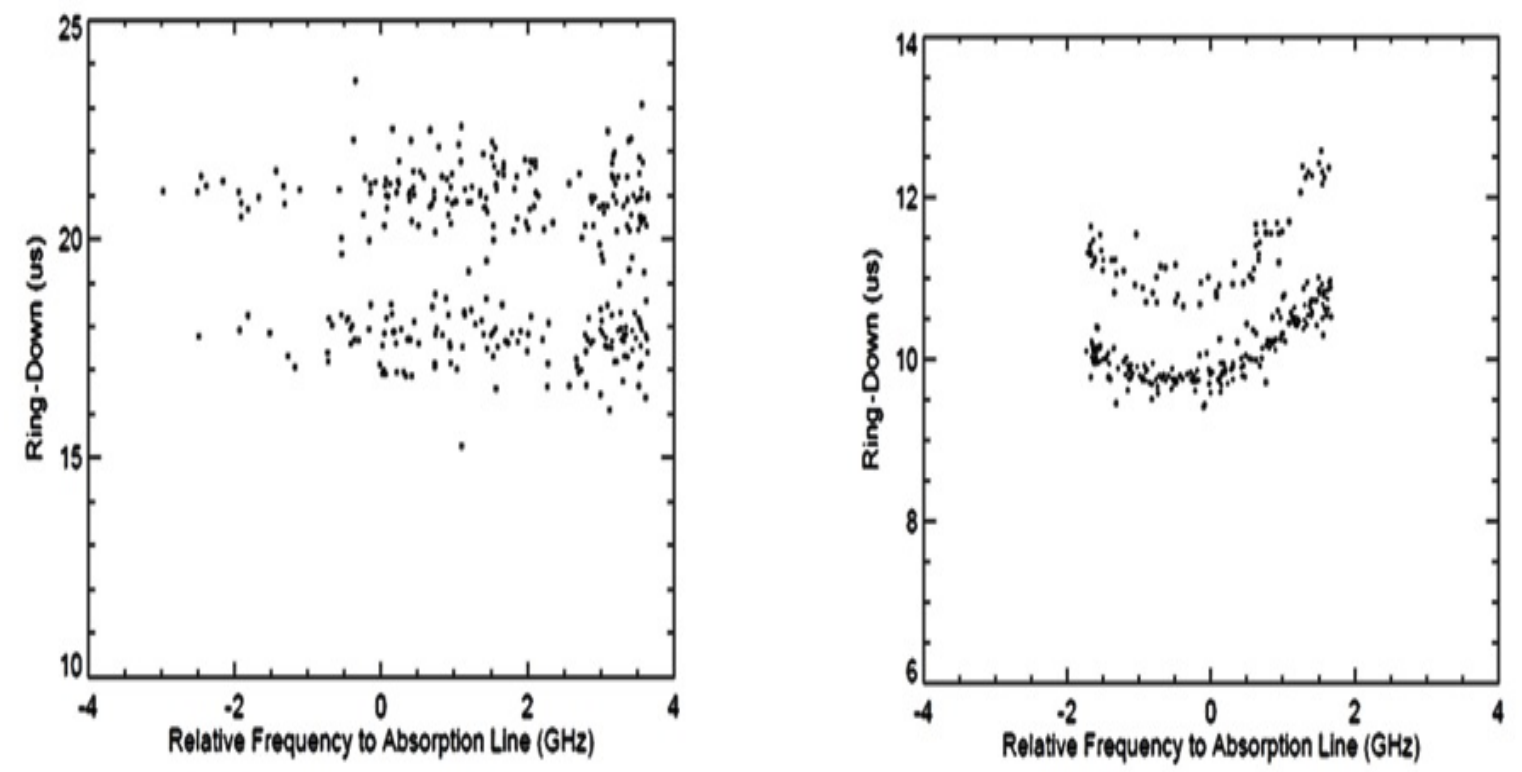

Figure 4.14: The effects of sub-optimal mode matching. The effects are present in a cavity effectively empty of absorbers (left) and in the presence of absorbers generated by the helicon plasma (right).

\subsubsection{Injection Beam Extinction}

In the MARK-I CW-CRDS system it was essential that the light from the cw laser be extinguished during the ring-down measurement. The purpose of the tracking circuit was to modify the TTL signal sent to the AOM to ensure that for the duration of a ring-down in the cavity the $m=1$ beam was not generated, effectively extinguishing the light being injected into the cavity. If the light was not completely extinguished higher order modes could be excited during a ring-down measurement or additional $T E M_{00}$ power injected (Figure 4.15). 
When this occurred, the decay curve could no longer be fit to a simple exponential curve for a unique $\tau$ value.

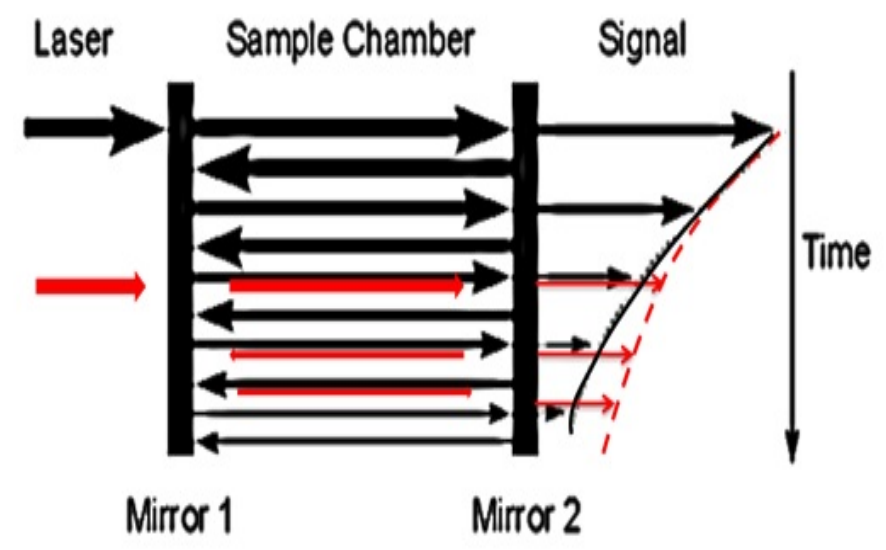

Figure 4.15: A cartoon depicting higher order mode excitation during a ring-down measurement. The dashed line indicates the sum intensity of the two resonances that can no longer be fit to a simple exponential.

The extinction ratio of the AOM plays a pivotal role in extinguishing the light injected into the cavity. It has been shown that an extinction ratio $>75 \mathrm{~dB}$ is required to mitigate the effects of higher mode excitation during a ring-down. ${ }^{34}$ Upon examining the AOM used for the MARK-I proof-of-principal measurement, it was discovered that it had been damaged and at the time of the measurement had an extinction ratio of only $15 \mathrm{~dB}$. With practice, the extinction ratio could be increased to a maximum extinction ratio of $27 \mathrm{~dB}$. By examining a histogram of ring-down times in an empty cavity with an extinction ratio of $15 \mathrm{~dB}$, two distinct distributions were observed (Figure 4.16:left). By optimizing the extinction ratio to $27 \mathrm{~dB}$ and repeating the measurement, only a single, albeit asymmetric, distribution of ringdown time was observed (Figure 4.16:right). Previously, the presence of what had appeared to be two distributions had been diagnosed as a mode matching problem. By properly diagnosing the mechanism as insufficient extinction of the injection beam, modifications 
were made that greatly increased the sensitivity of the CW-CRDS diagnostic.
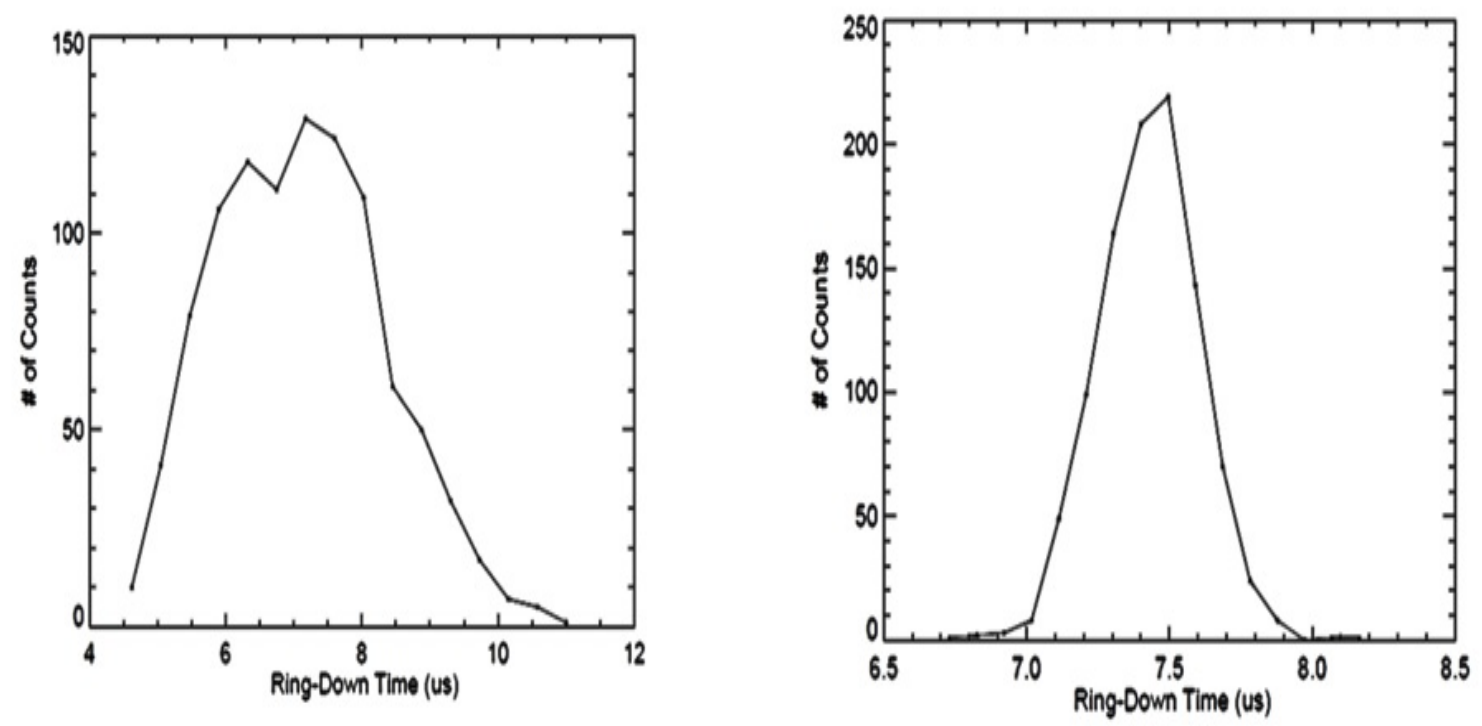

Figure 4.16: Histograms of ring-down times measured using an $\mathrm{AOM}$ with an extinction ratio of $15 \mathrm{Db}$ (left) and $27 \mathrm{~dB}$ (right).

\subsubsection{Particle Depositions on Mirrors}

It is not possible to obtain the resonance condition in the ring-down cavity if a glass window is placed between the two highly reflective mirrors. Because of this fundamental constraint, it was necessary to place the highly reflective mirrors inside the CHEWIE vacuum chamber to form the optical cavity. In helicon plasma sources, a certain amount of sputtering occurs and accumulates on optical view ports over time. The highly reflective mirrors used for the MARK-I CW-CRDS system experienced such deposition, ultimately causing irreversible losses in reflectivity. These losses resulted in shot-to-shot variations in measured ring-down times and a continually decreasing sensitivity. Figure 4.17 shows the permanent decrease in ring-down times with subsequent plasma measurements using the MARK-I CW-CRDS 
experiment. Despite the use of narrow bellows in the MARK-I vacuum mirror mounts, mirror degradation continued to occur.

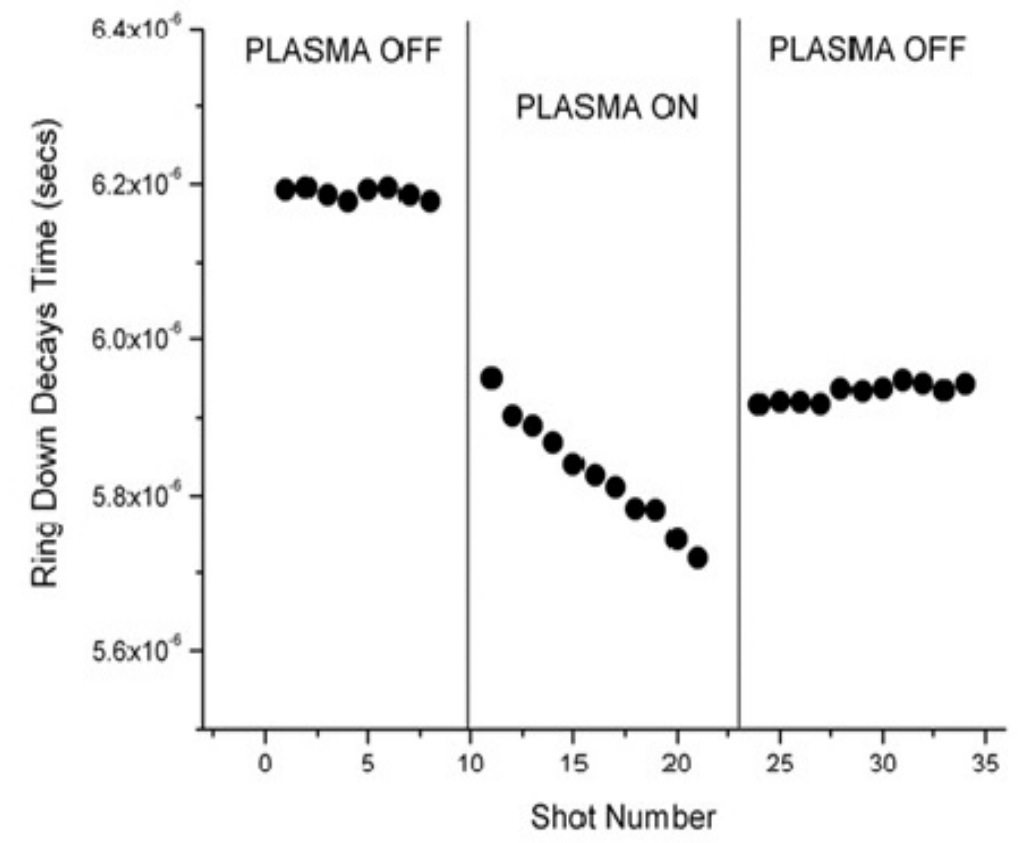

Figure 4.17: Shot-to-shot variations in ring-down measurements due to particle deposition on the highly reflective mirrors. The time between each shot was approximately five minutes. ${ }^{19}$

\subsubsection{Mechanical Instability}

Normally, when constructing an optical resonator, special vibration dampening tables and optical mounts are utilized. To perform CW-CRDS measurements on CHEWIE it was necessary to construct the ring-down cavity to be part of the plasma expansion chamber. Vibrations from the CHEWIE pumping system, pressure fluctuations in the chamber and thermal effects from the plasma all affected the effective cavity length and alignment of the ring-down cavity. Figure 4.18 shows the typical resonance peaks observed with the MARK-I 
CW-CRDS diagnostic.

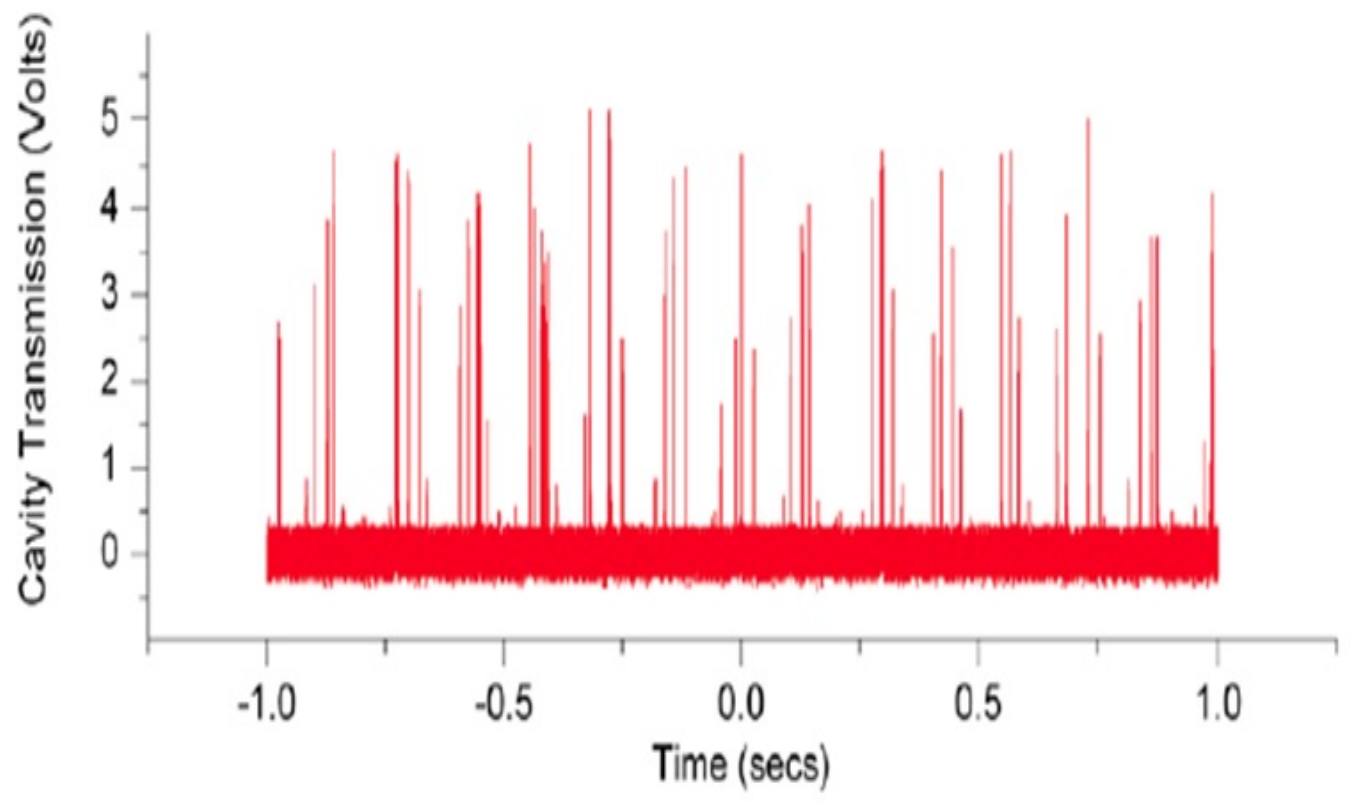

Figure 4.18: Typical resonance peaks in intensity versus acquisition time for the MARK-I CW-CRDS setup. Due to mechanical instability there is no clearly resolvable free spectral range. ${ }^{19}$

Excessive mechanical instabilities made it impossible to resolve the free spectral range (FSR) discussed in Section 4.12, making it exceedingly difficult to isolate only the $T E M_{00}$ modes. Mechanical instabilities occurred on time scales less than the time necessary for a resonance to reach its peak intensity in the cavity. This resulted in $T E M_{00}$ mode transmission intensities that could vary by up to $50 \%$ in magnitude. While the transmission intensity variations did not affect the ring-down time $\tau$ measurements, they prevented alignment optimization and the preferential selection of modes during data acquisition. 


\subsection{CW-CRDS Experimental Apparatus, MARK-II}

To address the issues identified in the MARK-I apparatus, a series of modifications were made to the CW-CRDS apparatus. A sketch of the MARK-II CW-CRDS experimental apparatus is shown in Figure 4.19. The TOPTICA diode laser and optical isolator were the same used in the MARK-I. To address the extinction ratio issues discussed in Section 4.6.2, a new AOM was purchased for the MARK-II experiments. The new IntraAction Corp. ATM-803DA1 AOM was capable of extinction ratios of $45 \mathrm{~dB}$. After the AOM two mirrors were used to "beam walk" the $m=1$ beam into a single mode fiber coupler. Using the single mode fiber serves a double purpose in the MARK-II setup. By transmitting the light through a single mode fiber, the diode laser and AOM are effectively decoupled from the CHEWIE plasma device. They may then be placed far away from the plasma source to mitigate the effects of vibrations and rf noise. The light emitted from a single mode fiber has a naturally Gaussian intensity profile, removing the need for a spatial filter. Indeed, the light from the single mode fiber had a more Gaussian profile than what was obtainable using the spatial filter. After the single mode fiber, the light was collimated and then mode matched to the optical cavity using a set of two mode matching lenses with $30 \mathrm{~cm}$ and $15 \mathrm{~cm}$ focal length lenses, respectively. The two mode matching lenses were separated by a distance of $49.5 \mathrm{~cm}$ with the $15 \mathrm{~cm}$ focal length lens being positioned $81.32 \mathrm{~cm}$ from the center of the ring-down cavity. Using two mode matching lenses permitted the use of a smaller beam waist at a known location. The two lens matching system provided better shaping of the injection beam and ultimately resulted in more efficient mode coupling. 




Figure 4.19: Schematic of the MARK-II CW-CRDS apparatus.

$I$ :Iris, $M$ :Beam steering mirror, $F C$ :Single mode fiber coupler, $L_{M 1}: f=30 \mathrm{~cm}$ mode matching lens, $L_{M 2}: f=15 \mathrm{~cm}$ mode matching lens, $H M$ : Highly reflective mirrors, AOM:Acoustooptic modulator

The MARK-II $82.55 \mathrm{~cm}$ ring-down cavity still consisted of two highly reflective mirrors, but no PZT was used to modulate the cavity length. With the level of mechanical instability inherent in the system, it was determined that there was sufficient intrinsic modulation of the cavity length to measure a full distribution. Eliminating the PZT had the added benefit of removing any chance the rf noise of the helicon source could couple into the PZT and its controller, thereby altering the cavity length. To further mitigate mechanical instabilities, the MARK-I vacuum mirror mounts were replaced by more compact and rigid commercial mounts from CRD-OPTICS ${ }^{\mathrm{TM}}$ (Figure 4.20). To maintain some distance between the plasma and highly reflective mirrors, $25 \mathrm{~cm}$ long Conflat ${ }^{\mathrm{TM}}$ nipples connected the mirror mounts to the CHEWIE expansion chamber. To address the particle deposition problem discussed in 
the previous section, each nipple was modified to include a gas feed port. When assembled to CHEWIE the nipple-mirror mount assemblies were separated from the plasma expansion chamber by a solid copper gasket with a $10 \mathrm{~mm}$ aperture along the optical axis of the cavity. During plasma measurements, argon gas is fed in through the nipples to create a gas curtain at the $10 \mathrm{~mm}$ aperture. These gas curtains successfully prevented particulates from depositing on the highly reflective mirrors and allowed for the MARK-II CW-CRDS setup to run for tens of hours without significant losses in reflectivity.
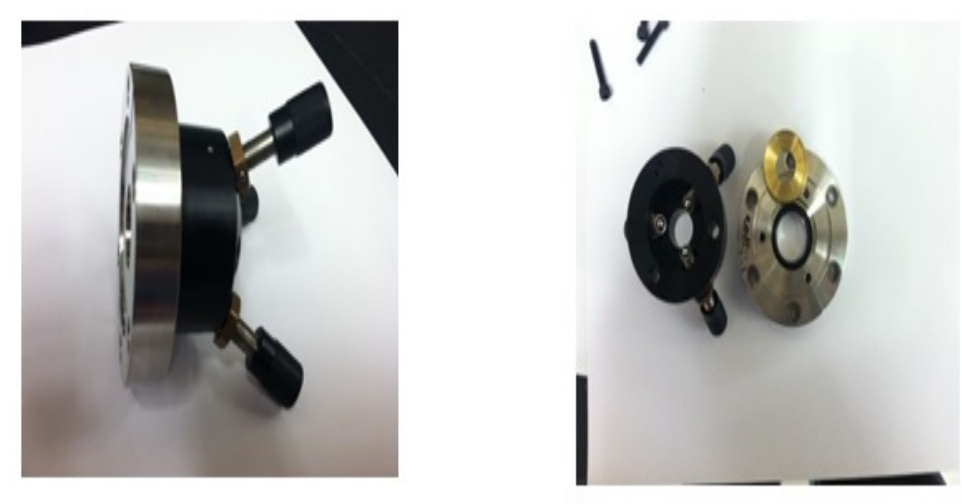

Figure 4.20: Commercial vacuum mirror mounts from CRD-OPTICS ${ }^{\mathrm{TM}}$. Shown above are an assembled mount (left) and the individual components (right).

The light emanating from the cavity was measured with the same New-Focus photoreceiver that had been used in the MARK-I experiment. Signals from the photo-receiver were sent to a GAGE MAGMA 16 bit digitizer and an improved tracking circuit. The GAGE digitizer used in the MARK-II has a higher vertical resolution and faster sample rate than the oscilloscope used in the MARK-I apparatus, resulting in more accurate measurements of the ring-down times. The functionality and purpose of the MARK-II tracking circuit remained the same as the MARK-I, but in-line buffer amps were integrated into the circuit to prevent power drain from the tracking circuit. The MARK-II acquisition scheme also underwent some 
technical changes. To increase acquisition rates custom software was written in LabWindows which allowed us to scan the laser, record wavelength measurements for measured ring-down events recorded by the GAGE digitizer and set the threshold voltage on the tracking circuit using a National Instruments BNC 2110 module. These modifications enabled us to acquire distributions over a large range of laser frequencies consisting of several hundred ring-downs in minutes. The overall error $\delta \tau / \tilde{\tau}$ of the MARK-II setup was $2 \%$ with no plasma.

\subsection{CW-CRDS MARK-II Data Analysis}

In CW-CRDS, the ring-down time $\tau$ is calculated by fitting an exponential function of the form

$$
f(t)=f_{0} \exp \left[\frac{-t}{\tau}\right]
$$

to the light transmitted by the cavity after resonance conditions have been met and the $m=1$ light has been extinguished. Figure 4.21 shows a single ring-down measurement. Here the red curve is the curve produced by fitting the data to Equation 4.22. 


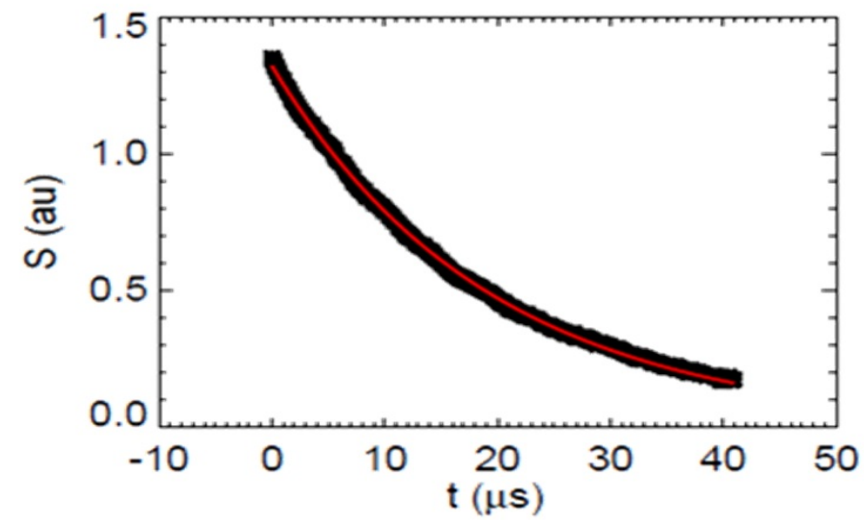

Figure 4.21: An example of a single ring-down measurement consisting of 8000 intensity measurements at a sample rate of $200 \mathrm{MS} / \mathrm{s}$. The red line is the curve calculated by fitting the data to an exponential decay.

The MARK-II data acquisition scheme enabled rapid measurements of a distribution of ring-down times around the rest frame absorption frequency of a plasma sample. Figure 4.22 shows a distribution of the calculated ring-downs times as a function of laser wavelength for each measurement. 


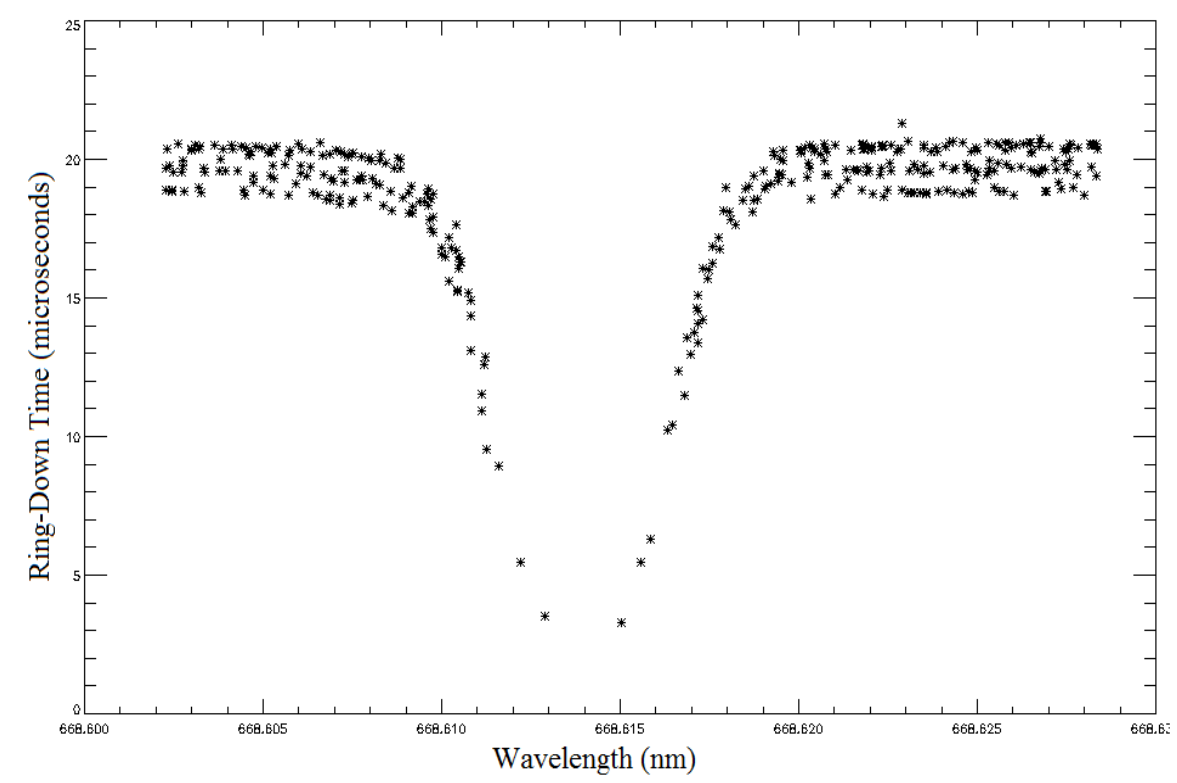

Figure 4.22: Distribution of ring-down measurements near the Ar II $668.6138 \mathrm{~nm}$ absorption line at $P=$ $6.2 \mathrm{mTorr}, R F=600 \mathrm{~W}, B=860 \mathrm{G}$ and Mass flow $=5$ SCCM.

It is clear from Figure 4.22 that there is a wavelength dependence in $\tau$. Near the rest frame absorption frequency, the ring-down times are significantly shorter. To determine the absolute line-integrated density of each measurement, each $\tau$ is compared to the average off resonance ring-down time $\tau_{0}$. The absolute-line integrated density in terms of the unitless absorption measure $A b s(\nu)$ is

$$
\operatorname{Abs}(\nu)=\int k(x, \nu) d x=\frac{d}{c}\left[\frac{1}{\tau_{\nu}}-\frac{1}{\tau_{0}}\right]
$$

where $d$ is the length of the plasma sample measured. In this work it was assumed the plasma occupied the whole of the expansion chamber, giving $d=15 \mathrm{~cm}$. Figure 4.23 shows the line integrated absorptions calculated for the ring-down times measured in Figure 4.22. Assuming the dominant broadening mechanism is Doppler broadening, Figure 4.23 is a measurement 
of the Doppler broadened absorption line. The distribution is fit to a Gaussian

$$
\operatorname{Abs}(\nu)=A b s\left(\nu_{0}\right) \exp \left[-\left(\nu-\nu_{0}\right)^{2} / \alpha_{s} T_{i}\right]
$$

where $\alpha_{s}$ is given by, $\alpha_{s}=2 e \nu_{\alpha} / m c^{2}$. For argon ions and neutrals respectively,

$$
\begin{aligned}
& \alpha_{A r I I}^{-1}=.092495 \mathrm{eV}(G H z)^{-2} \\
& \alpha_{A r I}^{-1}=.092384 \mathrm{eV}(G H z)^{-2} .
\end{aligned}
$$

In Figure 4.23 the red line shows the Gaussian fit to the absorption measurements and yields a temperature of $0.14 \mathrm{eV}$.

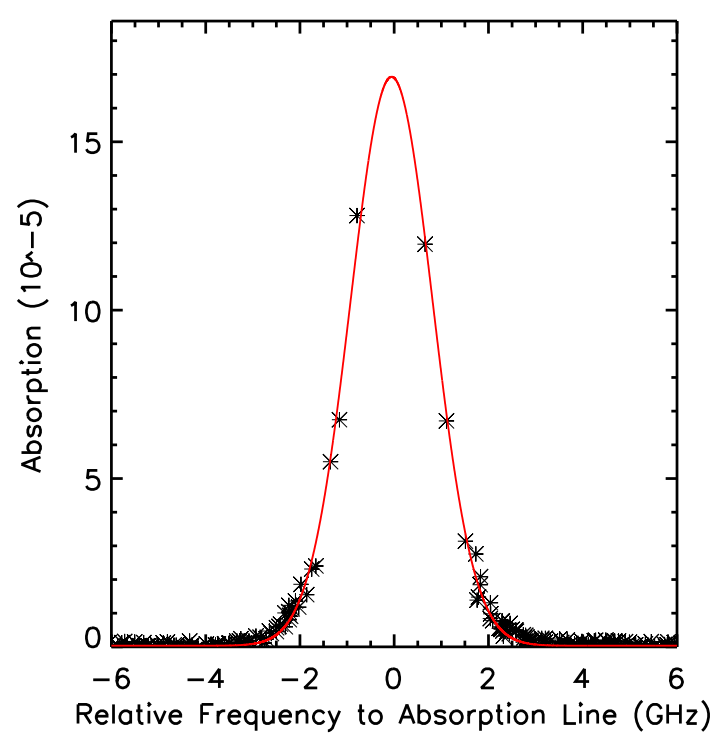

Figure 4.23: Doppler broadened absorption line of Ar II measured by the MARK-II CW-CRDS diagnostic at $P=6.2 \mathrm{mTorr}, R F=600 \mathrm{~W}, B=860 \mathrm{G}$ and Mass flow $=5$ SCCM..

The line integrated density is found by integrating Eqn.4.2 over physical space and fre- 
quency space to obtain

$$
\int n d x=8 \pi \frac{g_{1}}{g_{2}} \frac{\nu_{21}^{2}}{A_{21} c^{2}} \int A b s(\nu) d \nu
$$

where $n$ is the absolute density, $g$ is the level degeneracy of the state ( 1 being the lower state), $\nu$ is the transition frequency and $A$ is the Einstein coefficient. The values for the $\mathrm{Ar}$ II and Ar I transitions used in this work are shown in the table below. Using Eqn. 4.27 to calculate the absolute density of the distribution measured in Figure 4.23 yields an absolute density of $1.7 \times 10^{7} \mathrm{~cm}^{-3}$.

Table 4.1: Ar II and I parameters for density calculations

\begin{tabular}{|c|c|c|}
\hline Parameter & Ar II & Ar I \\
\hline$g_{1}$ & 8 & 3 \\
\hline$g_{2}$ & 6 & 1 \\
\hline$\nu_{21}$ & $4.483 \times 10^{1} 4(\mathrm{~Hz})$ & $4.488 \times 10^{1} 4(\mathrm{~Hz})$ \\
\hline$A_{21}$ & $1.07 \times 10^{7}\left(s^{-1}\right)$ & $2.36 \times 10^{5}\left(s^{-1}\right)$ \\
\hline
\end{tabular}




\section{Chapter 5}

\section{CW-CRDS and LIF}

The purpose of this work was to develop a new laser based optical diagnostic to resolve velocity distribution functions (vdfs) in a helicon plasma at parameters that are currently inaccessible with traditional LIF techniques. In previous chapters we discussed laser induced florescence, a powerful and mature optical diagnostic and the development and design of a new continuous wave cavity ring-down spectroscopy (CW-CRDS) diagnostic. To benchmark the performance of the new diagnostic, we compared measurements of VDFs obtained with LIF and CW-CRDS in the same plasmas. VDF measurements were obtained in helicon plasmas with varying rf powers (Section 5.1) and magnetic field strengths (Section 5.2). Also presented are CW-CRDS measurements at the plasma parameters noted in Chapter 3 for which Ar I VDF measurements were not possible with LIF.

There are a few caveats of which to be mindful in any comparison of LIF and CW-CRDS measurements. LIF is a spatially resolved technique, whereas CW-CRDS is inherently a line-integrated measurement. Temperature and density measurements obtained from CWCRDS represent the average temperature and density over the entire sample path length. The differences in the acquisition schemes for the two diagnostics also need to be considered. 
For the LIF measurements, each scan consisted of thirty-two evenly spaced data points in frequency space. For each data point, the fluorescence intensity was averaged over several seconds. For CW-CRDS, the laser is continuously swept across the absorption line width and ring-downs are recorded whenever resonance conditions are met in the cavity. The data points in each CW-CRDS measurement presented here are individual ring-down times, effectively snapshots of the plasma absorption on time scales of microseconds. Because of our inability to control when resonances occur, a measurement consisting of three hundred ringdowns might only include a small percentage of measurements within the Doppler broadened absorption line.

\subsection{RF power scan}

The first set of measurements were obtained by varying the rf power coupled into the plasma. Figures 5.1-5.5 show Ar II LIF and CW-CRDS measuremtents taken with rf powers that ranged from 300 watts to 700 watts in 100 watt increments. By using a wide range of input powers, we investigated helicon plasmas with different densities and temperatures. 

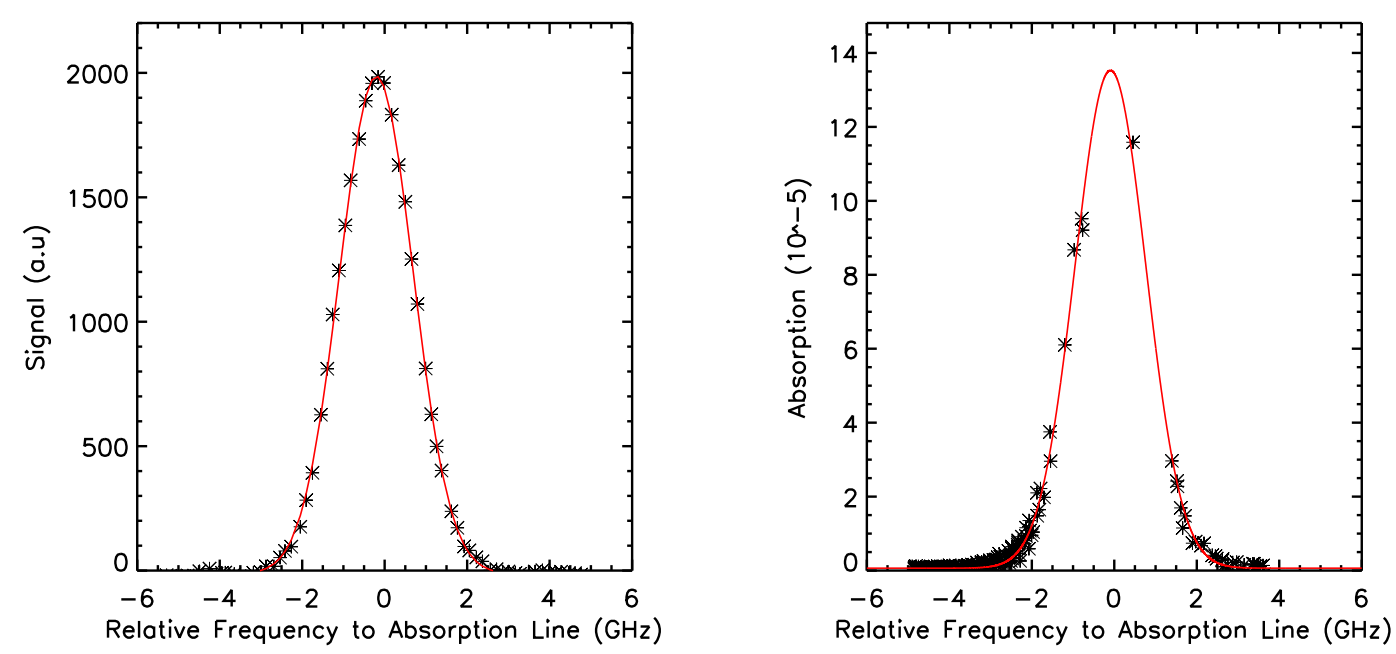

$\begin{array}{ll}\text { (a) } \mathrm{T}=.15 \pm .01(\mathrm{eV}) & \text { (b) } \mathrm{T}=.14 \pm .01(\mathrm{eV}), \mathrm{n}=1.3 \pm .1 \times 10^{7}\left(\mathrm{~cm}^{-3}\right)\end{array}$

Figure 5.1: Argon ion (a) LIF and (b) CW-CRDS VDFs measured at $r f=300 \mathrm{~W}, B=750 \mathrm{G}, P=8.3 \mathrm{mTorr}$, mass flow $=6$ SCCM.



(a) $\mathrm{T}=.18 \pm .01(\mathrm{eV})$

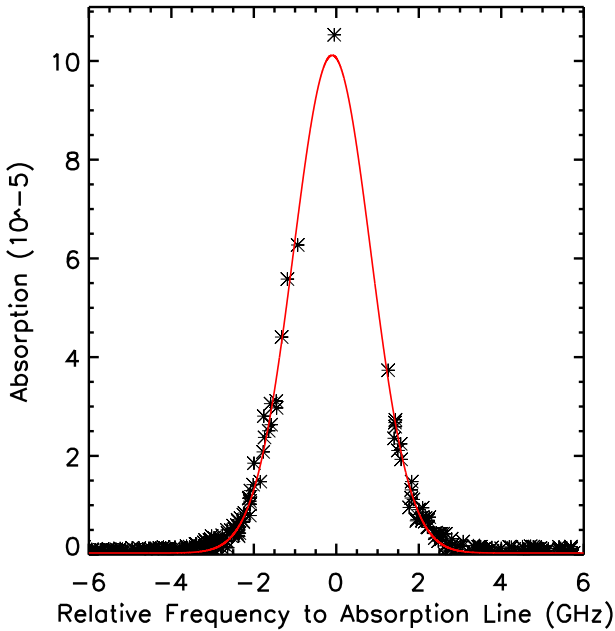

(b) $\mathrm{T}=.16 \pm .02(\mathrm{eV}), \mathrm{n}=1.1 \pm .1 \times 10^{7}\left(\mathrm{~cm}^{-3}\right)$

Figure 5.2: Argon ion (a) LIF and (b) CW-CRDS VDFs measured at $r f=400 \mathrm{~W}, B=750 \mathrm{G}, P=6.2$ mTorr, mass flow $=6$ SCCM. 


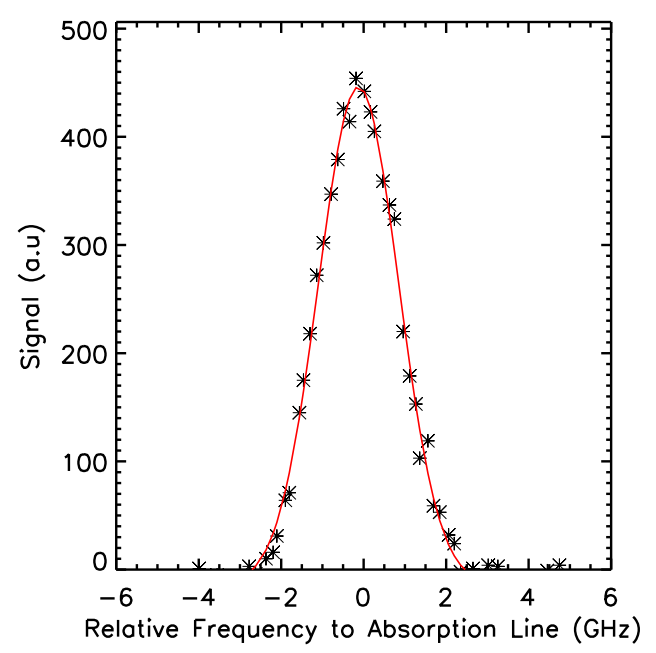

(a) $\mathrm{T}=.17 \pm .01(\mathrm{eV})$

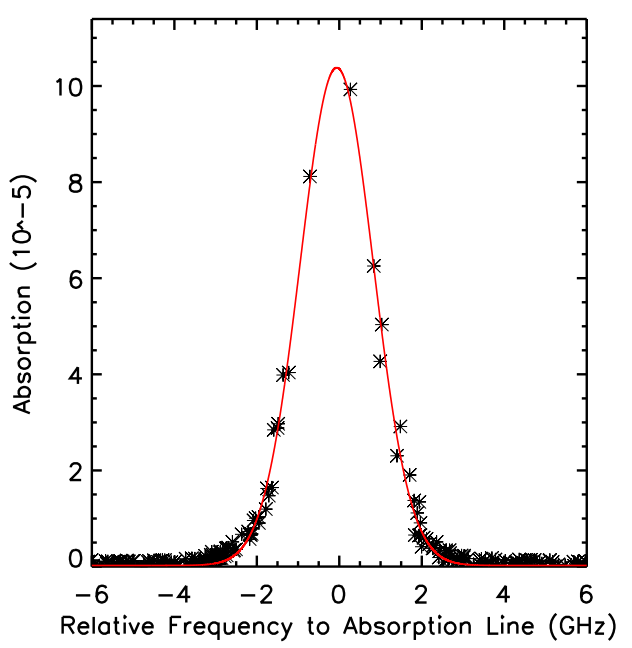

(b) $\mathrm{T}=.15 \pm .02(\mathrm{eV}), \mathrm{n}=1.1 \pm .1 \times 10^{7}\left(\mathrm{~cm}^{-3}\right)$

Figure 5.3: Argon ion (a) LIF and (b) CW-CRDS VDFs measured at $r f=500 \mathrm{~W}, B=750 \mathrm{G}, P=7 \mathrm{mTorr}$, mass flow $=6$ SCCM.

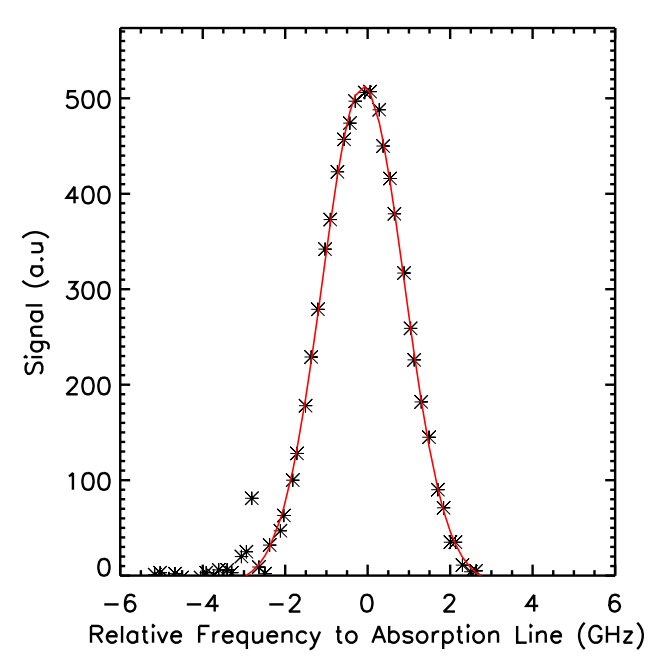

(a) $\mathrm{T}=.18 \pm .01(\mathrm{eV})$

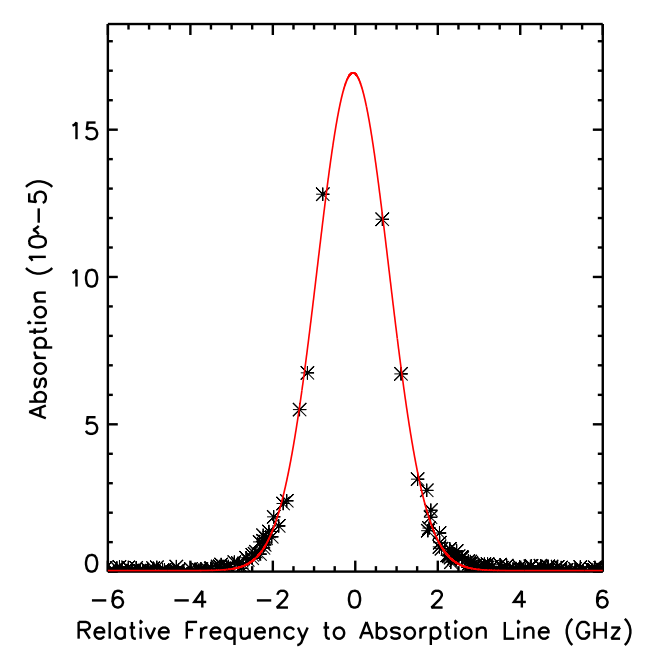

(b) $\mathrm{T}=.14 \pm .01(\mathrm{eV}), \mathrm{n}=1.7 \pm .2 \times 10^{7}\left(\mathrm{~cm}^{-3}\right)$

Figure 5.4: Argon ion (a) LIF and (b) CW-CRDS VDFs measured at $r f=600 \mathrm{~W}, B=750 \mathrm{G}, P=6.2 \mathrm{mTorr}$, mass flow $=6$ SCCM. 




(a) $\mathrm{T}=.20 \pm .01(\mathrm{eV})$

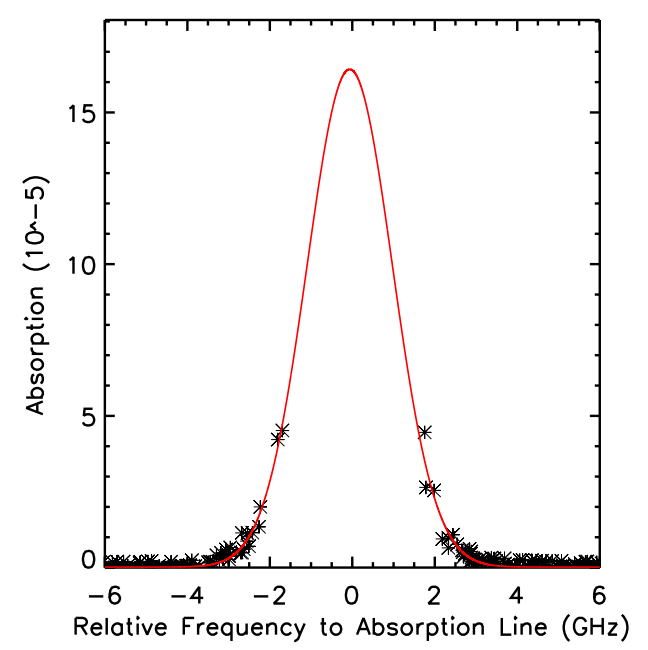

(b) $\mathrm{T}=.20 \pm .02(\mathrm{eV}), \mathrm{n}=2.0 \pm .2 \times 10^{7}\left(\mathrm{~cm}^{-3}\right)$

Figure 5.5: Argon ion (a) LIF and (b) CW-CRDS VDFs measured at $r f=700 \mathrm{~W}, B=750 \mathrm{G}, P=5.1 \mathrm{mTorr}$, mass flow $=6$ SCCM.

The ion temperatures calculated from the LIF measurements increase slightly with increasing rf power (Figure 5.6). The ion temperatures measured using CW-CRDS at 300, 400 and 700 Watts are very close to the ion temperatures calculated from LIF (Figure 5.6). There is some discrepancy in ion temperatures calculated using CW-CRDS and LIF at 500 and 600 Watts, with CW-CRDS yielding slightly lower ion temperatures than LIF at those rf power levels. It should also be noted that the metastable ion densities measured using CWCRDS also have no apparent proportionality with the rf power (Figure 5.7). The magnitude of metastable ion density, roughly $2 \times 10^{7} \mathrm{~cm}^{-3}$, is consistent with expectations for the density of an excited ion state in a plasma with a total ion density on the order of $1 \times 10^{11} \mathrm{~cm}^{-3}$. 


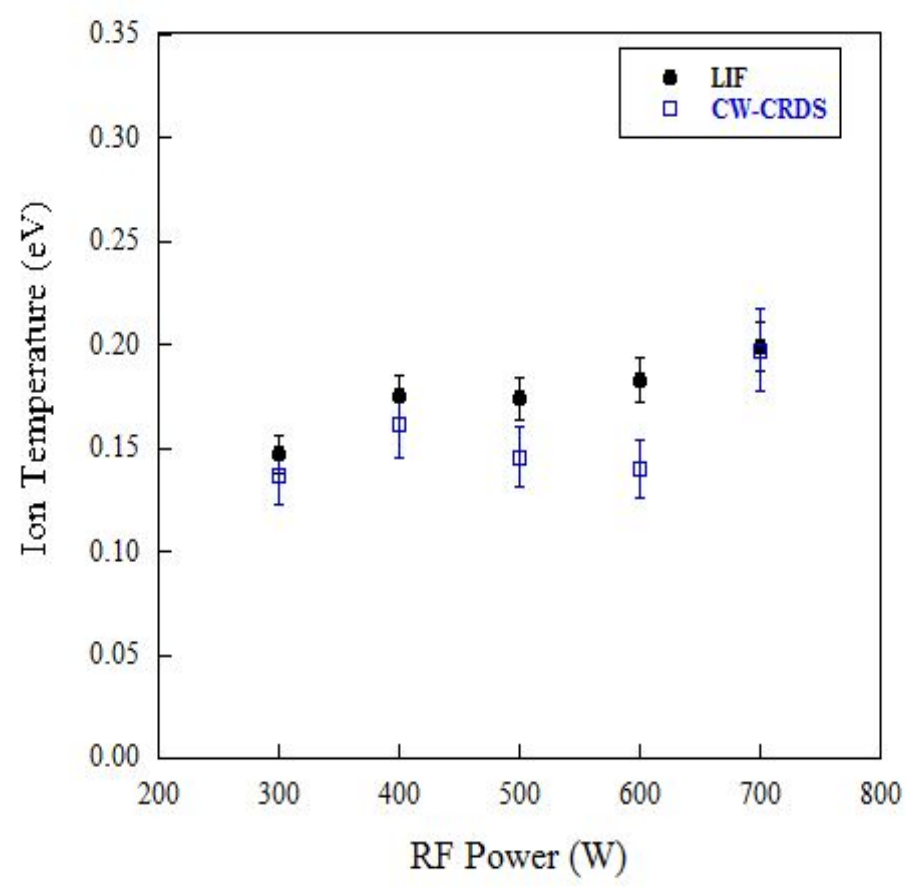

Figure 5.6: Plasma ion temperatures calculated from LIF and CW-CRDS measurements for different rf powers. 


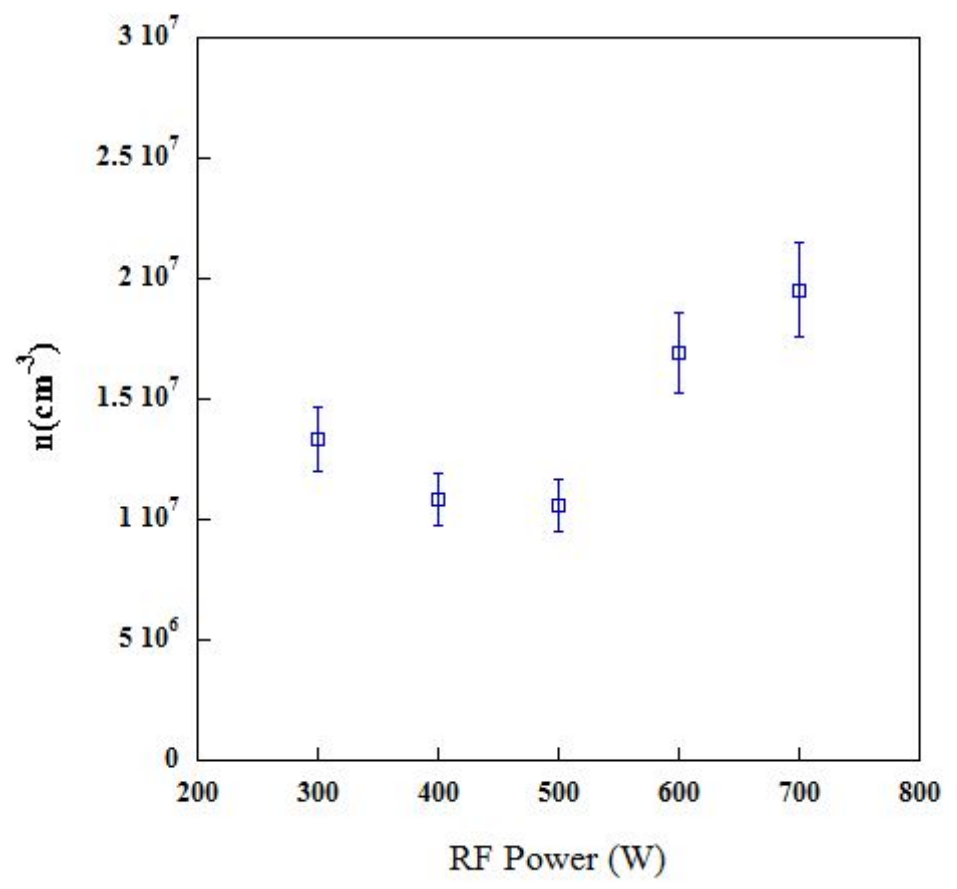

Figure 5.7: Metastable ion density calculated from CW-CRDS measurements for different rf powers.

\subsection{Magnetic Field Scan}

The next comparisons were all made at 500 Watts of rf power, but the magnetic field strength was varied from $630 \mathrm{G}$ to $860 \mathrm{G}$. Helicon sources have a well-known scaling of density and ion temperature with magnetic field strength. Shown in Figures 5.8-5.12 are Ar II VDF measurements made using both diagnostic techniques for each magnetic field strength. 

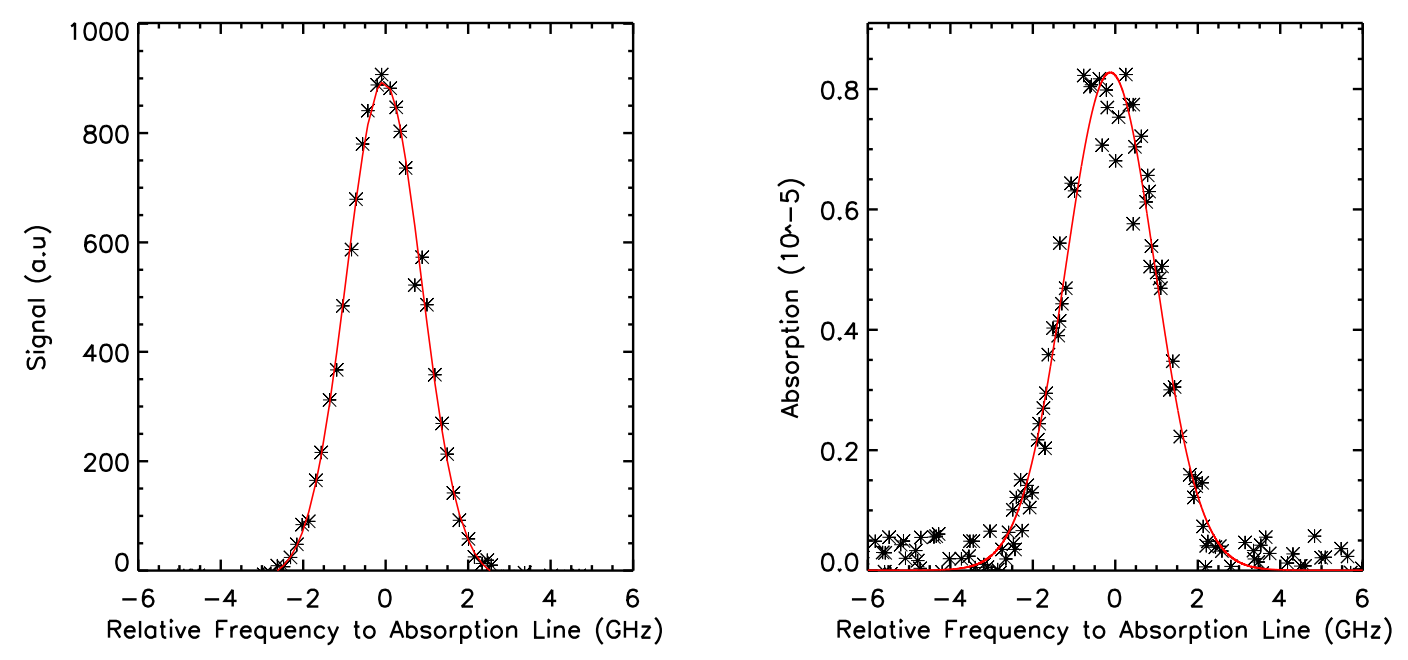
(a) $\mathrm{T}=.15 \pm .01(\mathrm{eV})$
(b) $\mathrm{T}=.22 \pm .02(\mathrm{eV}), \mathrm{n}=1.0 \pm .1 \times 10^{6}\left(\mathrm{~cm}^{-3}\right)$

Figure 5.8: Argon (a) LIF and (b) CW-CRDS VDFs measured at $r f=500 \mathrm{~W}, B=630 \mathrm{G}, P=8.4$ mTorr, mass flow $=6$ SCCM.



(a) $\mathrm{T}=.15 \pm .01(\mathrm{eV})$

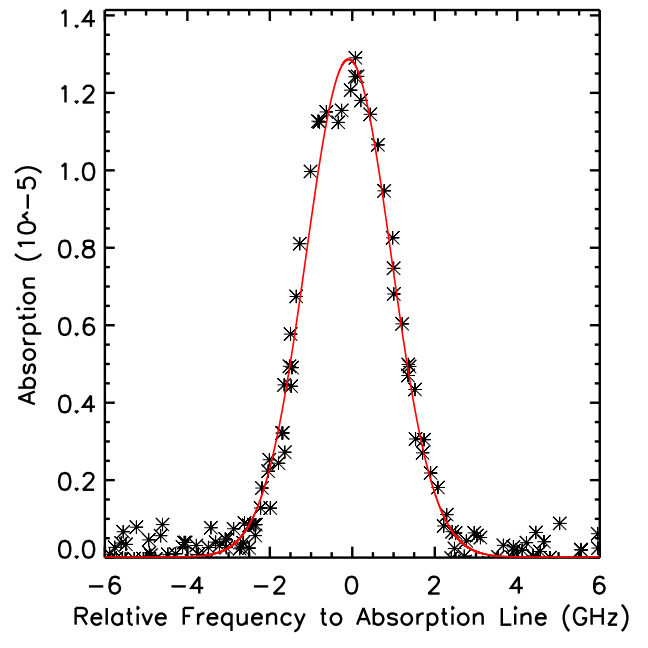

(b) $\mathrm{T}=.20 \pm .02(\mathrm{eV}), \mathrm{n}=1.5 \pm .2 \times 10^{6}\left(\mathrm{~cm}^{-3}\right)$

Figure 5.9: Argon (a) LIF and (b) CW-CRDS VDFs measured at $r f=500 \mathrm{~W}, B=690 \mathrm{G}, P=6.4$ mTorr, mass flow $=6$ SCCM. 


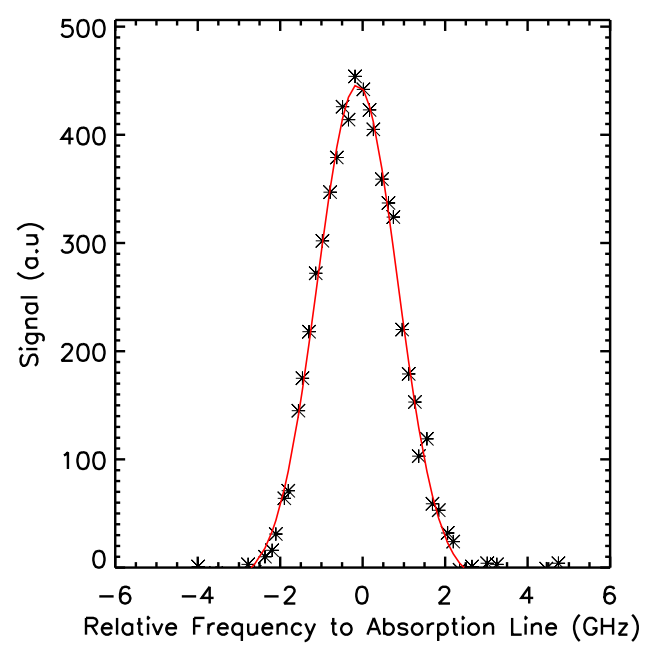

(a) $\mathrm{T}=.17 \pm .01(\mathrm{eV})$

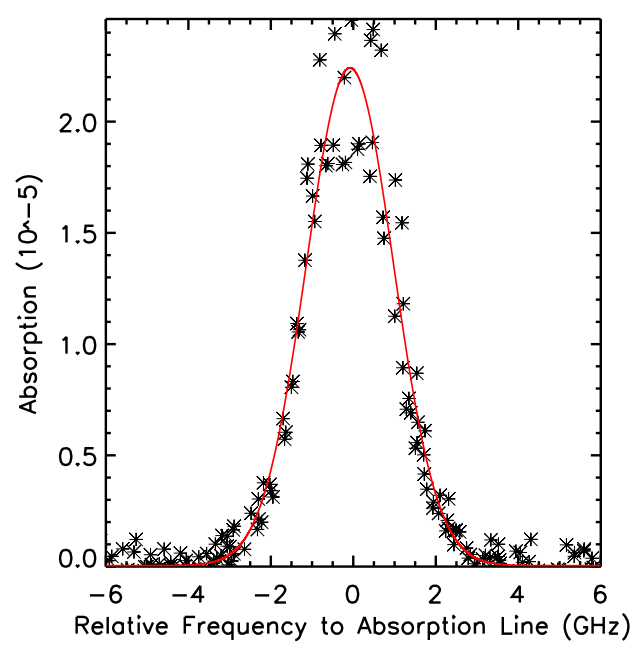

(b) $\mathrm{T}=.21 \pm .02(\mathrm{eV}), \mathrm{n}=2.7 \pm .3 \times 10^{6}\left(\mathrm{~cm}^{-3}\right)$

Figure 5.10: Argon (a) LIF and (b) CW-CRDS VDFs measured at $r f=500 \mathrm{~W}, B=750 \mathrm{G}, P=7$ mTorr, mass flow $=6$ SCCM.

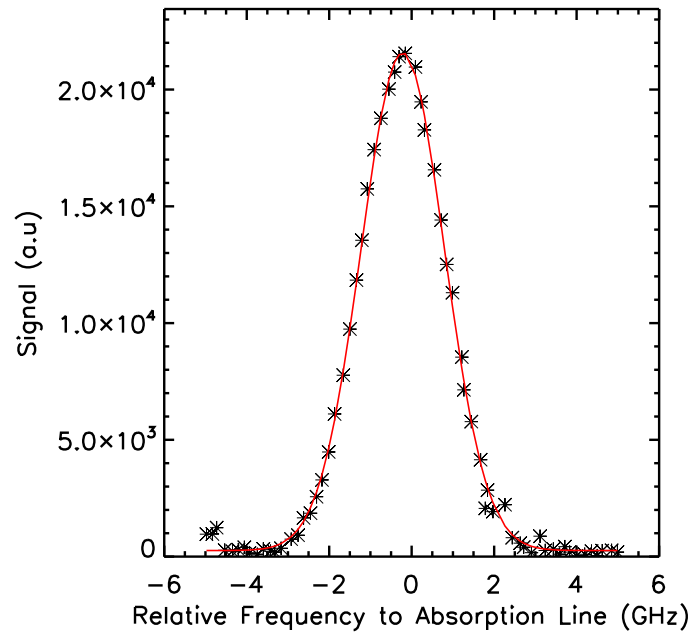

(a) $\mathrm{T}=.18 \pm .01(\mathrm{eV})$

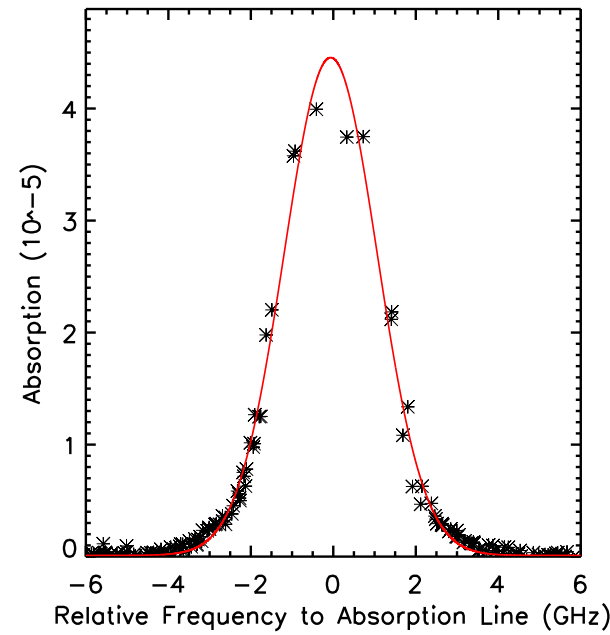

(b) $\mathrm{T}=.24 \pm .02(\mathrm{eV}), \mathrm{n}=5.8 \pm .6 \times 10^{6}\left(\mathrm{~cm}^{-3}\right)$

Figure 5.11: Argon (a) LIF and (b) CW-CRDS VDFs measured at $r f=500 \mathrm{~W}, B=800 \mathrm{G}, P=4.6$ mTorr, mass flow $=6$ SCCM. 


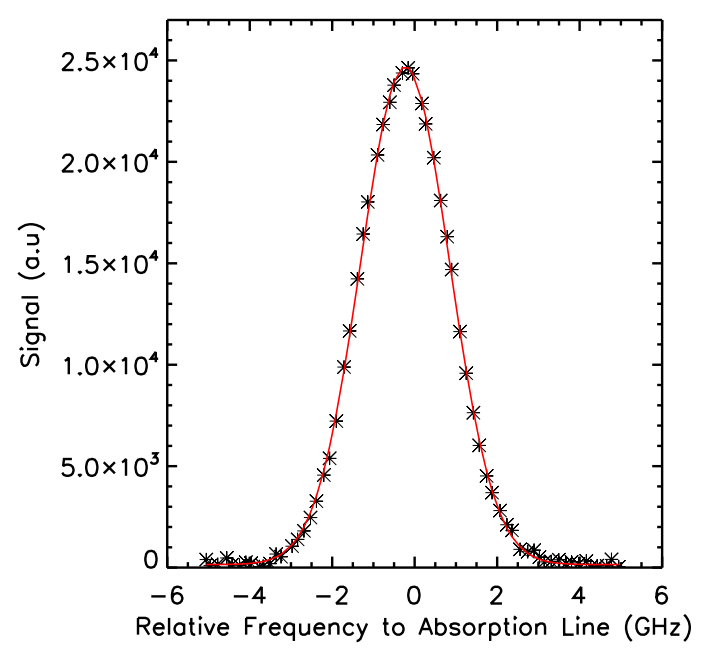

(a) $\mathrm{T}=.21 \pm .01(\mathrm{eV})$



(b) $\mathrm{T}=.22 \pm .02(\mathrm{eV}), \mathrm{n}=8.7 \pm .9 \times 10^{6}\left(\mathrm{~cm}^{-3}\right)$

Figure 5.12: Argon (a) LIF and (b) CW-CRDS VDFs measured at $r f=500 \mathrm{~W}, B=860 \mathrm{G}, P=3.8 \mathrm{mTorr}$, mass flow $=6$ SCCM.

The LIF measurements show an increasing ion temperature and density with increasing magnetic field strength as expected for a helicon plasma source (Figures 5.13 and 5.14). ${ }^{28}$ Discrepancies between the temperatures measured using LIF and CW-CRDS are shown in Figure 5.13, where ion temperatures obtained with the CW-CRDS diagnostic are consistently higher. Note that for the ion temperature measurements shown in Figure 5.13 the two diagnostics disagreed most at the smallest metastable ion density measured by CW-CRDS and agreed the most closely for the largest metastable ion density measurements. 


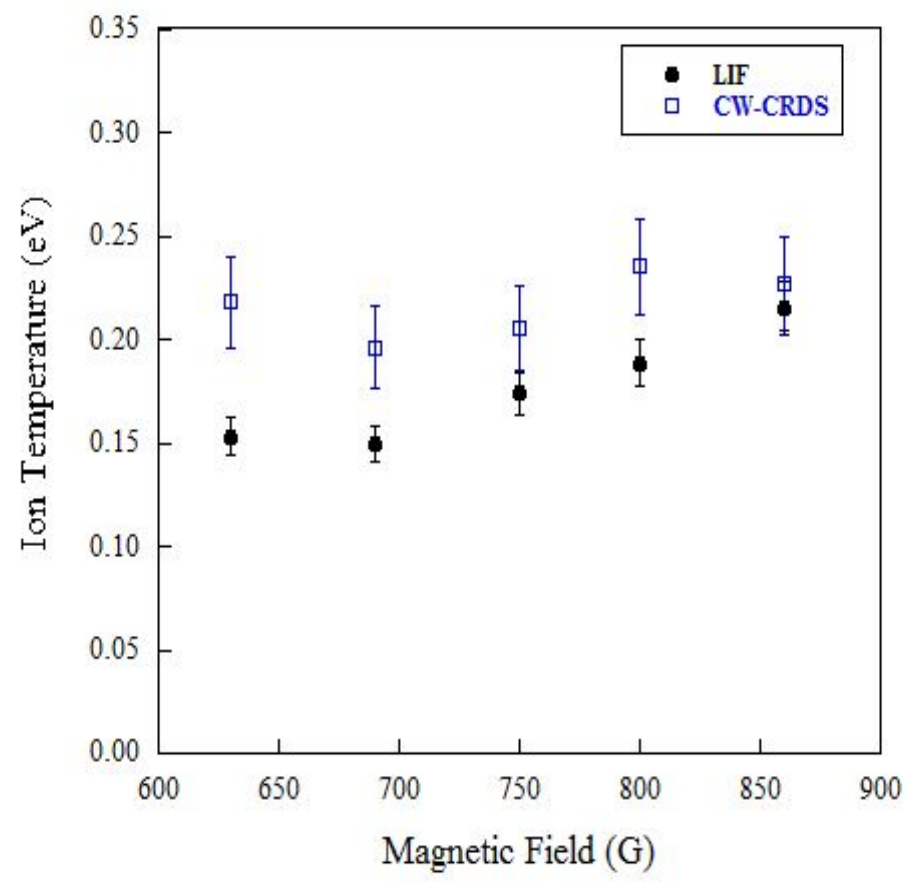

Figure 5.13: Plasma ion temperatures calculated from LIF and CW-CRDS measurements for different magnetic field strengths. 


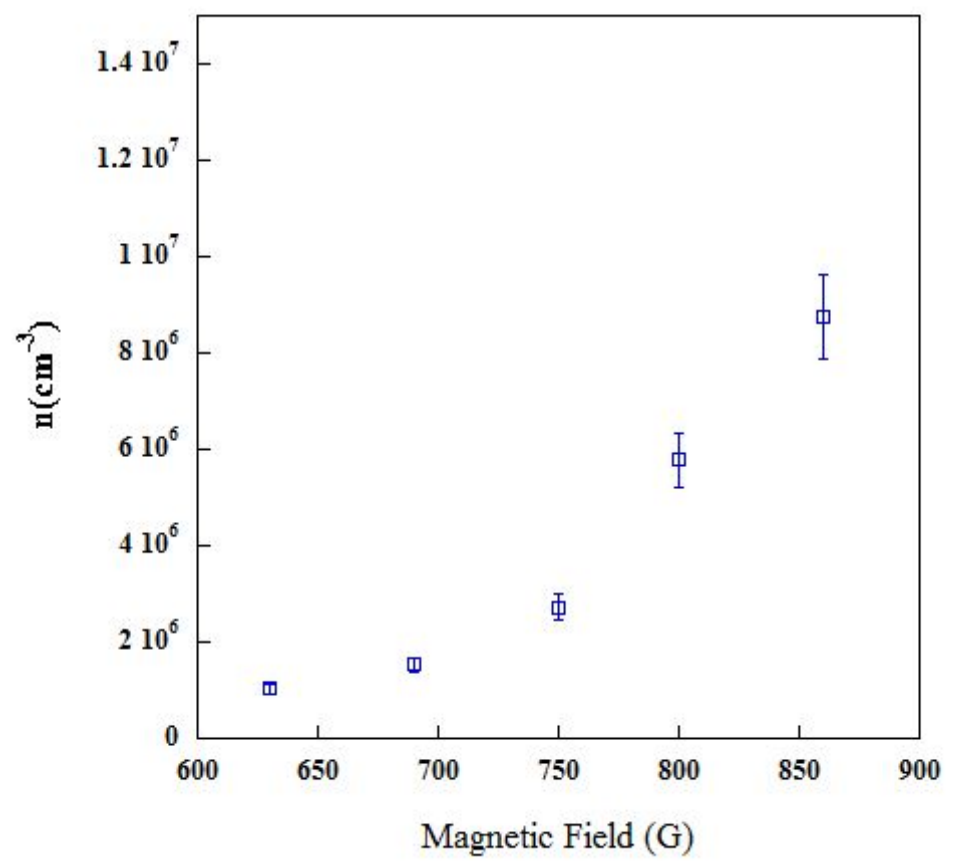

Figure 5.14: Metastable ion density calculated from CW-CRDS measurements versus magnetic field strengths.

\subsection{Ar I and Ar II measurements}

As described in Chapter 3, LIF at 667 is unable to resolve the Doppler broadened absorption line of Ar I for many typical helicon source parameters. The Ar I and Ar II measurements presented here, in sections 5.3.2 and 5.3.1 respectively, were obtained at 300 Watts rf power and magnetic field strengths that varied from 630 G to 860 G. At such small rf powers, the plasma is weakly ionized and the density of the initial metastable state is quite small. Figure 5.15(a) shows that the ion metastable density is only $5 \times 10^{5} \mathrm{~cm}^{-3}$. The CW-CRDS measurements are inconsistent and the absorption line is unusually broad. At slightly larger 
magnetic fields [Figure 5.16(b)] the CW-CRDS measurements are again quite irreproducible near the peak of the absorption line.

\subsubsection{Ar II measurements}

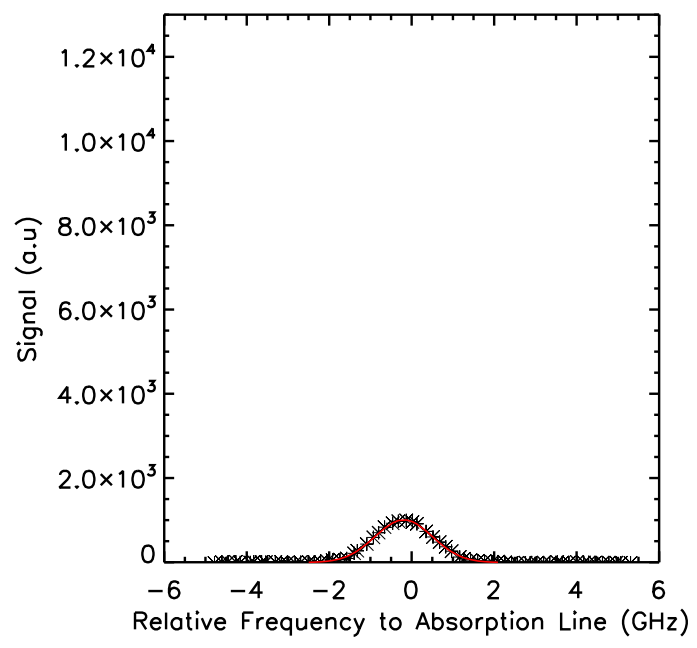

(a) $\mathrm{T}=.09 \pm .01(\mathrm{eV})$

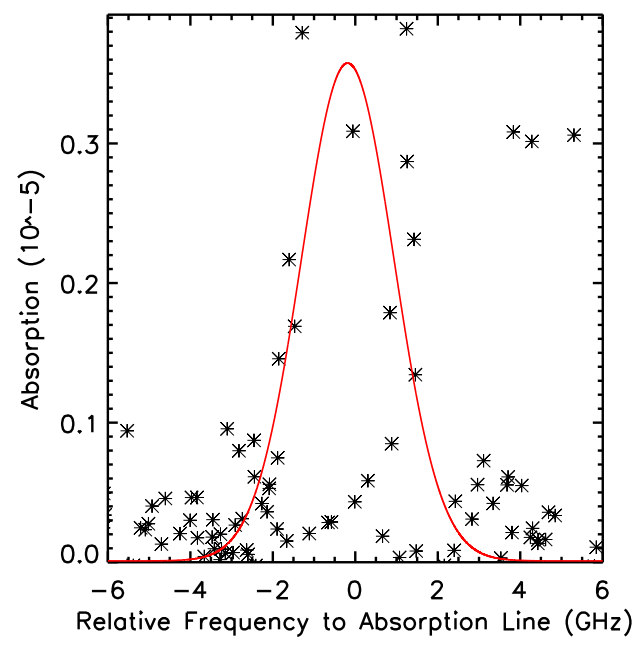

(b) $\mathrm{T}=.23 \pm .05(\mathrm{eV}), \mathrm{n}=4.6 \pm 1.1 \times 10^{5}\left(\mathrm{~cm}^{-3}\right)$

Figure 5.15: Argon ion (a) LIF and (b) CW-CRDS VDFs measured at $r f=300 \mathrm{~W}, B=630 \mathrm{G}, P=12.5$ mTorr, mass flow $=5$ SCCM. 

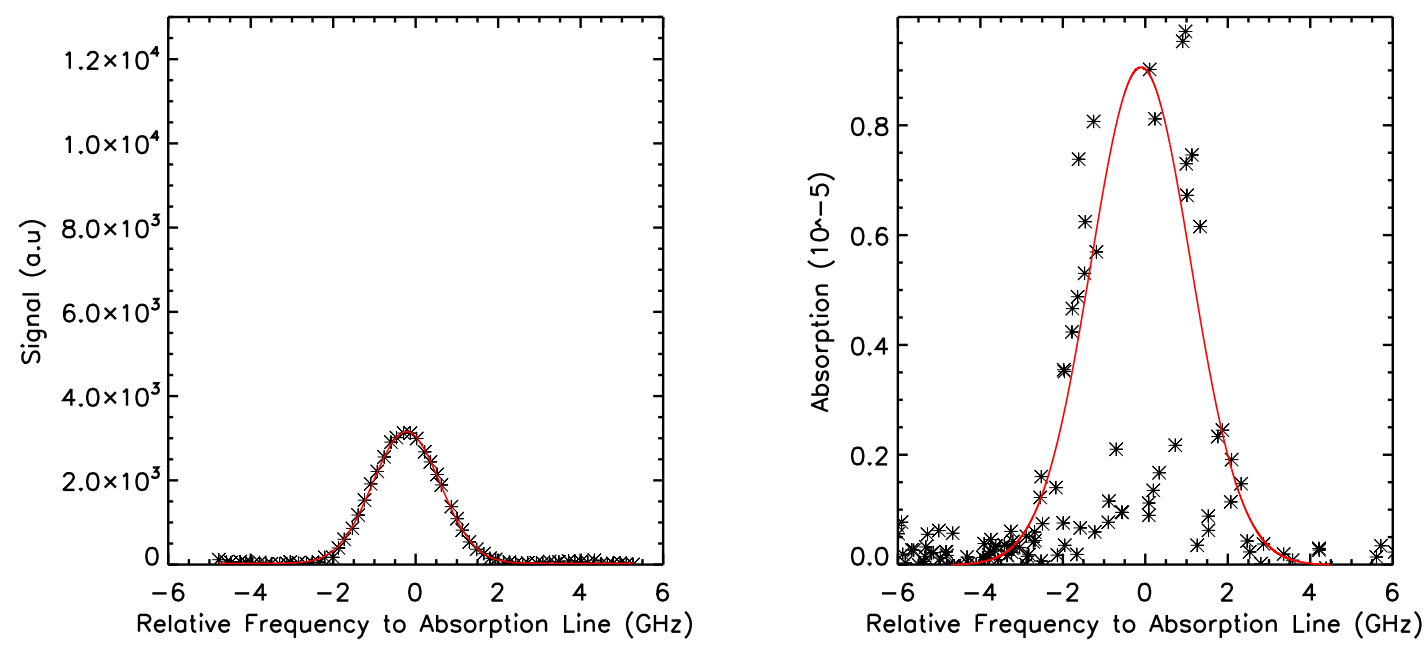
(a) $\mathrm{T}=.12 \pm .01(\mathrm{eV})$
(b) $\mathrm{T}=.27 \pm .04(\mathrm{eV}), \mathrm{n}=1.3 \pm .2 \times 10^{5}\left(\mathrm{~cm}^{-3}\right)$

Figure 5.16: Argon ion (a) LIF and (b) CW-CRDS VDFs measured at $r f=300 \mathrm{~W}, B=690 \mathrm{G}, P=9.8$ $\mathrm{mTorr}$, mass flow $=5 \mathrm{SCCM}$.

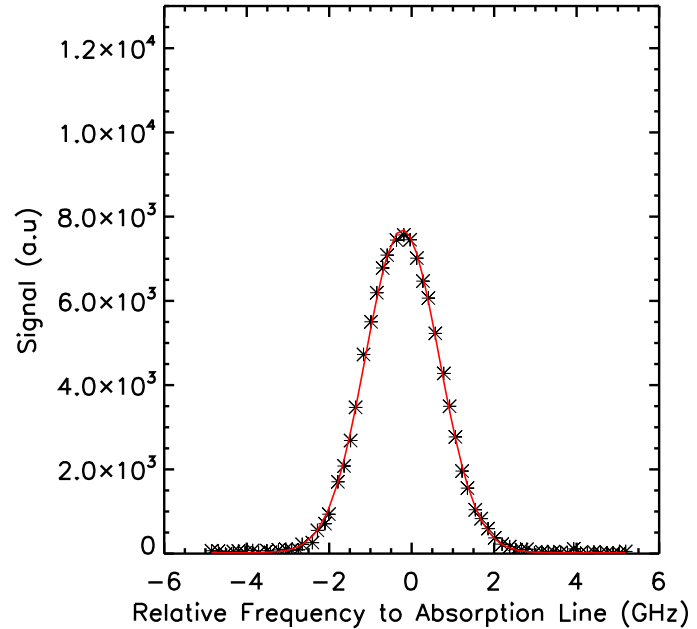

(a) $\mathrm{T}=.15 \pm .01(\mathrm{eV})$



(b) $\mathrm{T}=.21 \pm .02(\mathrm{eV}), \mathrm{n}=2.6 \pm .3 \times 10^{5}\left(\mathrm{~cm}^{-3}\right)$

Figure 5.17: Argon ion (a) LIF and (b) CW-CRDS VDFs measured at $r f=300 \mathrm{~W}, B=750 \mathrm{G}, P=7.8$ mTorr, mass flow $=5$ SCCM. 


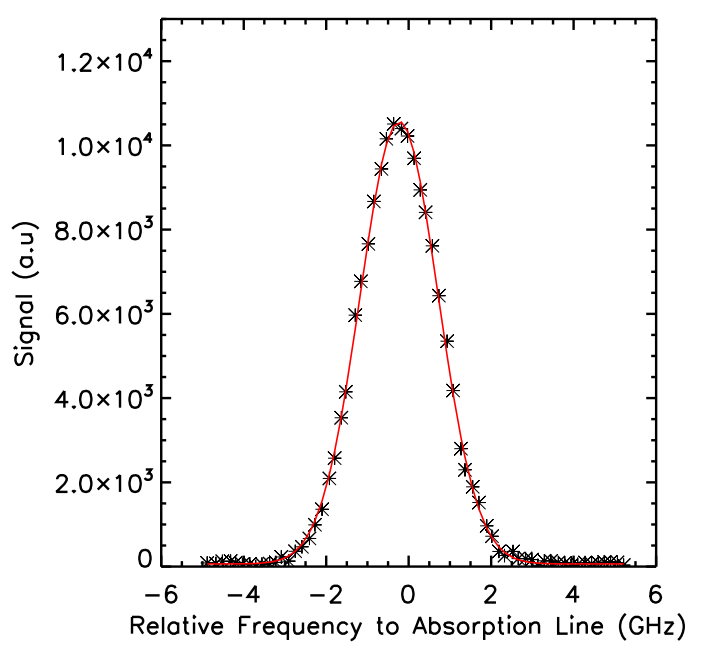

(a) $\mathrm{T}=.16 \pm .01(\mathrm{eV})$



(b) $\mathrm{T}=.18 \pm .02(\mathrm{eV}), \mathrm{n}=4.5 \pm .5 \times 10^{5}\left(\mathrm{~cm}^{-3}\right)$

Figure 5.18: Argon ion (a) LIF and (b) CW-CRDS VDFs measured at $r f=300 \mathrm{~W}, B=800 \mathrm{G}, P=6.8$ $\mathrm{mTorr}$, mass flow $=5 \mathrm{SCCM}$.

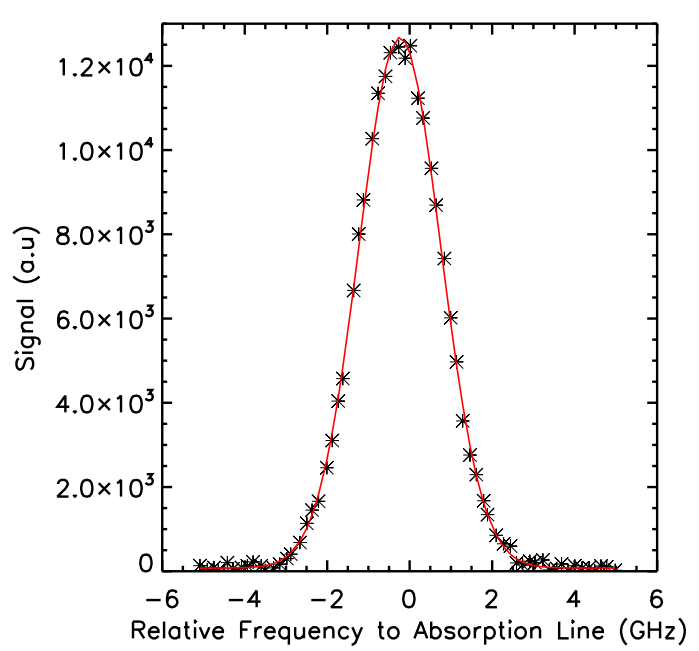

(a) $\mathrm{T}=.18 \pm .01(\mathrm{eV})$

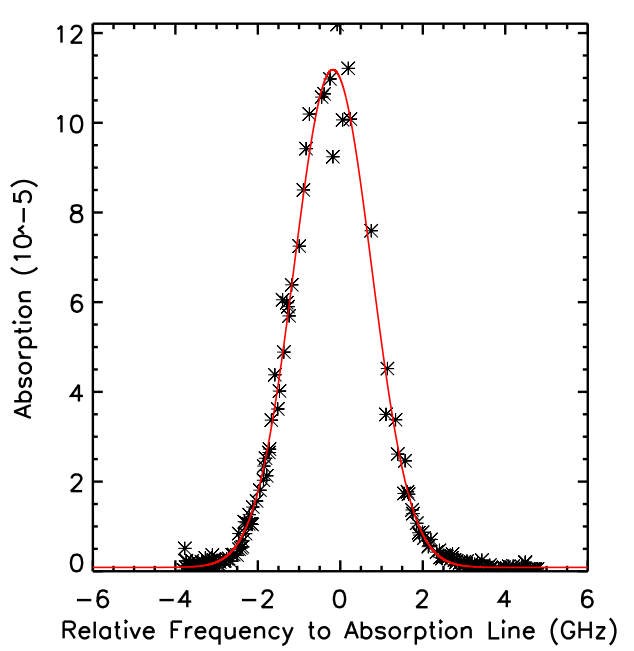

(b) $\mathrm{T}=.16 \pm .02(\mathrm{eV}), \mathrm{n}=12 \pm 1.2 \times 10^{5}\left(\mathrm{~cm}^{-3}\right)$

Figure 5.19: Argon ion (a) LIF and (b) CW-CRDS VDFs measured at $r f=300 \mathrm{~W}, B=860 \mathrm{G}, P=6.4$ mTorr, mass flow $=5$ SCCM.

The measurements shown in Figures 5.15 -5.19 are different from the Ar II measurements discussed in the previous section. Special care was taken with the LIF diagnostic so that the relative densities of Ar II could be compared by looking at the amplitude of the scans. This 
was accomplished by keeping the LIF acquisition setting constant throughout the experiment. For the CW-CRDS measurements, particularly Figures 5.15(b) and 5.16(b), it is important to remember that each data point is essentially a snapshot of the plasma. It was found that it was hard to couple rf power into the plasma for low rf powers and low magnetic field strengths. This caused the plasma to "flicker" and it is hypothesized that the ringdown times here are accurate for the instant they were taken. When the magnetic field strength was increased the rf coupling became more efficient and the flickering became less pronounced in the CW-CRDS measurements. These flickers are not as apparent in the LIF measurements because each data point is the result of several seconds of averaging.

Figurs 5.20 shows the effects of magnetic field strength on ion temperature measurements made with each diagnostic. For LIF measurements, the calculated temperature increased with increasing the magnetic field. This is similar to the results of Section 5.2. However, the temperatures calculated from CW-CRDS measurements are wildly inaccurate for the smaller magnetic field strengths and it is only once the plasma density increases that the quality of the CW-CRDS data improve. The CW-CRDS measurements at the larger magnetic fields are in excellent agreement with the LIF measurements.

Shown in Figure 5.21 are the metastable ion densities obtained from the LIF measurements (arb. units) and the CW-CRDS measurements. Since both LIF and CW-CRDS probe the same initial metastable ion state, it is significant that both techniques show similar scaling of the metastable state density with magnetic field strength. 


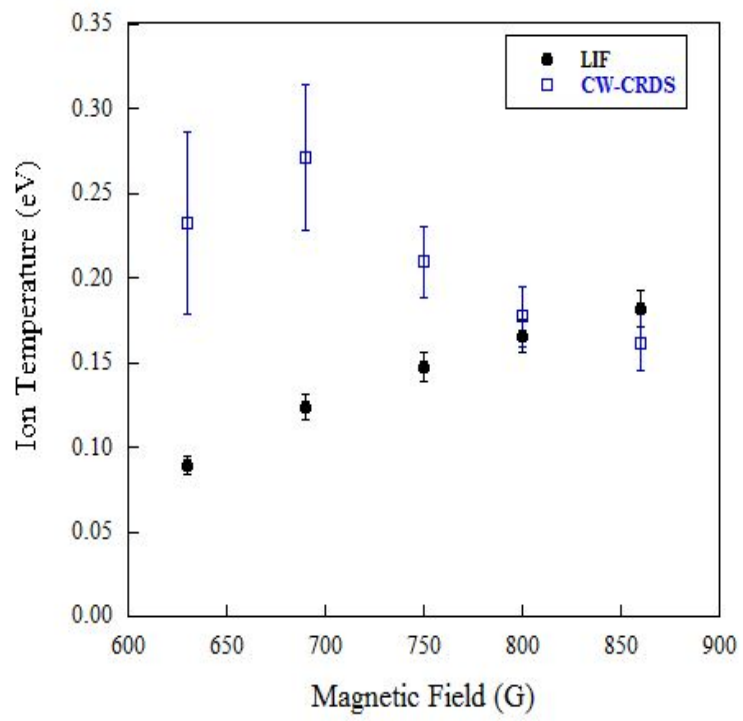

Figure 5.20: Ar II temperatures calculated from LIF and CW-CRDS measurements for different magnetic field strengths. 


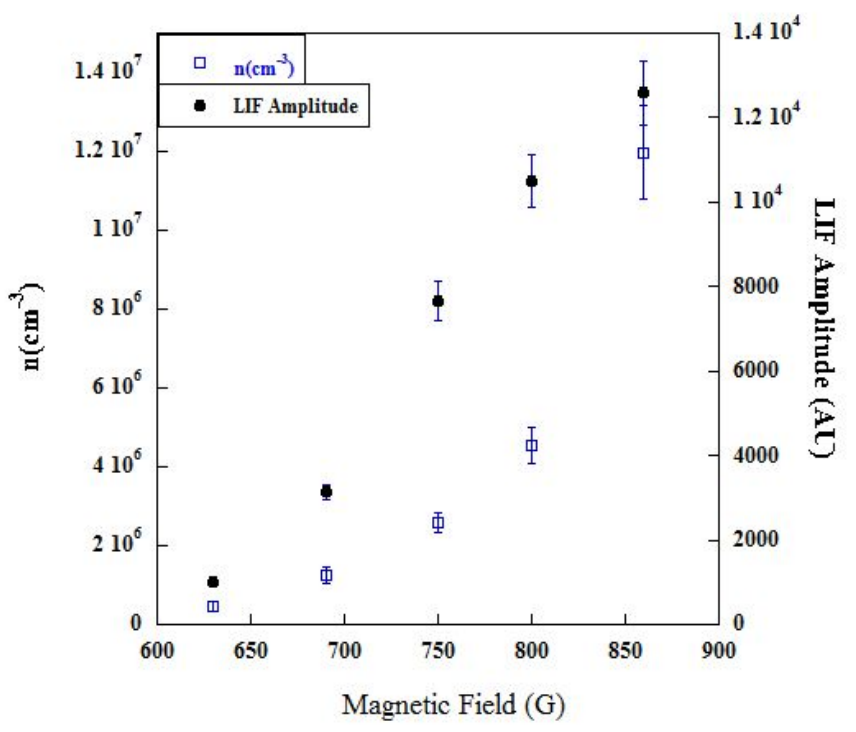

Figure 5.21: Ar II density $n$ calculated with CW-CRDS measurements and LIF VDF amplitudes for different magnetic field strengths. 


\subsubsection{Ar I measurements}

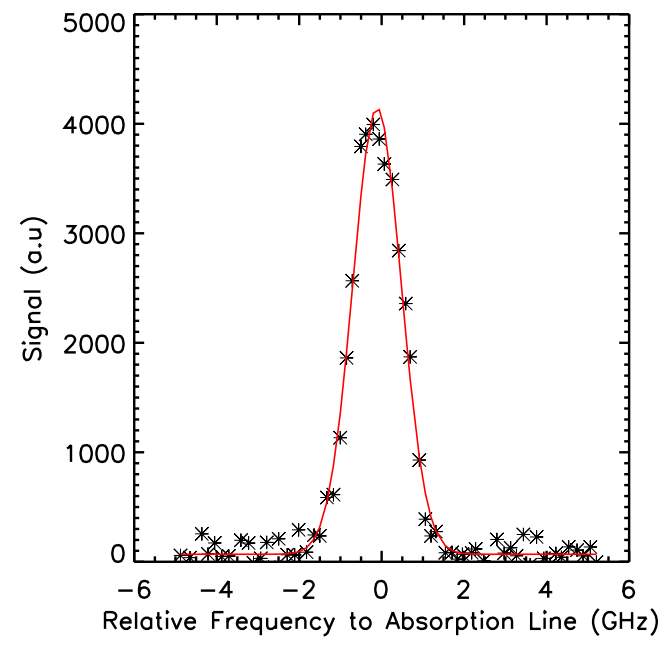

(a) $\mathrm{T}=.06 \pm .001(\mathrm{eV})$



(b) $\mathrm{T}=.12 \pm .01(\mathrm{eV}), \mathrm{n}=1.9 \pm .2 \times 10^{8}\left(\mathrm{~cm}^{-3}\right)$

Figure 5.22: Argon neutral (a) LIF and (b) CW-CRDS VDFs measured at $r f=300 \mathrm{~W}, B=860 \mathrm{G}, P=6.3$ mTorr, mass flow $=5$ SCCM.

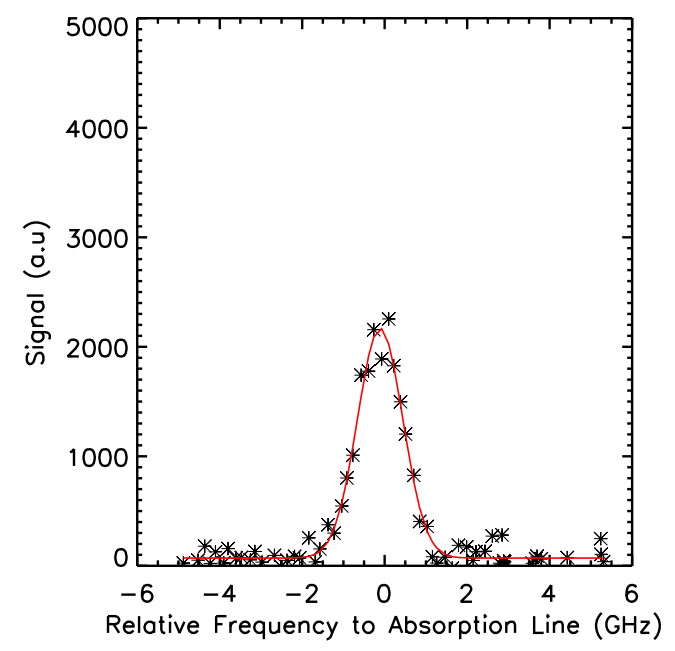

(a) $\mathrm{T}=.05 \pm .001(\mathrm{eV})$



(b) $\mathrm{T}=.11 \pm .01(\mathrm{eV}), \mathrm{n}=2.2 \pm .2 \times 10^{8}\left(\mathrm{~cm}^{-3}\right)$

Figure 5.23: Argon neutral (a) LIF and (b) CW-CRDS VDFs measured at $r f=300 \mathrm{~W}, B=800 \mathrm{G}, P=6.8$ mTorr, mass flow $=5$ SCCM. 


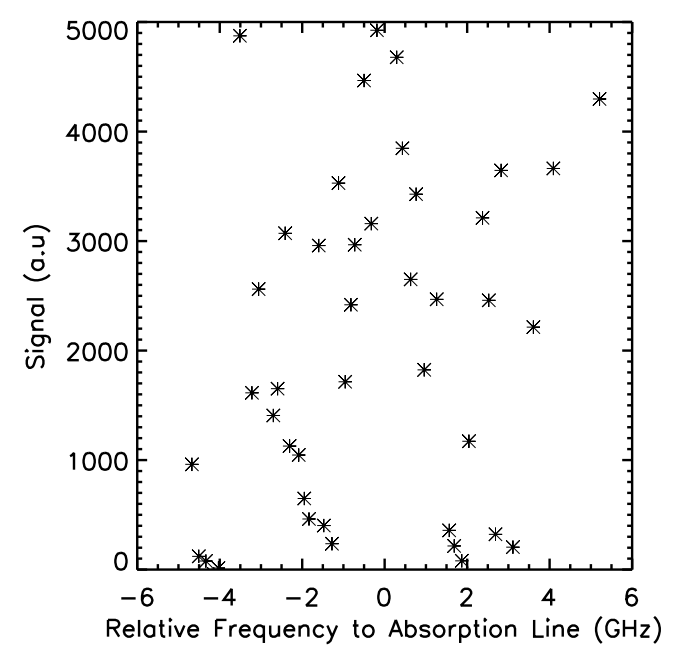

(a) $\mathrm{T}$ is unattainable

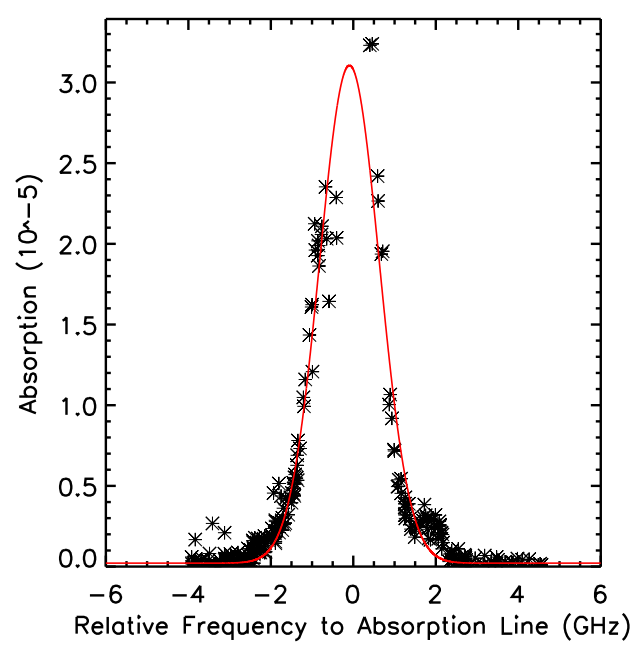

(b) $\mathrm{T}=.10 \pm .01(\mathrm{eV}), \mathrm{n}=2.6 \pm .3 \times 10^{8}\left(\mathrm{~cm}^{-3}\right)$

Figure 5.24: Argon neutral (a) LIF and (b) CW-CRDS VDFs measured at $r f=300 \mathrm{~W}, B=750 \mathrm{G}, P=7.6$ mTorr, mass flow $=5$ SCCM.

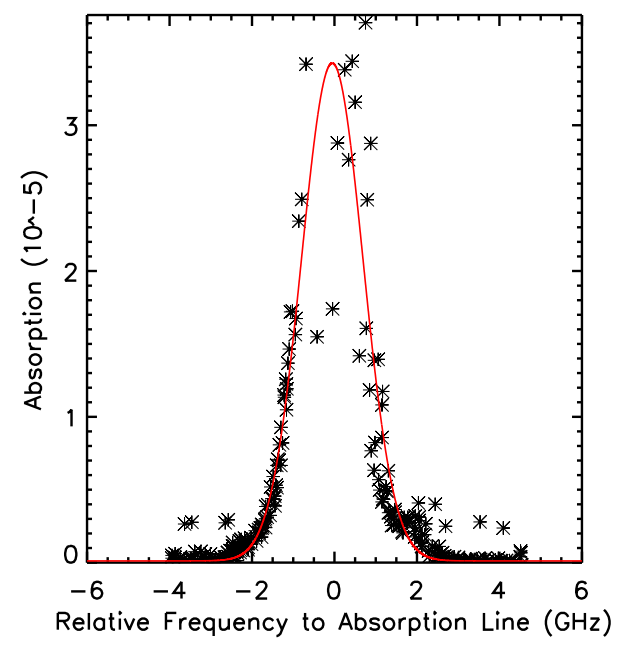

Figure 5.25: (a) Argon neutral CW-CRDS VDF measured at $r f=300 \mathrm{~W}, B=690 \mathrm{G}, P=9.7$ mTorr, mass flow $=5$ SCCM; T $=.10 \pm .01(\mathrm{eV}), \mathrm{n}=3.0 \pm .3 \times 10^{8}\left(\mathrm{~cm}^{-3}\right)$. 


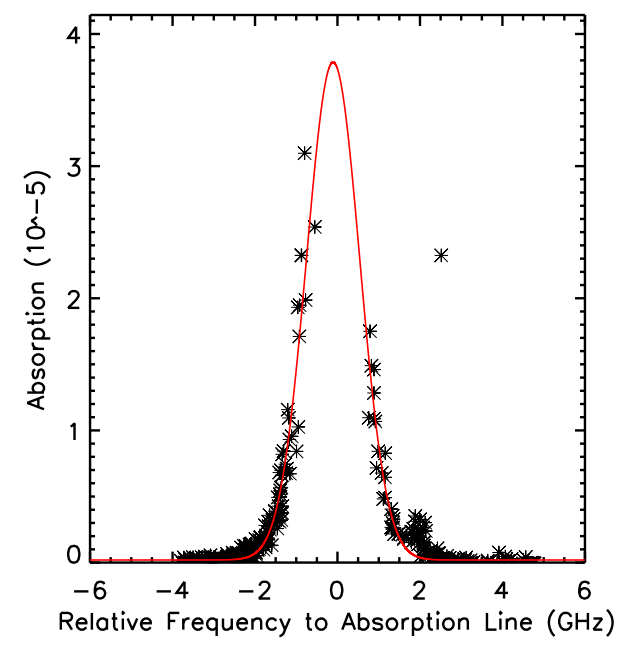

Figure 5.26: (a) Argon neutral CW-CRDS VDF measured at $r f=300 \mathrm{~W}, B=630 \mathrm{G}, P=12.8$ mTorr, mass flow=5 SCCM; T=.08 $\pm .01(\mathrm{eV}), \mathrm{n}=3.0 \pm .3 \times 10^{8}\left(\mathrm{~cm}^{-3}\right)$.

The AR II measurements above (Figures 5.22-5.26) clearly demonstrate that the CW-CRDS diagnostic is capable of measuring argon neutral VDFs at parameters inaccessible to LIF. It is difficult to accurately compare the two diagnostics with so few LIF measurements at the given parameters. The low neutral temperatures are typical of helicon plasma sources. ${ }^{3}$ The argon neutrals are not constrained by the magnetic field, like the ions, and therefore are effectively in thermal equilibrium with the expansion chamber walls.

Figure 5.27 shows the line averaged Ar I temperatures measured using CW-CRDS increased with increasing magnetic field strength. The temperatures calculated using CWCRDS are approximately a factor of two higher than the values obtained using LIF. Figure 5.28 shows that the metastable argon neutral density decreases with increasing magnetic field, which is consistent with an increasing argon ion density as shown in Figure 5.21. 


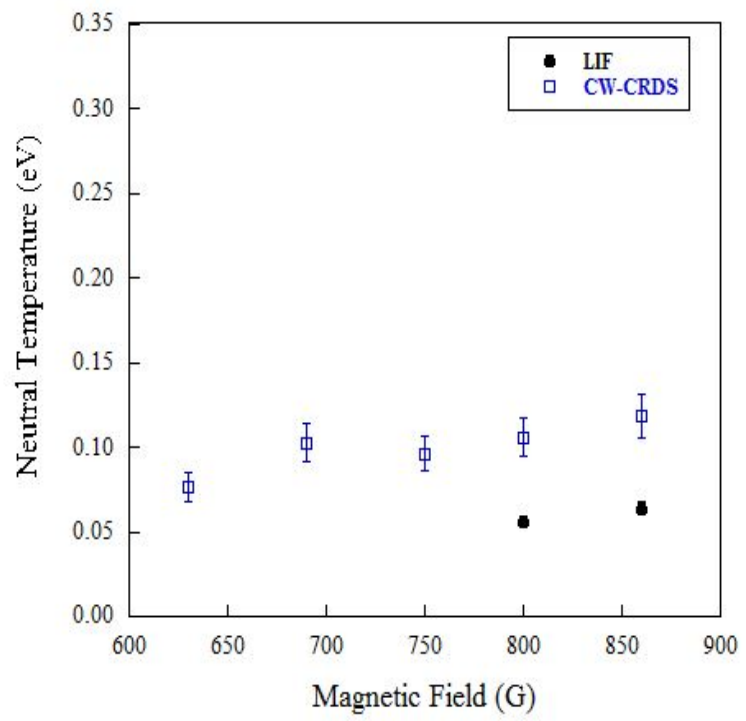

Figure 5.27: Ar I temperatures calculated from LIF and CW-CRDS measurements for different magnetic field strengths.



Figure 5.28: Ar I density calculated with CW-CRDS measurements for different magnetic field strengths. 


\subsection{Discussion}

These measurements demonstrate successful CW-CRDS measurements of argon neutral temperatures and densities for parameters where LIF is insufficiently sensitive. While this is a crucial step in validating the CW-CRDS diagnostic approach, the discrepancies in temperatures measured by CW-CRDS and LIF are of concern.

LIF is spatially localized while CW-CRDS measurements are inherently line integrated. Any temperatures or densities calculated from CW-CRDS measurements are the average value over the spatial path through the plasma. To quantify this effect, spatially resolved LIF measurements could be made along the CW-CRDS optical cavity, summed and compared to the temperature measured using CW-CRDS. Large gradients in temperature and flows along the optical cavity would cause discrepancies between CW-CRDS and LIF measurements.

Some of the CW-CRDS measurements, particularly at parameters that consisted of the highest measured densities for each section, have few if any, data points near the natural absorption line. This lack of data for the peak region of the distribution can affect the quality of the fit resulting in erroneous values for temperature and densities. The data gap occurs because there are so many absorbers present that the peak intensity of the ring-down never exceeds the threshold voltage on the tracking circuit. If the threshold voltage is lowered to collect data at the natural absorption frequency, the $T E M_{00}$ cannot be preferentially sampled for the rest of the distribution. Thus, an improved diagnostic would require a programmable adaptive threshold level.

At parameters where the entire distribution is measured with CW-CRDS, the peak of the distribution tends to be excessively noisy compared to the rest of the distribution. This is an indication of laser power saturation and has been seen in other CW-CRDS measure- 
ments. ${ }^{35}$ Saturation occurs when the target state is depopulated by continuous excitation by high intensity laser light. Absorbers near the natural absorption frequency (nearly at rest) effectively see twice the intensity of the laser light resonant in the cavity (compared to the absorption in the wings). Thus, the peaks are the most likely place to find the first indications of saturation. Figure 5.29 shows an extreme case of a narrow frequency scan (where the laser is near the natural absorption frequency for the majority of the measurement) at the same parameters as those in Figure 5.26. In Figure 5.29 the majority of absorbers near the natural absorption frequency have been depleted. In less extreme cases the peak of the distribution may only have a slight dip or appear noisy. Any saturation of the distribution results in an underestimation of the density and an overestimation of the calculated temperature. If we refer back to Figures 5.20 and 5.21, the largest discrepancy between the temperatures measured by the two diagnostics is for the lowest density condition, where saturation occurs most easily.

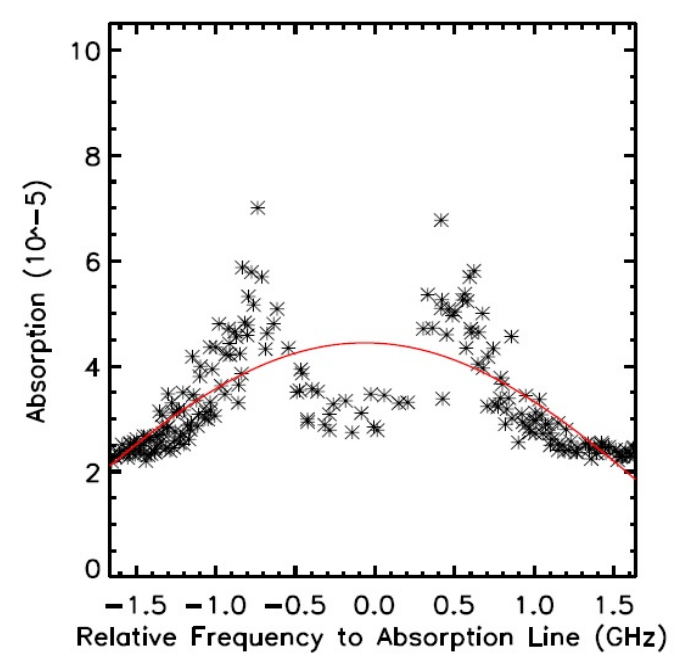

Figure 5.29: Argon neutral CW-CRDS saturation shown at 300 Watts and 630 G. 


\section{Chapter 6}

\section{Conclusion}

The objective of this dissertation was to demonstrate that a cavity ring-down spectroscopy diagnostic is capable of accurately measuring the velocity distribution of plasma species for which LIF is either impossible or has a limited measurement range; that such a system could be constructed in a way so as to avoid degradation by plasma exposure; and that such a system could provide measurements of velocity distributions on reasonable time scales (minutes instead of many hours). The measurements presented here confirm that the upgraded continuous wave cavity ring-down spectroscopy (CW-CRDS) diagnostic provides velocity distribution measurements of the argon ion metastable population with a transition at $668.6138 \mathrm{~nm}$ that are consistent with the standard LIF velocity distribution function measurement of the same transition wavelength. The CW-CRDS diagnostic was then tested on the more problematic argon neutral transition at $667.9125 \mathrm{~nm}$. It is problematic because in LIF measurements, the neutral LIF measurement is extremely sensitive to the electronimpact excitation rate into the lower level of the transition because the initial state of the neutral is not metastable. The CW-CRDS diagnostic provided high quality neutral velocity distribution measurements over a much wider plasma source operating range than the LIF 
method.

Surprisingly, the CW-CRDS approach was most effective when the experimental hardware was greatly simplified. The diode laser beam was shaped to match the Gaussian nature of the $T E M_{00}$ mode in the cavity with a simple single-mode optical fiber. Injection of the probe beam into the cavity was accomplished with a simple two-lens mode-matching system and intrinsic vibrations of the plasma source were used to bring the cavity into resonance with the laser across the entire laser wavelength scan range. Future improvements to the CRDS system are readily identifiable. A variable amplitude threshold circuit is clearly needed to be able to isolate the ring-down measurements near the center of the absorption line because of the dramatic decrease in resonance amplitude near the resonance; a by-product of the improved, over LIF, sensitivity of CW-CRDS. Saturation of the absorption line near the peak of the distribution could be reduced by an improved high-gain, high-bandwidth photodetector. The current system trades off bandwith with gain and at the highest gain settings, the detector significantly limited the bandwidth of the ring down time measurements. With an improved detector, lower injected laser power levels would still yield reasonable signal-tonoise levels.

Additional validation of the CW-CRDS velocity distribution measurements will require spatially resolved LIF measurements along the CW-CRDS optical cavity to measure the temperature and flow profiles that might be modifying the measured width of the velocity distributions. The measurements presented here are a vast improvement over the line widths obtained with the first version of the CW-CRDS diagnostic and, for the most part, are consistent with the LIF measurements. One tremendous advantage of the CW-CRDS technique is the ability to measure the absolute density of the absorbing species without need of any calibration. These measurements are ideally suited for validating collisional radiative models 
of plasma sources and such comparisons are yet to be made in the literature.

Perhaps the most important implication of this work is that the CW-CRDS diagnostic approach developed for these experiments can, with the appropriate light source and mirrors, be utilized for any plasma species with a natural absorption line. The validation of the CWCRDS technique described in this work opens up all manner of plasma sources and species for compact, laser-based, velocity distribution function measurements. Plasma species without readily accessible LIF schemes have defied precise velocity distribution measurements for years. This CW-CRDS diagnostic will work for any plasma species with an absorption line. 


\section{References}

[1] R. Murphy, I. Biloiu, and E. E. Scime Poster session presented at: 4 th Annual Meeting of the APS Division of Plasma Physics, 2005; Denver .CO.

[2] A. M. Keesee. PhD thesis, West Virginia Univesity, 2006.

[3] A. M. Keesee, E. E. Scime, and R. F. Boivin Rev. Sci. Instrum., vol. 75, 2004.

[4] D. Z. Anderson, J. C. Frish, and C. S. Masser Appl. Optics Lett., vol. 23, 1984.

[5] A. O’Keege and D. A. G. Deacon Rev. Sci. Instrum., vol. 59, 1988.

[6] J. T. Hodges, J. P. Looney, and R. D. van Zee Appl. Optics., vol. 35, 1996.

[7] J. T. Hodges, J. P. Looney, and R. D. van Zee J. Chem. Phys., vol. 105, 1996.

[8] D. Romanini, N. S. A. A. Kachanov, and R. Stoeckel Chem. Phys. Lett., vol. 264, 1997.

[9] B. C. Lee, L. T. W. Huang, N. Yamamoto, A. D. Gallimore, and A. P. Yalin Rev. Sci. Instrum., vol. 85, 2014.

[10] N. Srivastava and C. Wang Journal of Applied Physics, vol. 110, 2011.

[11] P. Sahay, S. T. Scherrer, and C. Wang Rev, vol. 83, 2012.

[12] S. C. Thakur, D. McCarren, J. Jerry Carr, and E. E. Scime Rev. Sci. Instrum., vol. 83, 2012.

[13] R. Bowers, C. Legendy, and F. E. Rose Phys. Rev. Lett., vol. 7, p. 339, 1961.

[14] G. N. Harding and P. C. Thonemann Proc. Phys. Soc., no. 85, p. 317, 1965. 
[15] R. W. Boswell Plasma Physics and Controlled Fusion, vol. 26, pp. 1147-1162, 1984.

[16] F. F. Chen vol. 33, pp. 339-364, 1991.

[17] J. L. Kline. PhD thesis, West Virginia University, 2002.

[18] C. Biloiu, E. E. Scime, I. A. Biloiu, and X. Sun Journal of Applied Physics, vol. 102, p. $053303,2007$.

[19] S. C. Thakur. PhD thesis, West Virginia University, 2010.

[20] D. J. Lewis Master's thesis, West Virginia University, 2008.

[21] M. E. Galante. PhD thesis, West Virginia University, 2013.

[22] F. F. Chen tech. rep., UCLA Electrical Engineering Dept. and Institute of Plasma And Fusion Research, 1992.

[23] R. Hardin. PhD thesis, West Virginia University, 2008.

[24] Y. T. Yardley and C. B. Moore Journal of Chemical Physics, vol. 45, p. 1066, 1966.

[25] R. A. Stern and J. A. Johnson Phys. Rev. Lett., vol. 34, p. 1548, 1975.

[26] D. N. Hill, S. Fornaca, and M. G. Wikham Rev. Sci. Instrum., vol. 54, p. 309, 1983.

[27] R. F. Boivin tech. rep., West Virginia University, 1999.

[28] E. E. Scime, P. A. Keiter, M. W. Zintil, M. M. Balkey, J. L. Kline, and M. E. Koepke Plasma Sources Sci. Technol., vol. 7, pp. 186-191, 1998.

[29] G. Berden and R. Engeln. Wiley, 2009.

[30] H. Kogelnik and T. Li Applied Optics, vol. 5, no. 10, 1966. 
[31] M. Mazurenka, A. j. Orr-Ewing, R. Peverall, and G. A. D. Ritchie Chem. Phys. Lett., vol. $101,2005$.

[32] M. M. Hemerik. PhD thesis, Universiteitsdrukkerij Technische Universiteit Eindhoven, 2001.

[33] W. Huang, A. D. Gallimore, T. B. Smith, and A. P. Yalin Presented at the 32nd International Electric Propulsion Conference, 2011.

[34] H. Huang and K. K. Lehmann Applied Physics B: Lasers and Optics, vol. 94, pp. 355$366,2009$.

[35] P. Macko, D. Romanini, and N. adeghi Workshop on Frontiers in Low Temperature Plasma Diagnostics IV, 2001. 